\title{
RECTILINEAR VORTEX SHEETS OF INVISCID LIQUID-GAS TWO-PHASE FLOW: LINEAR STABILITY*
}

\author{
LIZHI RUAN ${ }^{\dagger}$, DEHUA WANG ${ }^{\ddagger}$, SHANGKUN WENG ${ }^{\S}$, AND CHANGJIANG ZHU
}

\begin{abstract}
The vortex sheet solutions are considered for the inviscid liquid-gas two-phase flow. In particular, the linear stability of rectilinear vortex sheets in two spatial dimensions is established for both constant and variable coefficients. The linearized problem of vortex sheet solutions with constant coefficients is studied by means of Fourier analysis, normal mode analysis, and Kreiss symmetrizer, while the linear stability with variable coefficients is obtained by Bony-Meyer paradifferential calculus theory. The linear stability is crucial to the existence of vortex sheet solutions of the nonlinear problem. A novel symmetrization and some weighted Sobolev norms are introduced to study the hyperbolic linearized problem with characteristic boundary.
\end{abstract}

Key words. Inviscid liquid-gas two-phase flow, vortex sheet, linear stability.

AMS subject classifications. 34B05, 35L50, 35L65, 76T10.

\section{Introduction}

Two-phase or multi-phase flows are concerned with flows with two or more components and have a wide range of applications in nature, engineering, and biomedicine. Examples include sediment transport, geysers, volcanic eruptions, clouds, rain in natural and climate systems, mixture of oil and natural gas in extraction tubes of oil exploitation, oil transportation, steam generators, cooling systems, mixture of hot water and vapor of water in cooling tubes of nuclear power stations in energy production, bubble columns, aeration systems, tumor biology, anticancer therapies, developmental biology, plant physiology in chemical engineering, medical and genetic engineering, bioengineering, and so on. Multi-phase flow is much more complicated than single-phase flow due to the existence of a moving and deformable interface and its interactions with multi-phases $[6,28,31,32]$. In this paper, we consider the following system of partial differential equations for the compressible inviscid liquid-gas two-phase flow of drift-flux type:

$$
\left\{\begin{array}{l}
\partial_{t} m+\nabla \cdot(m \mathbf{u})=0 \\
\partial_{t} n+\nabla \cdot(n \mathbf{u})=0 \\
\partial_{t}(n \mathbf{u})+\nabla \cdot(n \mathbf{u} \otimes \mathbf{u})+\nabla p(m, n)=0
\end{array}\right.
$$

where $m=\alpha_{g} \rho_{g}$ and $n=\alpha_{l} \rho_{l}$ denote the gas mass and the liquid mass, respectively, $\alpha_{g}, \alpha_{l} \in[0,1]$ denote the gas and liquid volume fractions satisfying $\alpha_{g}+\alpha_{l}=1, \rho_{g}$ and $\rho_{l}$ denote the gas and liquid densities; $\mathbf{u}$ denotes the mixed velocity of the liquid and the gas, and $p$ is the common pressure for both phases. Wang.

*Received: April 1, 2015; accepted (in revised form): June 26, 2015. Communicated by Yaguang

${ }^{\dagger}$ The Hubei Key Laboratory of Mathematical Physics, School of Mathematics and Statistics, Central China Normal University, Wuhan 430079, P.R. China (rlz@mail.ccnu.edu.cn).

${ }^{\ddagger}$ Department of Mathematics, University of Pittsburgh, Pittsburgh, PA 15260, USA (dwang@math. pitt.edu).

§Pohang Mathematics Institute, Pohang University of Science and Technology. Hyoja-Dong San 31, Nam-Gu, Pohang, Gyungbuk, 790-784, Republic of Korea (skwengmath@gmail.com).

ISchool of Mathematics, South China University of Technology, Guangzhou 510641, P.R. China (machjzhu@scut.edu.cn). 
In fact, the model (1.1) is a simplified version of the following general two-phase flow model:

$$
\left\{\begin{array}{l}
\partial_{t}\left(\alpha_{g} \rho_{g}\right)+\nabla \cdot\left(\alpha_{g} \rho_{g} \mathbf{u}_{g}\right)=0 \\
\partial_{t}\left(\alpha_{l} \rho_{l}\right)+\nabla \cdot\left(\alpha_{l} \rho_{l} \mathbf{u}_{l}\right)=0 \\
\partial_{t}\left(\alpha_{g} \rho_{g} \mathbf{u}_{g}+\alpha_{l} \rho_{l} \mathbf{u}_{l}\right)+\nabla \cdot\left(\alpha_{g} \rho_{g} \mathbf{u}_{g} \otimes \mathbf{u}_{g}+\alpha_{l} \rho_{l} \mathbf{u}_{l} \otimes \mathbf{u}_{l}\right)+\nabla p\left(\alpha_{g} \rho_{g}, \alpha_{l} \rho_{l}\right)=0
\end{array}\right.
$$

where $\alpha_{g}, \alpha_{l}$ and $\rho_{g}, \rho_{l}$ denote the same as above and where $\mathbf{u}_{g}$ and $\mathbf{u}_{l}$ denote the gas and liquid velocities, respectively. Just as in $[14,19,23,35,57,58]$, in order to help further understand the behavior of the solution to the general model, we use two densities and only one fluid velocity to study the simplified two-phase flow model, i.e. we restrict ourselves to a flow regime where liquid and gas velocities can be assumed to be equal: $\mathbf{u}_{g}=\mathbf{u}_{l}=\mathbf{u}$. Since the liquid phase is much heavier than the gas phase, we also neglect the gas phase in the mixture momentum equations.

In general, the pressure law $p(m, n)$ should be a nonlinear complicated function of the densities, we will follow the simplification made in [18] which guarantees that the model is thermo-mechanically consistent in the sense that one can get a basic energy estimate easily. We assume that the pressure $p$ is a smooth function of $(m, n)$ defined on $(0,+\infty) \times(0,+\infty)$, and in particular we take the pressure of the following form (see e.g. [18]):

$$
p(m, n)=\left(c_{1} m+c_{2} n\right)^{2} P^{\prime}\left(c_{1} m+c_{2} n\right),
$$

where $c_{1}, c_{2}$ are positive constants and $P=P(\rho)$ is a smooth function such as $P(\rho)=$ $\rho^{\gamma-1}, \gamma>1$. Without loss of generality, we take $c_{1}=c_{2}=1$ and hence the pressure is

$$
p(m, n)=(\gamma-1)(m+n)^{\gamma}
$$

and

$$
p_{m}(m, n)=p_{n}(m, n)=\gamma(\gamma-1)(m+n)^{\gamma-1}
$$

We shall see in the next section that (1.1) is a non-strictly hyperbolic system of conservation laws in the region $(m, n) \in(0,+\infty) \times(0,+\infty)$.

The viscous two-phase flows have been investigated extensively. In particular, the existence, uniqueness, regularity, asymptotic behavior, decay rate estimates, and blowup phenomena of solutions to various one-dimensional and multi-dimensional viscous two-phase flows have been studied recently in $[13,14,18,19,23,24,26,35,53,55-58]$ and related references therein. The theory of the inviscid two-phase flow (1.1) is comparatively mathematically underdeveloped although there have been many numerical studies; see $[3,4,6,9,15-18,21]$ and their references. In this paper, we are concerned with the rectilinear vortex sheet problem for the two-phase flow (1.1) in the two-dimensional space $\mathbb{R}^{2}$. A velocity discontinuity in an inviscid flow is called a vortex sheet, which yields a concentration of vorticity along the discontinuity front (see [11]). In the threedimensional space, a vortex sheet has vorticity concentrated along a surface in the space. In two-dimensional space, the vorticity is concentrated along a curve in the plane.

Vortex sheets occur commonly in nature, sciences, and engineering and have attracted enormous studies. Some early studies on the linear stability of planar and rectilinear compressible vortex sheets can be found in $[20,43]$. In three spatial dimensions, it is known that the planar vortex sheets is unstable (see e.g. [45]). In the two-dimensional 
case, subsonic vortex sheets are also unstable, but the supersonic vortex sheets are linearly stable (see e.g. $[43,45])$. For the incompressible theory of vortex sheets, we refer the readers to $[2,7,29,30,36,37,41,47,49,54]$ and the references therein.

Our work in this paper is inspired by $[8,11,12,44,48]$ on the stability of planar and rectilinear vortex sheets for the compressible isentropic [11,12] and non-isentropic Euler equations [44], and the ideal compressible magnetohydrodynamics (MHD) $[8,48]$. The linear stability of compressible vortex sheets for the isentropic Euler equations in two spatial dimensions was studied under a supersonic condition and an energy estimate for the linearized boundary value problem was proved in [11]. The nonlinear stability was analyzed in [12] based on the linear analysis in [11] and the Nash-Moser iteration. These two papers of Coulombel and Secchi $[11,12]$ are the pioneering works in the direction of compressible vortex sheets. The result on linear stability for the isentropic case in [11] was also extended to the non-isentropic case (see [44]). The linear and nonlinear stability of current-vortex sheets for the ideal compressible MHD was studied in $[8,48,51]$. The vortex sheets in the three-dimensional steady compressible flows were considered in $[50,52]$.

As mentioned in $[8,11,12,44,48]$, the existence of compressible vortex sheets is a nonlinear hyperbolic problem with free boundaries. Since the vortex sheet is a contact discontinuity, the free boundary is characteristic. Thus, we have a hyperbolic initialboundary value problem with a characteristic boundary, violating the uniform KreissLopatinskii condition and causing loss of derivatives with respect to the source terms for energy estimates as well as loss of control of the tangential velocity (the "characteristic part" of the solution) on the boundary. Thanks to the ellipticity of the boundary conditions for the unknown front, we will be able to gain one derivative as for shock waves in Majda [39].

The purpose of this paper is to establish linear stability with both constant and variable coefficients of rectilinear vortex sheets for the two-phase flow (1.1) in two spatial dimensions. We remark that our paper is strongly motivated by the ideas of the pioneering paper [11] and generalizes the work of [11] to the two-phase flow. To this end, we organize the analysis as follows. In Section 2, we first set up the vortex sheet problem as a free-boundary problem by analyzing the Rankine-Hugoniot conditions corresponding to the vortex sheets of equation (1.1), and then we reformulate this problem into a fixed-boundary problem by employing the standard partial hodograph transformation $[8,11,48]$ and tedious calculations. We note that a natural approach of introducing the Lagrangian coordinates to fix the boundary does not seem to work for the vortex sheet problem.

In Section 3, in order to obtain the a priori energy estimates mentioned above, we first in Subsection 3.1 introduce a "good" symmetric form of the linearized version of the liquid-gas two-phase flow system (1.1), which plays a crucial role in our analysis. To the best of our knowledge this "good" symmetric form is new and does not follow directly from any known symmetrization. As emphasized in [8,11,48], a "good" symmetric form is very important, although it is easy to perform a "trivial" symmetrization of system (1.1). However, a "good" symmetric form is required to separate the "characteristic part" and "non-characteristic part" of solutions so that one can get rid of the singularity from the boundary matrix and reduce the symbolic characteristic case to the noncharacteristic case. Fortunately, we find a "good" transformation (see (3.5), (3.6)) which leads to a "good" symmetric form of the linearized equations. We remark, as pointed out in [25], that most hyperbolic operators are not symmetrizable in $d(d \geq$ 2) spatial dimensions although the physical systems of compressible Euler equations, 
MHD, and liquid-gas two-phase flows can be reduced to symmetric hyperbolic systems of conservation laws in time-space dimension $d+1$. Then, in Subsection 3.2, some weighted Sobolev norms are introduced since there is a loss of control on derivatives in the normal direction for the hyperbolic problem with characteristic boundary. The main result on the linear stability for constant coefficients is stated.

In Section 4, we shall prove the main theorem on the linearized problem with constant coefficients by the normal mode analysis of the linearized problem and constructing a degenerate Kreiss symmetrizers in order to derive our energy estimate. As in $[11,39,40]$, the linearized Rankine-Hugoniot conditions form an elliptic system for the unknown front, which is very important when eliminating the unknown front and considering a standard-boundary value problem with a symbolic boundary condition. Based on our new observation and the property that no jump occurs in the sum of mass of both liquid and gas even though each has jump individually, we introduce an appropriate $C^{\infty}$ smooth mapping $Q$ to obtain the elliptic estimate on the corresponding symbol and formally cast our problem in the framework of [44], which allows us directly employ their construction of symmetrizers to simplify our calculation and mathematical analysis. However, the construction of the symmetrizers is microlocal. Near the neighborhood of the poly point, the construction of symmetrizers is different from those in [11] and [44] for the original symbolic algebraic-differential equations.

In Section 5, we discuss and formulate the linearized problem of vortex sheets with variable coefficients and state the main result on the linear stability. In Section 6, we shall prove the main theorem on the linearized problem with variable coefficients by paralinearized techniques and Bony-Meyer paradifferential calculus theory $[5,42]$. The key point is to freeze the coefficients to turn the variable coefficient problem into the constant coefficient problem. The critical set of Lopatinski determinants will be constructed in detail. This is a key point of microlocal analysis in the neighborhood of bicharacteristic curve along which singularities propagate. In the analysis, we need some precise calculations, which will be collected in Appendix A.

We conclude the introduction by remarking that the linear stability studied in this paper is a crucial step towards the local-in-time existence of vortex sheet solutions of the nonlinear problem. This nonlinear stability will be investigated in a forthcoming paper based on the linear stability obtained in this paper and the Nash-Moser iteration method.

\section{Vortex sheet problem and reformulation}

In this section, we first set up the vortex sheet problem as a free boundary problem and then reformulate it into an initial-boundary value problem with fixed boundaries.

2.1. Vortex sheet problem. We will consider the liquid-gas two-phase flow (1.1) in the whole space $\mathbb{R}^{2}$ and present the analysis which leads to a vortex sheet problem. Let $x=\left(x_{1}, x_{2}\right)$ be the space variable in $\mathbb{R}^{2}$, and let $v$ and $u$ be the first and second components, respectively, of the velocity field. Thus $\mathbf{u}=(v, u) \in \mathbb{R}^{2}$. Then, for

$$
U=(m, n, \mathbf{u})^{\top} \in(0,+\infty) \times(0,+\infty) \times \mathbb{R}^{2},
$$

we define the following matrices:

$$
A_{1}(U)=\left(\begin{array}{cccc}
v & 0 & m & 0 \\
0 & v & n & 0 \\
\frac{p_{m}}{n} & \frac{p_{n}}{n} & v & 0 \\
0 & 0 & 0 & v
\end{array}\right), \quad A_{2}(U)=\left(\begin{array}{llll}
u & 0 & 0 & m \\
0 & u & 0 & n \\
0 & 0 & u & 0 \\
\frac{p_{m}}{n} & \frac{p_{n}}{n} & 0 & u
\end{array}\right) .
$$


Denote the spatial partial derivatives by

$$
\partial_{1}=\partial_{x_{1}}, \quad \partial_{2}=\partial_{x_{2}}
$$

In the region where $(m, n, \mathbf{u})$ is smooth (i.e. differentiable), (1.1) is equivalent to the following quasilinear form:

$$
\partial_{t} U+A_{1}(U) \partial_{1} U+A_{2}(U) \partial_{2} U=0
$$

The eigenvalues of the matrix

$$
A(U, \xi)=A(m, n, \mathbf{u}, \xi)=\xi_{1} A_{1}(U)+\xi_{2} A_{2}(U), \quad \forall \xi=\left(\xi_{1}, \xi_{2}\right) \in \mathbb{R}^{2},
$$

are given by

$$
\left\{\begin{array}{l}
\lambda_{1}(U, \xi)=\xi \cdot \mathbf{u}-|\xi| \sqrt{\frac{m p_{m}+n p_{n}}{n}} \quad \text { with multiplicity } m_{1}=1 \\
\lambda_{2}(U, \xi)=\xi \cdot \mathbf{u} \quad \text { with multiplicity } m_{2}=2 \\
\lambda_{3}(U, \xi)=\xi \cdot \mathbf{u}+|\xi| \sqrt{\frac{m p_{m}+n p_{n}}{n}} \quad \text { with multiplicity } m_{3}=1
\end{array}\right.
$$

in the region $U \in(0,+\infty) \times(0,+\infty) \times \mathbb{R}^{2}$. The eigenvector corresponding to the second eigenvalue field $\lambda_{2}(U, \xi)$ is given by

$$
r_{2}(U, \xi)=\left(\xi_{2},-\xi_{1}, \xi_{2} p_{n},-\xi_{1} p_{m}\right)^{\top}
$$

If $p_{n}=p_{m}$ for the pressure $p$, the second characteristic field of system (2.2) (or (1.1)) is linearly degenerate, which leads us to consider the vortex sheet (contact discontinuity) solutions for the two-phase flow. In fact, a vortex sheet (contact discontinuity) solution is a weak solution with possible strong discontinuities.

Definition 2.1 (Weak solution). Let $(m, n, \boldsymbol{u})$ be a smooth function of $\left(t, x_{1}, x_{2}\right)$ on either side of a smooth surface $\Gamma:=\left\{x_{2}=\varphi\left(t, x_{1}\right), t>0, x_{1} \in \mathbb{R}\right\}$. Then, $(m, n, \boldsymbol{u})$ is a weak solution of (1.1) if and only if $(m, n, \boldsymbol{u})$ is a classical solution of (1.1) on both sides of $\Gamma$ and the Rankine-Hugoniot conditions hold at each point of $\Gamma$ :

$$
\left\{\begin{array}{l}
\partial_{t} \varphi[m]-[m \boldsymbol{u} \cdot \nu]=0 \\
\partial_{t} \varphi[n]-[n \boldsymbol{u} \cdot \nu]=0 \\
\partial_{t} \varphi[n \boldsymbol{u}]-[(n \boldsymbol{u} \cdot \nu) \boldsymbol{u}]-[p] \nu=0
\end{array}\right.
$$

where $\nu:=\left(-\partial_{1} \varphi, 1\right)$ is a space normal vector to $\Gamma$ and $\left(-\partial_{t} \varphi,-\partial_{1} \varphi, 1\right)=\left(-\partial_{t} \varphi, \nu\right)$ is a time-space conormal vector to $\Gamma$. As usual, $[q]=q^{+}-q^{-}$denotes the jump of a quantity $q$ across the interface $\Gamma$.

Moreover, vortex sheets have continuous normal velocity and possible jump of tangential velocity, yielding a concentration of vorticity along the discontinuity front. The velocity of the front $\partial_{t} \varphi$ satisfies $\partial_{t} \varphi=\mathbf{u}^{+} \cdot \nu=\mathbf{u}^{-} \cdot \nu$, which means that the first two equations in (2.4) are automatically satisfied and the third one gives $p^{+}=p^{-}$. We are now in a position to give the rigorous definition of a vortex sheet solution (contact discontinuity in sense of Lax [33]) of the liquid-gas two-phase flow.

Definition 2.2 (Vortex sheet solution). A piecewise smooth vector-function $(m, n, \boldsymbol{u})$ is called a rectilinear vortex sheet solution of $(1.1)$ if $(m, n, \boldsymbol{u})$ is a classical 
solution of (1.1) on either side of the smooth surface $\Gamma$ and the Rankine-Hugoniot conditions (2.4) are satisfied at each point of $\Gamma$ in the following way:

$$
\partial_{t} \varphi=\boldsymbol{u}^{+} \cdot \nu=\boldsymbol{u}^{-} \cdot \nu, \quad p^{+}=p^{-}
$$

We note that (2.5) implies

$$
\partial_{t} \varphi=\mathbf{u}^{+} \cdot \nu=\mathbf{u}^{-} \cdot \nu, \quad m^{+}+n^{+}=m^{-}+n^{-} .
$$

Then the problem of existence and stability of vortex sheets solution can be formulated as the following free boundary problem: determine

$$
U^{ \pm}\left(t, x_{1}, x_{2}\right)=\left(m^{ \pm}, n^{ \pm}, v^{ \pm}, u^{ \pm}\right)\left(t, x_{1}, x_{2}\right) \in(0,+\infty) \times(0,+\infty) \times \mathbb{R}^{2}
$$

and a free boundary $\Gamma:=\left\{x_{2}=\varphi\left(t, x_{1}\right), t>0, x_{1} \in \mathbb{R}\right\}$ such that

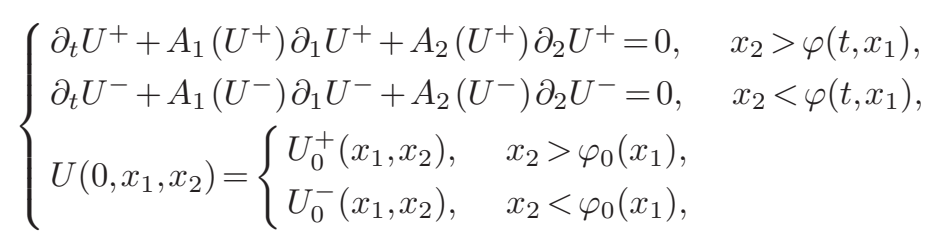

satisfying the jump conditions on $\Gamma$ :

$$
\partial_{t} \varphi=-v^{+} \partial_{1} \varphi+u^{+}=-v^{-} \partial_{1} \varphi+u^{-}, \quad m^{+}+n^{+}=m^{-}+n^{-},
$$

where $\varphi_{0}\left(x_{1}\right)=\varphi\left(0, x_{1}\right)$.

Note that the first two equalities in (2.8) are just the eikonal equations

$$
\varphi_{t}+\lambda_{2}\left(m^{+}, n^{+}, \mathbf{u}^{+}, \partial_{1} \varphi\right)=0 \quad \text { and } \varphi_{t}+\lambda_{2}\left(m^{-}, n^{-}, \mathbf{u}^{-}, \partial_{1} \varphi\right)=0,
$$

on $\left\{x_{2}=0\right\}$, where the eigenvalue $\lambda_{2}(m, n, \mathbf{u}, \xi)$ is defined in (2.3). To prove the existence of tangential discontinuities (vortex sheets) for the free-boundary problem (2.7) and (2.8), one needs to find a solution $(U, \varphi)\left(t, x_{1}, x_{2}\right)$ of the problem $(2.7)$ and $(2.8)$ at least locally in time. More precisely, we need to prove the local-in-time well-posedness of the problem (2.7) and (2.8). Our goal in this paper is to establish the well-posedness of the linearized problem resulting from the linearization of (2.7) and (2.8) around a background vortex sheet (piecewise constant) solution. As discussed in [11], for the isentropic Euler equations (1.1), these solutions are exactly the contact discontinuities in the sense of Lax [33].

2.2. Reformulation. We now reformulate the free-boundary problem into a fixed-boundary problem. To straighten the unknown front, we employ the standard partial hodograph transformation (see e.g. $[8,11,48]$ ):

$$
t=\tilde{t}, \quad x_{1}=\tilde{x}_{1}, \quad x_{2}=\Phi^{ \pm}\left(\tilde{t}, \tilde{x}_{1}, \tilde{x}_{2}\right)
$$

with some smooth functions $\Phi^{ \pm}$satisfying

$$
\begin{aligned}
& \pm \partial_{\tilde{x}_{2}} \Phi^{ \pm}\left(\tilde{t}, \tilde{x}_{1}, \tilde{x}_{2}\right) \geq \kappa>0 \\
& \Phi^{+}\left(\tilde{t}, \tilde{x}_{1}, 0\right)=\Phi^{-}\left(\tilde{t}, \tilde{x}_{1}, 0\right)=\varphi\left(\tilde{t}, \tilde{x}_{1}\right),
\end{aligned}
$$

for some constant $\kappa>0$. Under (2.10), the domains are transformed into $\left\{\tilde{x}_{2}>0\right\}$ and the free boundary $\Gamma$ into the fixed boundary $\left\{\tilde{x}_{2}=0\right\}$. More precisely, the unknowns 
$\left(m^{ \pm}, n^{ \pm}, \mathbf{u}^{ \pm}\right)\left(t, x_{1}, x_{2}\right)$, that are smooth on either side of $\left\{x_{2}=\varphi\left(t, x_{1}\right)\right\}$, are replaced by the functions

$$
\begin{aligned}
\left(m_{\sharp}^{ \pm}, n_{\sharp}^{ \pm}, \mathbf{u}_{\sharp}^{ \pm}\right)\left(\tilde{t}, \tilde{x}_{1}, \tilde{x}_{2}\right) & =\left(m^{ \pm}, n^{ \pm}, \mathbf{u}^{ \pm}\right)\left(t, x_{1}, x_{2}\right) \\
& =\left(m^{ \pm}, n^{ \pm}, \mathbf{u}^{ \pm}\right)\left(\tilde{t}, \tilde{x}_{1}, \Phi^{ \pm}\left(\tilde{t}, \tilde{x}_{1}, \tilde{x}_{2}\right)\right),
\end{aligned}
$$

which are smooth on the fixed domain $\left\{\tilde{x}_{2}>0\right\}$.

From now on we drop the tildes for simplicity of notation. Let us denote by $v_{\sharp}$ and $u_{\sharp}$ the two components of the velocity, that is, $\mathbf{u}_{\sharp}^{ \pm}=\left(v_{\sharp}^{ \pm}, u_{\sharp}^{ \pm}\right)$. Then, the smooth solutions $\left(m_{\sharp}^{ \pm}, n_{\sharp}^{ \pm}, v_{\sharp}^{ \pm}, u_{\sharp}^{ \pm}, \Phi^{ \pm}\right)$satisfy the following initial-boundary value problem with the fixed boundary $x_{2}=0$ :

$$
\begin{aligned}
& \partial_{t} m_{\sharp}^{ \pm}+v_{\sharp}^{ \pm} \partial_{1} m_{\sharp}^{ \pm}+\left(u_{\sharp}^{ \pm}-\partial_{t} \Phi^{ \pm}-v_{\sharp}^{ \pm} \partial_{1} \Phi^{ \pm}\right) \frac{\partial_{2} m_{\sharp}^{ \pm}}{\partial_{2} \Phi^{ \pm}} \\
& +m_{\sharp}^{ \pm} \partial_{1} v_{\sharp}^{ \pm}+m_{\sharp}^{ \pm} \frac{\partial_{2} u_{\sharp}^{ \pm}}{\partial_{2} \Phi^{ \pm}}-m_{\sharp}^{ \pm} \frac{\partial_{1} \Phi^{ \pm}}{\partial_{2} \Phi^{ \pm}} \partial_{2} v_{\sharp}^{ \pm}=0, \\
& \partial_{t} n_{\sharp}^{ \pm}+v_{\sharp}^{ \pm} \partial_{1} n_{\sharp}^{ \pm}+\left(u_{\sharp}^{ \pm}-\partial_{t} \Phi^{ \pm}-v_{\sharp}^{ \pm} \partial_{1} \Phi^{ \pm}\right) \frac{\partial_{2} n_{\sharp}^{ \pm}}{\partial_{2} \Phi^{ \pm}} \\
& +n_{\sharp}^{ \pm} \partial_{1} v_{\sharp}^{ \pm}+n_{\sharp}^{ \pm} \frac{\partial_{2} u_{\sharp}^{ \pm}}{\partial_{2} \Phi^{ \pm}}-n_{\sharp}^{ \pm} \frac{\partial_{1} \Phi^{ \pm}}{\partial_{2} \Phi^{ \pm}} \partial_{2} v_{\sharp}^{ \pm}=0, \\
& \partial_{t} v_{\sharp}^{ \pm}+v_{\sharp}^{ \pm} \partial_{1} v_{\sharp}^{ \pm}+\left(u_{\sharp}^{ \pm}-\partial_{t} \Phi^{ \pm}-v_{\sharp}^{ \pm} \partial_{1} \Phi^{ \pm}\right) \frac{\partial_{2} v_{\sharp}^{ \pm}}{\partial_{2} \Phi^{ \pm}} \\
& +\frac{p_{m_{\sharp}^{ \pm}}}{n_{\sharp}^{ \pm}} \partial_{1} m_{\sharp}^{ \pm}-\frac{p_{m_{\sharp}^{ \pm}}}{n_{\sharp}^{ \pm}} \frac{\partial_{1} \Phi^{ \pm}}{\partial_{2} \Phi^{ \pm}} \partial_{2} m_{\sharp}^{ \pm}+\frac{p_{n_{\sharp}^{ \pm}}}{n_{\sharp}^{ \pm}} \partial_{1} n_{\sharp}^{ \pm}-\frac{p_{n_{\sharp}^{ \pm}}}{n_{\sharp}^{ \pm}} \frac{\partial_{1} \Phi^{ \pm}}{\partial_{2} \Phi^{ \pm}} \partial_{2} n_{\sharp}^{ \pm}=0, \\
& \partial_{t} u_{\sharp}^{ \pm}+v_{\sharp}^{ \pm} \partial_{1} u_{\sharp}^{ \pm}+\left(u_{\sharp}^{ \pm}-\partial_{t} \Phi^{ \pm}-v_{\sharp}^{ \pm} \partial_{1} \Phi^{ \pm}\right) \frac{\partial_{2} u_{\sharp}^{ \pm}}{\partial_{2} \Phi^{ \pm}}+\frac{p_{m_{\sharp}^{ \pm}}}{n_{\sharp}^{ \pm}} \frac{\partial_{2} m_{\sharp}^{ \pm}}{\partial_{2} \Phi^{ \pm}}+\frac{p_{n_{\sharp}^{ \pm}}}{n_{\sharp}^{ \pm}} \frac{\partial_{2} n_{\sharp}^{ \pm}}{\partial_{2} \Phi^{ \pm}}=0,
\end{aligned}
$$

in the fixed domain $\left\{x_{2}>0\right\}$, together with the boundary conditions from (2.12) and (2.11):

$$
\begin{aligned}
& \left.\Phi^{+}\left(t, x_{1}, x_{2}\right)\right|_{x_{2}=0}=\left.\Phi^{-}\left(t, x_{1}, x_{2}\right)\right|_{x_{2}=0}=\varphi\left(t, x_{1}\right), \\
& \left.\left(v_{\sharp}^{+}-v_{\sharp}^{-}\right)\left(t, x_{1}, x_{2}\right)\right|_{x_{2}=0} \partial_{1} \varphi\left(t, x_{1}\right)-\left.\left(u_{\sharp}^{+}-u_{\sharp}^{-}\right)\left(t, x_{1}, x_{2}\right)\right|_{x_{2}=0}=0, \\
& \partial_{t} \varphi\left(t, x_{1}\right)+\left.v_{\sharp}^{+}\left(t, x_{1}, x_{2}\right)\right|_{x_{2}=0} \partial_{1} \varphi\left(t, x_{1}\right)-\left.u_{\sharp}^{+}\left(t, x_{1}, x_{2}\right)\right|_{x_{2}=0}=0, \\
& \left.\left(m_{\sharp}^{+}+n_{\sharp}^{+}\right)\left(t, x_{1}, x_{2}\right)\right|_{x_{2}=0}-\left.\left(m_{\sharp}^{-}+n_{\sharp}^{-}\right)\left(t, x_{1}, x_{2}\right)\right|_{x_{2}=0}=0 .
\end{aligned}
$$

For contact discontinuities, one can choose the change of variables $\Phi^{ \pm}$satisfying the eikonal equations:

$$
\partial_{t} \Phi^{ \pm}+\lambda_{2}\left(m_{\sharp}^{ \pm}, n_{\sharp}^{ \pm}, \mathbf{u}_{\sharp}^{ \pm}, \partial_{1} \Phi^{ \pm}\right)=\partial_{t} \Phi^{ \pm}+v_{\sharp}^{ \pm} \partial_{1} \Phi^{ \pm}-u_{\sharp}^{ \pm}=0,
$$


in the whole closed half-space $\left\{x_{2} \geq 0\right\}$. We know from (2.17) that (2.18) is satisfied on the boundary $\left\{x_{2}=0\right\}$.

Again for the sake of simplicity of notation, we shall drop the symbol $\sharp$ in (2.13)(2.16) and denote $U:=(m, n, v, u)^{\top}$. Then the nonlinear equations (2.13)-(2.16) read

$$
\begin{aligned}
& \partial_{t} U^{+}+A_{1}\left(U^{+}\right) \partial_{1} U^{+}+\frac{1}{\partial_{2} \Phi^{+}}\left(A_{2}\left(U^{+}\right)-\partial_{t} \Phi^{+} I_{4 \times 4}-\partial_{1} \Phi^{+} A_{1}\left(U^{+}\right)\right) \partial_{2} U^{+}=0, \\
& \partial_{t} U^{-}+A_{1}\left(U^{-}\right) \partial_{1} U^{-}+\frac{1}{\partial_{2} \Phi^{-}}\left(A_{2}\left(U^{-}\right)-\partial_{t} \Phi^{-} I_{4 \times 4}-\partial_{1} \Phi^{-} A_{1}\left(U^{-}\right)\right) \partial_{2} U^{-}=0 .
\end{aligned}
$$

Here $A_{1}(U), A_{2}(U)$ are defined by $(2.1)$ and $I_{4 \times 4}$ is the $4 \times 4$ identity matrix. Define the differential operator $L$ as the left side of (2.19). Then system (2.19) becomes

$$
L\left(U^{+}, \nabla \Phi^{+}\right) U^{+}=0, \quad L\left(U^{-}, \nabla \Phi^{-}\right) U^{-}=0,
$$

where $\nabla \Phi^{ \pm}=\left(\partial_{t} \Phi^{ \pm}, \partial_{1} \Phi^{ \pm}, \partial_{2} \Phi^{ \pm}\right)$. With a slight abuse of notation, we also write this system as

$$
L(U, \nabla \Phi) U=0,
$$

where $U$ denotes the vector $\left(U^{+}, U^{-}\right)$and $\Phi$ for $\left(\Phi^{+}, \Phi^{-}\right)$. The two equations in $(2.19)$ are decoupled in the interior of the domain, and their coupling is made through the boundary conditions (2.17).

There exist many simple solutions of (2.20), (2.17), and (2.11) corresponding to rectilinear vortex sheets in the original variables. In the new variables, they are piecewise constant solutions of (2.20), (2.17), and (2.11):

$$
U_{r}=\left(\begin{array}{c}
m_{r} \\
n_{r} \\
v_{r} \\
0
\end{array}\right), \quad U_{l}=\left(\begin{array}{c}
m_{l} \\
n_{l} \\
v_{l} \\
0
\end{array}\right), \Phi_{r, l}\left(t, x_{1}, x_{2}\right) \equiv \pm x_{2}, \quad \varphi \equiv 0,
$$

with the relation

$$
\left(m_{r}+n_{r}\right)-\left(m_{l}+n_{l}\right)=0, \quad v_{r}+v_{l}=0 .
$$

We only consider the case $v_{r} \neq 0$, and without loss of generality we assume $v_{r}>0$. We also assume $m_{r}, m_{l}, n_{r}, n_{l}>0$. In the next section, we study the linearized equations around the special background solution defined by (2.22).

\section{Linear stability: constant coefficients}

In this section, we formulate the linearized problem with constant coefficients and state the main result on the linear stability with constant coefficients.

3.1. The linearized equations. Denote by

$$
\dot{U}_{ \pm}:=\left(\dot{m}_{ \pm}, \dot{n}_{ \pm}, \dot{v}_{ \pm}, \dot{u}_{ \pm}\right), \quad \Psi_{ \pm}
$$

the small perturbations of the exact solution given by (2.22), that is,

$$
U^{ \pm}=U_{r, l}+\dot{U}_{ \pm}, \quad \Phi^{ \pm}=\Phi_{r, l}+\Psi_{ \pm} .
$$

Up to second order, the perturbations $\dot{U}_{ \pm}=\left(\dot{m}_{ \pm}, \dot{n}_{ \pm}, \dot{v}_{ \pm}, \dot{u}_{ \pm}\right)$satisfy the following linearized equations

$$
\begin{aligned}
& \partial_{t} \dot{U}_{+}+A_{1}\left(U_{r}\right) \partial_{1} \dot{U}_{+}+A_{2}\left(U_{r}\right) \partial_{2} \dot{U}_{+}=0 \\
& \partial_{t} \dot{U}_{-}+A_{1}\left(U_{l}\right) \partial_{1} \dot{U}_{-}-A_{2}\left(U_{l}\right) \partial_{2} \dot{U}_{-}=0
\end{aligned}
$$


in the domain $\left\{x_{2}>0\right\}$, as well as the linearized Rankine-Hugoniot relations

$$
\begin{aligned}
& \Psi_{+}=\Psi_{-}=\psi, \\
& \left(v_{r}-v_{l}\right) \partial_{1} \psi-\left(\dot{u}_{+}-\dot{u}_{-}\right)=0, \\
& \partial_{t} \psi+v_{r} \partial_{1} \psi-\dot{u}_{+}=0, \\
& \left(\dot{m}_{+}+\dot{n}_{+}\right)-\left(\dot{m}_{-}+\dot{n}_{-}\right)=0,
\end{aligned}
$$

on the boundary $\left\{x_{2}=0\right\}$. Rewrite equations (3.1) and (3.2) as

$$
\left\{\begin{array}{l}
L^{\prime} \dot{U}=0, \quad \text { if } x_{2}>0 \\
B(\dot{U}, \psi)=0, \quad \text { if } x_{2}=0
\end{array}\right.
$$

where $\dot{U}=\left(\dot{U}_{+}, \dot{U}_{-}\right)$, and

$$
\begin{aligned}
& L^{\prime} \dot{U}=\partial_{t}\left(\begin{array}{c}
\dot{U}_{+} \\
\dot{U}_{-}
\end{array}\right)+\left(\begin{array}{lr}
A_{1}\left(U_{r}\right) & 0 \\
0 & A_{2}\left(U_{l}\right)
\end{array}\right) \partial_{1}\left(\begin{array}{c}
\dot{U}_{+} \\
\dot{U}_{-}
\end{array}\right)+\left(\begin{array}{lr}
A_{1}\left(U_{r}\right) & 0 \\
0 & -A_{2}\left(U_{l}\right)
\end{array}\right) \partial_{2}\left(\begin{array}{c}
\dot{U}_{+} \\
\dot{U}_{-}
\end{array}\right), \\
& B(\dot{U}, \psi)=\left(\begin{array}{c}
\left(v_{r}-v_{l}\right) \partial_{1} \psi-\left(\dot{u}_{+}-\dot{u}_{-}\right) \\
\partial_{t} \psi+v_{r} \partial_{1} \psi-\dot{u}_{+} \\
\left(\dot{m}_{+}+\dot{n}_{+}\right)-\left(\dot{m}_{-}+\dot{n}_{-}\right)
\end{array}\right) .
\end{aligned}
$$

We remark that the interior equations do not involve the perturbation $\psi$. Thus, the operator $L^{\prime}$ only acts on $\dot{U}$. Generally energy estimates for the linearized equations depend on the source terms both in the interior domain and on the boundary. We now consider the linear equations

$$
\left\{\begin{array}{l}
L^{\prime} \dot{U}=f=\left(f_{+}, f_{-}\right)^{\top}, \quad \text { if } x_{2}>0, \\
B(\dot{U}, \psi)=g=\left(g_{1}, g_{2}, g_{3}\right)^{\top}, \quad \text { if } x_{2}=0,
\end{array}\right.
$$

and the goal is to establish the estimates of $\dot{U}$ and $\psi$ in terms of $f$ and $g$ in appropriate functional spaces.

To simplify the calculations, we introduce some new unknown functions by performing the linear and invertible changes of variables

$$
\left(\begin{array}{c}
\dot{m}_{+} \\
\dot{n}_{+} \\
\dot{v}_{+} \\
\dot{u}_{+}
\end{array}\right)=\left(\begin{array}{lccr}
2 n_{r} & 0 & -2 m_{r} & 2 m_{r} \\
-2 n_{r} & 0 & -2 n_{r} & 2 n_{r} \\
0 & 1 & 0 & 0 \\
0 & 0 & 2 c_{r} & 2 c_{r}
\end{array}\right)\left(\begin{array}{l}
W_{1} \\
W_{2} \\
W_{3} \\
W_{4}
\end{array}\right)
$$

and

$$
\left(\begin{array}{l}
\dot{m}_{-} \\
\dot{n}_{-} \\
\dot{v}_{-} \\
\dot{u}_{-}
\end{array}\right)=\left(\begin{array}{llcr}
2 n_{l} & 0 & -2 m_{l} & 2 m_{l} \\
-2 n_{l} & 0 & -2 n_{l} & 2 n_{l} \\
0 & 1 & 0 & 0 \\
0 & 0 & 2 c_{l} & 2 c_{l}
\end{array}\right)\left(\begin{array}{l}
W_{5} \\
W_{6} \\
W_{7} \\
W_{8}
\end{array}\right)
$$

where $c_{r, l}$ are defined by

$$
c_{r, l}=\sqrt{\left(1+\frac{m_{r, l}}{n_{r, l}}\right) p_{n}}
$$


and $p_{n}=p_{n}\left(m_{r}, n_{r}\right)=p_{n}\left(m_{l}, n_{l}\right)$ are given in (1.3). We also define the following vectors:

$$
\begin{aligned}
& W:=\left(W_{1}, W_{2}, W_{3}, W_{4}, W_{5}, W_{6}, W_{7}, W_{8}\right)^{\top}, \\
& W^{c}:=\left(W_{1}, W_{2}, W_{5}, W_{6}\right)^{\top} \\
& W^{n c}:=\left(W_{3}, W_{4}, W_{7}, W_{8}\right)^{\top} .
\end{aligned}
$$

The notation $W^{c}$ and $W^{n c}$ are introduced in order to separate the "characteristic part" of the vector $W$ and the "non-characteristic part" of $W$. It is obvious that estimating $W$ is equivalent to estimating $\dot{U}$. The vector $W$ satisfies

$$
\left\{\begin{array}{l}
\overline{\mathcal{L}} W:=\partial_{t} W+\overline{\mathcal{A}}_{1} \partial_{1} W+\overline{\mathcal{A}}_{2} \partial_{2} W=\bar{f}, \quad \text { if } x_{2}>0 \\
\overline{\mathcal{B}}\left(W^{n c}, \psi\right):=\left.\underline{M} W^{n c}\right|_{x_{2}=0}+\underline{b}\left(\begin{array}{c}
\partial_{t} \psi \\
\partial_{1} \psi
\end{array}\right)=\bar{g}, \quad \text { if } x_{2}=0
\end{array}\right.
$$

with new $\bar{f}$ and $\bar{g}$ and

$$
\begin{aligned}
& \overline{\mathcal{A}}_{1}:=\left(\begin{array}{cccrrrrr}
v_{r} & 0 & 0 & 0 & & & \\
0 & v_{r} & -2 c_{r}^{2} & 2 c_{r}^{2} & \multicolumn{2}{c}{\mathbf{O}} & \\
0 & -\frac{1}{4} & v_{r} & 0 & & & \\
0 & \frac{1}{4} & 0 & v_{r} & & & & \\
& & & & v_{l} & 0 & 0 & 0 \\
& \multicolumn{2}{c}{\mathbf{O}} & & 0 & v_{l} & -2 c_{l}^{2} & 2 c_{l}^{2} \\
& & & & 0 & -\frac{1}{4} & v_{l} & 0 \\
& & & & 0 & \frac{1}{4} & 0 & v_{l}
\end{array}\right), \\
& \overline{\mathcal{A}}_{2}:=\left(\begin{array}{cccccccc}
0 & 0 & 0 & 0 & & & & \\
0 & 0 & 0 & 0 & & & & \\
0 & 0 & -c_{r} & 0 & & & & \\
0 & 0 & 0 & c_{r} & & & & \\
& & & & 0 & 0 & 0 & 0 \\
& & & & 0 & 0 & 0 & 0 \\
& & & & 0 & 0 & c_{l} & 0 \\
& & & & 0 & 0 & 0 & -c_{l}
\end{array}\right)
\end{aligned}
$$

as well as

$$
\begin{aligned}
& \underline{b}=\left(\begin{array}{rr}
0 & v_{r}-v_{l} \\
1 & v_{r} \\
0 & 0
\end{array}\right)=\left(\begin{array}{rrr}
0 & 2 v_{r} \\
1 & v_{r} \\
0 & 0
\end{array}\right), \\
& \underline{M}=\left(\begin{array}{lrrr}
-2 c_{r} & -2 c_{r} & 2 c_{l} & 2 c_{l} \\
-2 c_{r} & -2 c_{r} & 0 & 0 \\
-2\left(m_{r}+n_{r}\right) & 2\left(m_{r}+n_{r}\right) & 2\left(m_{l}+n_{l}\right) & -2\left(m_{l}+n_{l}\right)
\end{array}\right) .
\end{aligned}
$$

Hereafter $\mathbf{O}$ stands for the $4 \times 4$ zero matrix. 
Let us further define the following $8 \times 8$ symmetric matrices:

$$
\begin{aligned}
& \mathcal{A}_{0}:=\left(\begin{array}{rrrrrrrr}
1 & 0 & 0 & 0 & & & & \\
0 & \frac{1}{4} & 0 & 0 & & & & \\
0 & 0 & 2 c_{r}^{2} & 0 & & & & \\
0 & 0 & 0 & 2 c_{r}^{2} & & & & \\
& & & & 1 & 0 & 0 & 0 \\
& & & & 0 & \frac{1}{4} & 0 & 0 \\
& & & & 0 & 0 & 2 c_{l}^{2} & 0 \\
& & & & 0 & 0 & 0 & 2 c_{l}^{2}
\end{array}\right) \\
& \mathcal{A}_{1}:=\left(\begin{array}{cccrccrr}
v_{r} & 0 & 0 & 0 & & & \\
0 & \frac{1}{4} v_{r} & -\frac{1}{2} c_{r}^{2} & \frac{1}{2} c_{r}^{2} & & & \\
0 & -\frac{1}{2} c_{r}^{2} & 2 c_{r}^{2} v_{r} & 0 & & & \\
0 & \frac{1}{2} c_{r}^{2} & 0 & 2 c_{r}^{2} v_{r} & & & & \\
& & & & v_{l} & 0 & 0 & 0 \\
& & & & 0 & \frac{1}{4} v_{l} & -\frac{1}{2} c_{l}^{2} & \frac{1}{2} c_{l}^{2} \\
& & & & 0 & -\frac{1}{2} c_{l}^{2} & 2 c_{l}^{2} v_{l} & 0 \\
& & & & 0 & \frac{1}{2} c_{l}^{2} & 0 & 2 c_{l}^{2} v_{l}
\end{array}\right), \\
& \mathcal{A}_{2}:=\left(\begin{array}{rccrrrrr}
0 & 0 & 0 & 0 & & & & \\
0 & 0 & 0 & 0 & & & & \\
0 & 0 & -2 c_{r}^{3} & 0 & & & & \\
0 & 0 & 0 & 2 c_{r}^{3} & & & & \\
& & & 0 & 0 & 0 & 0 \\
& & & 0 & 0 & 0 & 0 \\
& & & 0 & 0 & 2 c_{l}^{3} & 0 \\
& & & & 0 & 0 & 0 & -2 c_{l}^{3}
\end{array}\right) .
\end{aligned}
$$

Then, the linear problem (3.8) becomes equivalently the following symmetric system with $\mathcal{A}_{0}$ definite positive:

$$
\left\{\begin{array}{l}
\mathcal{L} W:=\mathcal{A}_{0} \partial_{t} W+\mathcal{A}_{1} \partial_{1} W+\mathcal{A}_{2} \partial_{2} W=f, \quad \text { if } x_{2}>0 \\
\mathcal{B}\left(W^{n c}, \psi\right) \equiv \overline{\mathcal{B}}\left(W^{n c}, \psi\right)=g, \quad \text { if } x_{2}=0
\end{array}\right.
$$

with new $f$ and $g$.

We remark that the kernel of $\mathcal{A}_{2}$ consists exactly of those $W$ satisfying $W^{n c}=0$ (and $W^{c}$ is arbitrary). Thus, the boundary $\left\{x_{2}=0\right\}$ is characteristic with multiplicity 2. As noted in earlier works (see e.g. $[11,34,38]$ ), we expect to lose control of the trace of $W^{c}$. However, we expect to have control of the trace of $W^{n c}$ on $\left\{x_{2}=0\right\}$, that is, $\left\|\left.W^{n c}\right|_{x_{2}=0}\right\|_{0}^{2}$ in (4.4) later.

3.2. Main result. Before stating our energy estimate for system (3.15), we need to introduce some weighted Sobolev norms since (3.15) is a hyperbolic problem with characteristic boundary and there is a loss of control on derivatives in the normal direction $\left(\frac{\partial}{\partial x_{2}}\right)$. 
First, define the half-space

$$
\Omega:=\left\{\left(t, x_{1}, x_{2}\right) \in \mathbb{R}^{3}: x_{2}>0\right\}=\mathbb{R}^{2} \times \mathbb{R}^{+} .
$$

For all real number $s$ and all $\lambda \geq 1$, define the weighted Sobolev space

$$
H_{\lambda}^{s}\left(\mathbb{R}^{2}\right):=\left\{u \in \mathcal{D}^{\prime}\left(\mathbb{R}^{2}\right): \exp (-\lambda t) u \in H^{s}\left(\mathbb{R}^{2}\right)\right\},
$$

which is equipped with the norm

$$
\|u\|_{H_{\lambda}^{s}\left(\mathbb{R}^{2}\right)}:=\|\exp (-\lambda t) u\|_{H^{s}\left(\mathbb{R}^{2}\right)} .
$$

Letting $\tilde{u}:=\exp (-\lambda t) u$, one has

$$
\|u\|_{H_{\lambda}^{s}\left(\mathbb{R}^{2}\right)} \simeq\|\tilde{u}\|_{s, \lambda}
$$

where

$$
\|v\|_{s, \lambda}^{2}:=\frac{1}{(2 \pi)^{2}} \int_{\mathbb{R}^{2}}\left(\lambda^{2}+|\xi|^{2}\right)^{s}|\hat{v}(\xi)|^{2} d \xi
$$

and $\hat{v}$ is the Fourier transform of a function $v$ defined on $\mathbb{R}^{2}$. For all integers $k$ and real $\lambda \geq 1$, one can define the space $H_{\lambda}^{k}(\Omega)$ as follows:

$$
H_{\lambda}^{k}(\Omega):=\left\{u \in \mathcal{D}^{\prime}(\Omega): \exp (-\lambda t) u \in H^{k}(\Omega)\right\} .
$$

For all $s>r$, it holds that

$$
H_{\lambda}^{s}\left(\mathbb{R}^{2}\right) \subset H_{\lambda}^{r}\left(\mathbb{R}^{2}\right), \quad\|v\|_{r, \lambda} \leq \frac{1}{\lambda^{s-r}}\|v\|_{s, \lambda} .
$$

The space $L^{2}\left(\mathbb{R}^{+} ; H_{\lambda}^{s}\left(\mathbb{R}^{2}\right)\right)$ is equipped with the norm

$$
\left|\|v \mid\|_{L^{2}\left(H_{\lambda}^{s}\right)}^{2}:=\int_{0}^{\infty}\left\|v\left(\cdot, x_{2}\right)\right\|_{H_{\lambda}^{s}\left(\mathbb{R}^{2}\right)}^{2} d x_{2} .\right.
$$

Our first main result is stated as follows.

Theorem 3.1. Let $\left(U_{r, l}, \Phi_{r, l}\right)$ be a solution to (2.21), (2.17), and (2.18) defined by (2.22) and (2.23).

(i) If

$$
v_{r}-v_{l}>\left(c_{r}^{\frac{2}{3}}+c_{l}^{\frac{2}{3}}\right)^{\frac{3}{2}} \quad \text { and } \quad v_{r}-v_{l} \neq \sqrt{2}\left(c_{r}+c_{l}\right)
$$

Then, there exists a positive constant $C$ such that, for all $\lambda \geq 1$ and for all solutions $(W, \psi) \in H_{\lambda}^{2}(\Omega) \times H_{\lambda}^{2}\left(\mathbb{R}^{2}\right)$ to $(3.15)$, the following estimate holds:

$$
\begin{aligned}
& \lambda\left\|\left|W\left\|\left.\right|_{L_{\lambda}^{2}(\Omega)} ^{2}+\right\| W^{n c}\right|_{x_{2}=0}\right\|_{L_{\lambda}^{2}\left(\mathbb{R}^{2}\right)}^{2}+\|\psi\|_{H_{\lambda}^{1}\left(\mathbb{R}^{2}\right)}^{2} \\
\leq & C\left(\frac { 1 } { \lambda ^ { 3 } } \left\|\left|\mathcal{L} W\left\|\left.\right|_{L^{2}\left(H_{\lambda}^{1}\right)} ^{2}+\frac{1}{\lambda^{2}}\right\| \mathcal{B}(W, \psi) \|_{H_{\lambda}^{1}\left(\mathbb{R}^{2}\right)}^{2}\right) .\right.\right.
\end{aligned}
$$

(ii) If

$$
v_{r}-v_{l}=\sqrt{2}\left(c_{r}+c_{l}\right)
$$


then there exists a positive constant $C$ such that, for all $\lambda \geq 1$ and for all solutions $(W, \psi) \in H_{\lambda}^{3}(\Omega) \times H_{\lambda}^{3}\left(\mathbb{R}^{2}\right)$ to (3.15), the following estimate holds:

$$
\begin{aligned}
& \lambda\left\|\left|W\left\|\left.\right|_{L_{\lambda}^{2}(\Omega)} ^{2}+\right\| W^{n c}\right|_{x_{2}=0}\right\|_{L_{\lambda}^{2}\left(\mathbb{R}^{2}\right)}^{2}+\|\psi\|_{H_{\lambda}^{1}\left(\mathbb{R}^{2}\right)}^{2} \\
\leq & C\left(\frac { 1 } { \lambda ^ { 5 } } \left\|\left|\mathcal{L} W\left\|\left.\right|_{L^{2}\left(H_{\lambda}^{2}\right)} ^{2}+\frac{1}{\lambda^{4}}\right\| \mathcal{B}(W, \psi) \|_{H_{\lambda}^{2}\left(\mathbb{R}^{2}\right)}^{2}\right) .\right.\right.
\end{aligned}
$$

Here, $c_{r, l}$ are defined by (3.7).

We shall prove Theorem 3.1 by a normal mode analysis of (3.15). Introduce the new unknown functions:

$$
\tilde{W}:=\exp (-\lambda t) W, \quad \tilde{\psi}:=\exp (-\lambda t) \psi
$$

Then, (3.15) is equivalent to

$$
\left\{\begin{array}{l}
\mathcal{L}^{\lambda} \tilde{W}:=\lambda \mathcal{A}_{0} \tilde{W}+\mathcal{L} \tilde{W}=\exp (-\lambda t) f, \quad \text { if } x_{2}>0 \\
\mathcal{B}^{\lambda}\left(\tilde{W}^{n c}, \tilde{\psi}\right)=\left.\underline{M} \tilde{W}^{n c}\right|_{x_{2}=0}+\underline{b}\left(\begin{array}{c}
\lambda \tilde{\psi}+\partial_{t} \tilde{\psi} \\
\partial_{1} \tilde{\psi}
\end{array}\right)=\exp (-\lambda t) g, \quad \text { if } x_{2}=0
\end{array}\right.
$$

We can rewrite Theorem 3.1 equivalently as the following.

THEOREM 3.2. (i) Assume that (3.16) holds. Then, there exists a positive constant $C$ such that, for all $\lambda \geq 1$ and for all $(\tilde{W}, \tilde{\psi}) \in H^{2}(\Omega) \times H^{2}\left(\mathbb{R}^{2}\right)$, the following estimate holds:

$$
\begin{gathered}
\lambda\left\|\left|\tilde{W}\left\|\left.\right|_{0} ^{2}+\right\| \tilde{W}^{n c}\right|_{x_{2}=0}\right\|_{0}^{2}+\|\tilde{\psi}\|_{1, \lambda}^{2} \\
\leq C\left(\frac { 1 } { \lambda ^ { 3 } } \left\|\left|\mathcal{L}^{\lambda} \tilde{W}\left\|\left.\right|_{1, \lambda} ^{2}+\frac{1}{\lambda^{2}}\right\| \mathcal{B}^{\lambda}(\tilde{W}, \tilde{\psi}) \|_{1, \lambda}^{2}\right) .\right.\right.
\end{gathered}
$$

(ii) Assume that (3.18) holds. Then, there exists a positive constant $C$ such that for all $\lambda \geq 1$ and for all $(\tilde{W}, \tilde{\psi}) \in H^{3}(\Omega) \times H^{3}\left(\mathbb{R}^{2}\right)$, the following estimate holds:

$$
\begin{aligned}
& \lambda\left\|\left|\tilde{W}\left\|\left.\right|_{0} ^{2}+\right\| \tilde{W}^{n c}\right|_{x_{2}=0}\right\|_{0}^{2}+\|\tilde{\psi}\|_{1, \lambda}^{2} \\
\leq & C\left(\frac { 1 } { \lambda ^ { 5 } } \left\|\left|\mathcal{L}^{\lambda} \tilde{W}\left\|\left.\right|_{2, \lambda} ^{2}+\frac{1}{\lambda^{4}}\right\| \mathcal{B}^{\lambda}(\tilde{W}, \tilde{\psi}) \|_{2, \lambda}^{2}\right) .\right.\right.
\end{aligned}
$$

In (3.21) and (3.22), we have used the following notation for $v \in L^{2}\left(\mathbb{R}^{+} ; H^{s}\left(\mathbb{R}^{2}\right)\right)$ :

$$
\|\mid v\|_{s, \lambda}^{2}:=\int_{0}^{+\infty}\left\|v\left(\cdot, x_{2}\right)\right\|_{s, \lambda}^{2} d x_{2} .
$$

For instance, $\|\left.|\cdot|\right|_{0, \lambda} ^{2}$ is the usual norm on $L^{2}(\Omega)$ and does not involve $\lambda$, so we shall denote it by $\left\||\cdot \||_{0}^{2}\right.$. The norm $\||\cdot \||_{1, \lambda}^{2}$ is the weighted norm on $L^{2}\left(\mathbb{R}^{+} ; H^{1}\left(\mathbb{R}^{2}\right)\right)$.

\section{Proof of Theorem 3.2}

This section is devoted to the proof of Theorem 3.2. To simplify the notation, we shall drop the tildes from the unknowns $\tilde{W}, \tilde{\psi}$. As in [11], by introducing an auxiliary problem with maximally dissipative boundary conditions, one can show that it is sufficient to prove estimates (3.21) and (3.22) for the system with zero interior source term

$$
\lambda \mathcal{A}_{0} W+\mathcal{A}_{0} \partial_{t} W+\mathcal{A}_{1} \partial_{1} W+\mathcal{A}_{2} \partial_{2} W=0
$$


in the interior domain $\Omega$, as well as the following boundary conditions:

$$
\left.\underline{M} W^{n c}\right|_{x_{2}=0}+\underline{b}\left(\begin{array}{c}
\lambda \psi+\partial_{t} \psi \\
\partial_{1} \psi
\end{array}\right)=g \text {, on } x_{2}=0 .
$$

Recall that all matrices $\mathcal{A}_{j}(j=0,1,2)$ are symmetric and that $\mathcal{A}_{0}$ is positive definite (see (3.12)). We take the scalar product of (4.1) with $W$ and integrating over $\Omega$ yield the following inequality:

$$
\lambda\left\|\left|W\left\|\left.\right|_{0} ^{2} \leq C\right\| W^{n c}\right|_{x_{2}=0}\right\|_{0}^{2} .
$$

Consequently, in order to obtain (3.21) and (3.22), it is sufficient to derive the following estimates:

$$
\begin{gathered}
\left\|\left.W^{n c}\right|_{x_{2}=0}\right\|_{0}^{2}+\|\psi\|_{1, \lambda}^{2} \leq \frac{C}{\lambda^{2}}\|g\|_{1, \lambda}^{2}, \\
\left\|\left.W^{n c}\right|_{x_{2}=0}\right\|_{0}^{2}+\|\psi\|_{1, \lambda}^{2} \leq \frac{C}{\lambda^{4}}\|g\|_{2, \lambda}^{2},
\end{gathered}
$$

which can be further reduced to estimate the $L^{2}$-norm of the trace of $W^{n c}$ in the Sobolev norm of $g$ by "eliminating" the front $\psi$ in the boundary conditions (4.2) as in [11]. In the next subsections, we shall perform the normal mode analysis in detail and construct a symbolic symmetrizer.

4.1. Elimination of the front. First, we apply the Fourier transform in $\left(t, x_{1}\right)$ on (4.1) and (4.2). Denote the dual variables by $(\delta, \eta)$ and define $\tau:=\lambda+i \delta$. Then, we obtain the following system of ordinary differential equations (ODEs):

$$
\begin{aligned}
& \left(\tau \mathcal{A}_{0}+i \eta \mathcal{A}_{1}\right) \widehat{W}+\mathcal{A}_{2} \frac{d \widehat{W}}{d x_{2}}=0, \quad x_{2}>0, \\
& b(\tau, \eta) \widehat{\psi}+\underline{M} \widehat{W}^{n c}(0)=\widehat{g},
\end{aligned}
$$

where $b(\tau, \eta)$ is simply defined by

$$
b(\tau, \eta):=\underline{b}\left(\begin{array}{c}
\tau \\
i \eta
\end{array}\right)=\left(\begin{array}{c}
2 i v_{r} \eta \\
\tau+i v_{r} \eta \\
0
\end{array}\right) .
$$

Recall that $\underline{b}$ and $\underline{M}$ are defined by (3.11). Observe that $b(\tau, \eta)$ is homogeneous of degree 1 with respect to $(\tau, \eta)$. Define the hemisphere

$$
\Sigma:=\left\{(\tau, \eta) \in \mathbb{C} \times \mathbb{R}:|\tau|^{2}+v_{r}^{2} \eta^{2}=1 \text { and } \mathfrak{R} \tau \geq 0\right\},
$$

where $\mathfrak{R} \tau$ is the real part of $\tau$, and denote by $\Xi$ the set

$$
\Xi:=\left\{(\lambda, \delta, \eta) \in[0,+\infty) \times \mathbb{R}^{2}:(\lambda, \delta, \eta) \neq(0,0,0)\right\}=(0,+\infty) \cdot \Sigma .
$$

We always identify $(\lambda, \delta) \in \mathbb{R}^{2}$ with $\tau=\lambda+i \delta \in \mathbb{C}$. We remark that a symbolic symmetrizer $r(\tau, \eta)$ of (4.15) as a homogeneous function of degree zero with respect to $(\tau, \eta) \in \Xi$ will be constructed. In fact, it is enough to construct $r(\tau, \eta)$ in the unit hemisphere $\Sigma$. Since $\Sigma$ is a compact set, by a smooth partition of unity, it can be reduced to construct $r(\tau, \eta)$ in a neighborhood of each point of $\Sigma$. 
One crucial note is that the symbol $b(\tau, \eta)$ is elliptic, that is, it does not vanish on the closed hemisphere $\Sigma$. Similar to [11], we can choose the $c^{\infty}$ mapping $Q$ on $\Sigma$ as follows:

$$
\forall(\tau, \eta) \in \Sigma, \quad Q(\tau, \eta):=\left(\begin{array}{lcr}
0 & 0 & \frac{1}{2(\bar{m}+\bar{n})} \\
\frac{1}{2}\left(\tau+i v_{r} \eta\right) & -i v_{r} \eta & 0 \\
-2 i v_{r} \eta & \bar{\tau}-i v_{r} \eta & 0
\end{array}\right),
$$

which is homogeneous of degree zero. For reducing the boundary matrix $\beta(\tau, \eta)$ in $(4.13)$ later to the same form as that of [44] and directly employing their parts of construction on symmetrizer, we may choose

$$
\frac{1}{2(\bar{m}+\bar{n})}=\frac{1}{2\left(m_{r}+n_{r}\right)}=\frac{1}{2\left(m_{l}+n_{l}\right)}
$$

in the first row of $Q(\tau, \eta)$ and (4.9) can truely be satisfied due to (2.23). Then one can easily check

$$
Q(\tau, \eta) b(\tau, \eta)=\left(\begin{array}{c}
0 \\
0 \\
\theta(\tau, \eta)
\end{array}\right)
$$

Here $\theta$ is $C^{\infty}$, homogeneous of degree 1 and given by

$$
\theta(\tau, \eta):=\left|\tau+i v_{r} \eta\right|^{2}+4 v_{r}^{2} \eta^{2}, \quad(\tau, \eta) \in \Sigma,
$$

satisfying the lower bound

$$
\min _{(\tau, \eta) \in \Sigma}|\theta(\tau, \eta)|>0
$$

Note that the last row of $Q(\tau, \eta)$ is nothing but $b(\tau, \eta)^{*}$ when $(\tau, \eta) \in \Sigma$ and $Q$ can be extended to $\Xi$ by homogeneity.

Let us multiply the boundary conditions in (4.6) by the matrix $Q(\tau, \eta)$. By repeating the arguments of [11], the elliptic estimate $(4.10)$ for $\theta(\tau, \eta)$ yields the control of the front:

$$
\begin{aligned}
\|\psi\|_{1, \lambda}^{2} & \leq C\left(\left\|\left.W^{n c}\right|_{x_{2}=0}\right\|_{0}^{2}+\|g\|_{0}^{2}\right) \leq C\left(\left\|\left.W^{n c}\right|_{x_{2}=0}\right\|_{0}^{2}+\frac{1}{\lambda^{2}}\|g\|_{1, \lambda}^{2}\right) \\
& \leq C\left(\left\|\left.W^{n c}\right|_{x_{2}=0}\right\|_{0}^{2}+\frac{1}{\lambda^{4}}\|g\|_{2, \lambda}^{2}\right) .
\end{aligned}
$$

Therefore, in order to obtain (4.4) and (4.5), it is sufficient to derive an estimate on the trace of $W^{n c}$. Consequently, we focus on the reduced algebraic-differential equation problem

$$
\begin{aligned}
& \left(\tau \mathcal{A}_{0}+i \eta \mathcal{A}_{1}\right) \widehat{W}+\mathcal{A}_{2} \frac{d \widehat{W}}{d x_{2}}=0, \quad x_{2}>0, \\
& \beta(\tau, \eta) \widehat{W^{n c}}(0)=\hat{h},
\end{aligned}
$$

and we shall derive an estimate for $\widehat{W^{n}}(0)$. Here the source term $\widehat{h} \in \mathbb{C}^{2}$ can be estimated by $\widehat{g}$ and

$$
\beta(\tau, \eta)=\left(\begin{array}{lccr}
-1 & 1 & 1 & -1 \\
-c_{r}\left(\tau-i v_{r} \eta\right) & -c_{r}\left(\tau-i v_{r} \eta\right) & c_{l}\left(\tau+i v_{r} \eta\right) & c_{l}\left(\tau+i v_{r} \eta\right)
\end{array}\right)
$$


as in [44] because of our choice of $Q$ in (4.8), but the precise expressions of $c_{r, l}$ defined by (3.7) in this paper are different from those in [44].

Next, we shall recall that, under the assumption made in theorems 3.1 and 3.2 , the above problem satisfies the Kreiss-Lopatinskii condition but violates the uniform Kreiss-Lopatinskii condition.

4.2. The normal mode analysis. Due to the singularity of the matrix $\mathcal{A}_{2}$, some equations in (4.12) do not involve derivation with respect to the normal variable $x_{2}$. The second and sixth equations in (4.12) read

$$
\begin{aligned}
& \frac{1}{4}\left(\tau+i v_{r} \eta\right) \hat{W}_{2}-\frac{1}{2} i c_{r}^{2} \eta \hat{W}_{3}+\frac{1}{2} i c_{r}^{2} \eta \hat{W}_{4}=0, \\
& \frac{1}{4}\left(\tau+i v_{l} \eta\right) \hat{W}_{6}-\frac{1}{2} i c_{l}^{2} \eta \hat{W}_{7}+\frac{1}{2} i c_{l}^{2} \eta \hat{W}_{8}=0
\end{aligned}
$$

based on the expression

$$
\tau \mathcal{A}_{0}+i \eta \mathcal{A}_{1}=\left(\begin{array}{lr}
\left(\tau \mathcal{A}_{0}+i \eta \mathcal{A}_{1}\right)_{r} & \mathbf{0} \\
\mathbf{0} & \left(\tau \mathcal{A}_{0}+i \eta \mathcal{A}_{1}\right)_{l}
\end{array}\right)
$$

and

$$
\left(\tau \mathcal{A}_{0}+i \eta \mathcal{A}_{1}\right)_{r, l}=\left(\begin{array}{cccr}
\tau+i v_{r, l} \eta & 0 & 0 & 0 \\
0 & \frac{1}{4}\left(\tau+i v_{r, l} \eta\right) & -\frac{1}{2} i c_{r, l}^{2} \eta & \frac{1}{2} i c_{r, l}^{2} \eta \\
0 & -\frac{1}{2} i c_{r, l}^{2} \eta & 2 c_{r, l}^{2}\left(\tau+i v_{r, l} \eta\right) & 0 \\
0 & \frac{1}{2} i c_{r, l}^{2} \eta & 0 & 2 c_{r, l}^{2}\left(\tau+i v_{r, l} \eta\right)
\end{array}\right)
$$

Thus we obtain an expression for $\widehat{W}_{2}$ and $\widehat{W}_{6}$ that we can substitute in the third, fourth, seventh, and eighth equations in (4.12). This operation yields a system of ordinary differential equations of the following form:

$$
\left\{\begin{array}{l}
\frac{d \widehat{W^{n} c}}{d x_{2}}=\mathcal{A}(\tau, \eta) \widehat{W^{n c}}, \quad x_{2}>0, \\
\beta(\tau, \eta) \widehat{W^{n} c}(0)=\hat{h}, \quad x_{2}=0 .
\end{array}\right.
$$

The matrix $\mathcal{A}(\tau, \eta)$ in (4.15) is given by the same form as in $[11,44]$ :

$$
\begin{aligned}
& \mathcal{A}(\tau, \eta):=\left(\begin{array}{cccc}
\mu_{r} & -m_{r} & 0 & 0 \\
m_{r} & -\mu_{r} & 0 & 0 \\
0 & 0 & -\mu_{l} & m_{l} \\
0 & 0 & -m_{l} & \mu_{l}
\end{array}\right), \quad \text { with } \\
& \mu_{r, l}:=\frac{\left(1 / c_{r, l}\right)\left(\tau+i v_{r, l} \eta\right)^{2}+\left(c_{r, l} / 2\right) \eta^{2}}{\tau+i v_{r, l} \eta}, \\
& m_{r, l}:=\frac{\left(c_{r, l} / 2\right) \eta^{2}}{\tau+i v_{r, l} \eta}
\end{aligned}
$$

where $c_{r, l}$ are defined by (3.7). By computing the eigenvalues and the stable subspace of $\mathcal{A}(\tau, \eta)$, the theoretical results in [25,27,39] apply. The following lemma of [11] gives an expression of the stable subspace.

Lemma 4.1 ( $[11]$, Lemma 4.2). Let $\tau \in \mathbb{C}$ and $\eta \in \mathbb{R}$, with $\mathfrak{R} \tau>0$ and $(\tau, \eta) \in \Sigma$. The eigenvalues of $\mathcal{A}(\tau, \eta)$ are the roots $\omega$ of the dispersion relations

$$
\begin{aligned}
& \omega_{r}^{2}=\mu_{r}^{2}-m_{r}^{2}=\frac{1}{c_{r}^{2}}\left(\tau+i v_{r} \eta\right)^{2}+\eta^{2}, \\
& \omega_{l}^{2}=\mu_{l}^{2}-m_{l}^{2}=\frac{1}{c_{l}^{2}}\left(\tau+i v_{l} \eta\right)^{2}+\eta^{2} .
\end{aligned}
$$


In particular, (4.17) $)_{1}$ (resp. (4.17) 2 ) admits a unique root $\omega_{r}$ (resp. $\omega_{l}$ ) of negative real part. The other root of $(4.17)_{1}$ (resp. $\left.(4.17)_{2}\right)$ is $-\omega_{r}$ (resp. $\left.-\omega_{l}\right)$ and has positive real part. The stable subspace $E^{-}(\tau, \eta)$ of $\mathcal{A}(\tau, \eta)$ has dimension 2 and is spanned by the following two vectors:

$$
\begin{aligned}
& E_{r}(\tau, \eta):=\left(\frac{c_{r}}{2} \eta^{2}, \frac{1}{c_{r}}\left(\tau+i v_{r} \eta\right)^{2}+\frac{c_{r}}{2} \eta^{2}-\left(\tau+i v_{r} \eta\right) \omega_{r}, 0,0\right)^{\top} \\
& E_{l}(\tau, \eta):=\left(0,0, \frac{1}{c_{l}}\left(\tau+i v_{r} \eta\right)^{2}+\frac{c_{l}}{2} \eta^{2}-\left(\tau+i v_{r} \eta\right) \omega_{r}, \frac{c_{l}}{2} \eta^{2}\right)^{\top} .
\end{aligned}
$$

Both $\omega_{r}$ and $\omega_{l}$ admit a continuous extension to any point $(\tau, \eta)$ such that $\mathfrak{R} \tau=0$ and $(\tau, \eta) \in \Sigma$. This allows us to extend both vectors $E_{r}$ and $E_{l}$ in (4.18) to the whole hemisphere $\Sigma$. Those two vectors are linearly independent for any value of $(\tau, \eta) \in \Sigma$.

The symbol $\mathcal{A}(\tau, \eta)$ is diagonalizable as long as both $\omega_{r}$ and $\omega_{l}$ do not vanish, that is, when $\tau \neq\left( \pm v_{r} \pm c_{r, l}\right)$ i $\eta$. Away from such points, $\mathcal{A}$ admits a $C^{\infty}$ basis of eigenvectors.

Following Majda and Osher $[11,38]$, we define the Lopatinskii determinant associated with the boundary conditions $\beta$ in the following way:

$$
\Delta(\tau, \eta):=\operatorname{det}\left[\beta(\tau, \eta)\left(E_{r}(\tau, \eta) E_{l}(\tau, \eta)\right)\right],
$$

with $\beta$ defined by (4.13) and $\left(E_{r}, E_{l}\right)$ defined by (4.18). We emphasize that the Lopatinskii determinant $\Delta$ is defined on the whole hemisphere $\Sigma$ and is continuous with respect to $(\tau, \eta)$. The first step in proving an energy estimate for (4.15) is to determine whether $\Delta$ vanishes on $\Sigma$. The answer is given in the following result.

Proposition 4.2 (see [44], Proposition 3.4). Assume that the condition

$$
v_{r}-v_{l}=2 v_{r}>\left(c_{r}^{\frac{2}{3}}+c_{l}^{\frac{2}{3}}\right)^{\frac{3}{2}}
$$

holds.

(a) If $c_{r}=c_{l}:=c$, then there exists a positive number $V_{1}$ such that for any $(\tau, \eta) \in \Sigma$, one has $\Delta(\tau, \eta)=0$ if and only if

$$
\tau=0 \quad \text { or } \quad \tau= \pm i V_{1} \eta .
$$

Each of the preceding roots of $\Delta(\tau, \eta)=0$ is simple, namely, if $\left(\tau_{0}, \eta_{0}\right)$ is any of the points above, there exists an open neighborhood $\mathcal{V}$ of $\left(\tau_{0}, \eta_{0}\right)$ in $\Sigma$ and a $C^{\infty}$ function $h$ defined on $\mathcal{V}$ such that, for all $(\tau, \eta) \in \mathcal{V}$, one has $\Delta(\tau, \eta)=\left(\tau-\tau_{0}\right) h(\tau, \eta)$ and $h\left(\tau_{0}, \eta_{0}\right) \neq 0$.

(b) If $c_{r} \neq c_{l}$, then there exist two positive numbers $X_{2}, X_{3}$ satisfying

$$
c_{r}-v_{r}<c_{r} X_{2}<c_{r} X_{3}<-c_{l}+v_{r},
$$

such that $\Delta(\tau, \eta)=0$ for $(\tau, \eta) \in \Sigma$ if and only if

$$
\tau=i q v_{r} \eta \quad \text { or } \tau=i c_{r} X_{2} q \text { or } \tau=i c_{r} X_{3} q
$$

where $q:=\frac{c_{r}-c_{l}}{c_{r}+c_{l}}$.

For $v_{r} \neq \frac{c_{r}+c_{l}}{\sqrt{2}}$, each of the preceding roots of $\Delta(\tau, \eta)=0$ is simple. When $v_{r}=\frac{c_{r}+c_{l}}{\sqrt{2}}$, one (and only one) of the two identities below holds:

$$
q v_{r}=c_{r} X_{2} \text { or } q v_{r}=c_{r} X_{3} .
$$


Hence, each of the roots $\left(i q v_{r} \eta, \eta\right) \in \Sigma$ of $\Delta(\tau, \eta)=0$ is quadratic. This means that to every point $\left(i q v_{r} \eta_{0}, \eta_{0}\right) \in \Sigma$, there corresponds an open neighborhood $\mathcal{V}$ in $\Sigma$ and a $C^{\infty}$ function $h$ on $\mathcal{V}$ such that

$$
\Delta(\tau, \eta)=\left(\tau-i q v_{r} \eta_{0}\right)^{2} h(\tau, \eta), \quad \forall(\tau, \eta) \in \mathcal{V},
$$

and $h\left(i q v_{r} \eta_{0}, \eta_{0}\right) \neq 0$. The other root of $\Delta(\tau, \eta)=0$ is simple.

4.3. Construction of the symmetrizer. This subsection will be entirely devoted to constructing a symbolic symmetrizer $r(\tau, \eta)$, which is a homogeneous function of degree zero with respect to $(\tau, \eta) \in \Xi$. As remarked earlier, it is sufficient to construct $r(\tau, \eta)$ in a neighborhood of each point of $\Sigma$. The analysis performed in Section 4.2 shows that we have to consider five different classes of frequencies $(\tau, \eta) \in \Sigma$ in the construction of $r(\tau, \eta)$ :

(C1) the interior points $\left(\tau_{0}, \eta_{0}\right)$ of $\Sigma$ such that $\mathfrak{R} \tau_{0}>0$;

(C2) the boundary points $\left(\tau_{0}, \eta_{0}\right)$ of $\Sigma$ where $\mathcal{A}\left(\tau_{0}, \eta_{0}\right)$ is diagonalizable and the Lopatinskii condition is satisfied at $\left(\tau_{0}, \eta_{0}\right)$ (namely, $\Delta\left(\tau_{0}, \eta_{0}\right) \neq 0$ );

(C3) the boundary points $\left(\tau_{0}, \eta_{0}\right)$ where $\mathcal{A}\left(\tau_{0}, \eta_{0}\right)$ is diagonalizable but the Lopatinskii condition breaks down at $\left(\tau_{0}, \eta_{0}\right)$ (i.e. $\left.\Delta\left(\tau_{0}, \eta_{0}\right)=0\right)$;

(C4) Those points $\left(\tau_{0}, \eta_{0}\right)$ where $\mathcal{A}\left(\tau_{0}, \eta_{0}\right)$ is not diagonalizable, that is, $\tau_{0}=\left( \pm v_{r} \pm\right.$ $\left.c_{r, l}\right) i \eta_{0}\left(\eta_{0} \neq 0\right)$, and in this case Proposition 4.2 asserts that the Lopatinskii condition is satisfied at $\left(\tau_{0}, \eta_{0}\right)$; and

(C5) Those points $\left(\tau_{0}, \eta_{0}\right)$ that are the poles of $\mathcal{A}$, that is, $\tau_{0}= \pm i v_{r} \eta_{0}$, and at those points, the Lopatinskii condition is satisfied.

The construction of the symmetrizer is microlocal and is performed near each point $\left(\tau_{0}, \eta_{0}\right) \in \Sigma$. For a point belonging to the above classes $(\mathrm{C} 1),(\mathrm{C} 2)$, and $(\mathrm{C} 4)$, the construction is very similar to the corresponding one of sections $4.4,4.5$, and 4.7 in [11]. It can be proved that, if $\left(\tau_{0}, \eta_{0}\right)$ is a point in one of the above classes, there exist a neighborhood $\mathcal{V}$ of $\left(\tau_{0}, \eta_{0}\right)$ and two $C^{\infty}$ mappings $T: \mathcal{V} \rightarrow G L_{4}(\mathbb{C})$ and $r: \mathcal{V} \rightarrow M_{4 \times 4(\mathbb{C})}$ such that, for all $(\tau, \eta) \in \mathcal{V}, r(\tau, \eta)$ is Hermitian and homogeneous of degree zero with respect to $(\tau, \eta)$, and such that the estimates

$$
\begin{aligned}
& \Re\left(r(\tau, \eta) T(\tau, \eta) \mathcal{A}(\tau, \eta) T^{-1}(\tau, \eta)\right) \geq \kappa I_{4 \times 4} \geq \kappa \lambda I_{4 \times 4}, \quad \forall(\tau, \eta) \in \mathcal{V}, \\
& r(\tau, \eta)+C\left(\beta(\tau, \eta) T(\tau, \eta)^{-1}\right)^{*} \beta(\tau, \eta) T(\tau, \eta)^{-1} \geq I_{4 \times 4}, \quad \forall(\tau, \eta) \in \mathcal{V},
\end{aligned}
$$

hold, where $I_{4 \times 4}$ denotes the identity matrix of order 4 , and $C, \kappa$ are suitable positive constants. On the left-hand side of $(4.20)$, we use the notation $\mathfrak{R} M:=\frac{M+M^{*}}{2}$ for every complex square matrix $M$. Here, $M^{*}=\bar{M}^{T}$ is the conjugate transpose.

For the case of points belonging to the class (C3), as in [44] the symmetrizer is defined in a neighborhood of $\left(\tau_{0}, \eta_{0}\right)$ by

$$
r(\tau, \eta):=\operatorname{diag}\left(-\lambda^{2 \nu_{0}},-\lambda^{2 \nu_{0}}, K, K\right),
$$

where $\nu_{0}=1$ or $\nu_{0}=2$ correspond to $v_{r} \neq \frac{c_{r}+c_{l}}{2}$ or $v_{r}=\frac{c_{r}+c_{l}}{2}$, respectively, and $K$ is a constant to be chosen large enough. The matrix $r(\tau, \eta)$ above is Hermitian and satisfies

$$
\begin{aligned}
& \mathfrak{R}\left(r(\tau, \eta) T(\tau, \eta) \mathcal{A}(\tau, \eta) T^{-1}(\tau, \eta)\right) \geq \kappa \lambda \operatorname{diag}\left(-\lambda^{2 \nu_{0}},-\lambda^{2 \nu_{0}}, K, K\right), \\
& r(\tau, \eta)+C\left(\beta(\tau, \eta) T(\tau, \eta)^{-1}\right)^{*} \beta(\tau, \eta) T(\tau, \eta)^{-1} \geq \lambda^{2 \nu_{0}} I_{4 \times 4}
\end{aligned}
$$

for all $(\tau, \eta) \in \mathcal{V}$ and suitable positive constants $\kappa, C>0$. 
We now consider the last case (C5), which is $\left(\tau_{0}, \eta_{0}\right) \in \Sigma$ with $\tau_{0}=-i v_{r} \eta_{0}$. As in [11], we have to go back to the original system:

$$
\begin{aligned}
& \left(\tau \mathcal{A}_{0}+i \eta \mathcal{A}_{1}\right) \widehat{W}+\mathcal{A}_{2} \frac{d \widehat{W}}{d x_{2}}=0, \quad x_{2}>0, \\
& \beta(\tau, \eta) \widehat{W^{n c}}(0)=\hat{h}
\end{aligned}
$$

Note that the matrices $\tau \mathcal{A}_{0}+i \eta \mathcal{A}_{1}$ and $\mathcal{A}_{2}$ are different from those of [11]. The idea now is to perform some manipulations on the rows of these two matrices so that $(4.22)_{1}$ is transformed into an "almost diagonal" system of differential equations. By direct calculations similar to those in [11], we can choose both $C^{\infty}$ matrices $\mathcal{S}$ and $\mathcal{T}$ on the whole neighborhood $\mathcal{V}$ as follows:

$$
S(\tau, \eta):=\left(\begin{array}{lr}
S_{r}(\tau, \eta) & \mathbf{O} \\
\mathbf{O} & S_{l}(\tau, \eta)
\end{array}\right), \quad \mathcal{T}(\tau, \eta):=\left(\begin{array}{lr}
\mathcal{T}_{r}(\tau, \eta) & \mathbf{O} \\
\mathbf{O} & \mathcal{T}_{l}(\tau, \eta)
\end{array}\right)
$$

where

$$
\begin{aligned}
& S_{r}(\tau, \eta)=\left(\begin{array}{cccc}
1 & 0 & 0 & 0 \\
0 & 1 & 0 & 0 \\
0 & 0 & 1 & 0 \\
0 & \frac{2 i}{c_{r}^{3} \eta}\left(\tau+i v_{r} \eta-c_{r} \omega_{r}\right) & \frac{\xi_{r}}{c_{r}^{3} \eta^{2}} & \frac{1}{2 c_{r}^{3}}
\end{array}\right) \\
& S_{l}(\tau, \eta)=\left(\begin{array}{cccc}
1 & 0 & 0 & 0 \\
0 & 1 & 0 & 0 \\
0 & -\frac{i\left(\tau+i v_{l} \eta+c_{l} \omega_{l}\right)}{c_{l}^{3} \eta \omega_{l}\left(\tau+i v_{l} \eta\right)} & \frac{1}{4 c_{l}^{3} \omega_{l}\left(\tau+i v_{l} \eta\right)} & \frac{\xi_{l}^{+}}{2 c_{l}^{4} \eta^{2} \omega_{l}\left(\tau+i v_{l} \eta\right)} \\
0 & -\frac{i\left(\tau+i v_{l} \eta-c_{l} \omega_{l}\right)}{c_{l}^{3} \eta \omega_{l}\left(\tau+i v_{l} \eta\right)} & \frac{1}{4 c_{l}^{3} \omega_{l}\left(\tau+i v_{l} \eta\right)} & \frac{\xi_{l}^{-}}{2 c_{l}^{4} \eta^{2} \omega_{l}\left(\tau+i v_{l} \eta\right)}
\end{array}\right), \\
& \mathcal{T}_{r}(\tau, \eta):=\left(\begin{array}{cccc}
1 & 0 & 0 & 0 \\
0 & 1 & -i c_{r} \eta\left(\tau+i v_{r} \eta-c_{r} \omega_{r}\right) & 0 \\
0 & 0 & \frac{c_{r}}{2} \eta^{2} & 0 \\
0 & 0 & \left(\tau+i v_{r} \eta\right)\left(\mu_{r}-\omega_{r}\right) & 1
\end{array}\right) \\
& \mathcal{T}_{l}(\tau, \eta):=\left(\begin{array}{cccr}
1 & 0 & 0 & 0 \\
0 & 1 & i c_{l} \eta\left(\tau+i v_{l} \eta-c_{l} \omega_{l}\right) & i c_{l} \eta\left(\tau+i v_{l} \eta+c_{l} \omega_{l}\right) \\
0 & 0 & \left(\tau+i v_{l} \eta\right)\left(\mu_{l}-\omega_{l}\right) & \left(\tau+i v_{l} \eta\right)\left(\mu_{l}+\omega_{l}\right) \\
0 & 0 & \frac{c_{l}}{2} \eta^{2} & \frac{c_{l}}{2} \eta^{2}
\end{array}\right),
\end{aligned}
$$

and

$$
\begin{aligned}
\xi_{r}: & =\left(\tau+i v_{r} \eta\right)\left(\mu_{r}-\omega_{r}\right)=\left(\tau+i v_{r} \eta\right) \mu_{r}-\left(\tau+i v_{r} \eta\right) \omega_{r} \\
& =\frac{1}{c_{r}}\left(\tau+i v_{r} \eta\right)^{2}+\frac{c_{r}}{2} \eta^{2}-\left(\tau+i v_{r} \eta\right) \omega_{r} \\
\xi_{l}^{ \pm}: & =\left(\tau+i v_{l} \eta\right)\left(\mu_{l} \pm \omega_{l}\right)=\left(\tau+i v_{l} \eta\right) \mu_{l} \pm\left(\tau+i v_{l} \eta\right) \omega_{l} \\
& =\frac{1}{c_{l}}\left(\tau+i v_{l} \eta\right)^{2}+\frac{c_{l}}{2} \eta^{2} \pm\left(\tau+i v_{l} \eta\right) \omega_{l}
\end{aligned}
$$


It is now easy to derive the following equalities for all $(\tau, \eta)$ in $\mathcal{V}$ :

$$
\begin{aligned}
& S(\tau, \eta) \mathcal{A}_{2} \mathcal{T}(\tau, \eta)=\operatorname{diag}\left(0,0,-c_{r}^{4} \eta^{2}, 1,0,0,-1,1\right) \\
& S(\tau, \eta)\left(\tau \mathcal{A}_{0}+i \eta \mathcal{A}_{1}\right) \mathcal{T}(\tau, \eta) \\
= & \left(\begin{array}{ccc}
S_{r}(\tau, \eta)\left(\tau \mathcal{A}_{0}+i \eta \mathcal{A}_{1}\right)_{r} \mathcal{T}_{r}(\tau, \eta) & \mathbf{O} \\
\mathbf{O} & & S_{l}(\tau, \eta)\left(\tau \mathcal{A}_{0}+i \eta \mathcal{A}_{1}\right)_{l} \mathcal{T}_{l}(\tau, \eta)
\end{array}\right),
\end{aligned}
$$

where

$$
S_{r}(\tau, \eta)\left(\tau \mathcal{A}_{0}+i \eta \mathcal{A}_{1}\right)_{r} \mathcal{T}_{r}(\tau, \eta)=\left(\begin{array}{cccr}
\tau+i v_{r} \eta & 0 & 0 & i c_{r}^{2} \eta \\
0 & \frac{1}{4}\left(\tau+i v_{r} \eta\right) & 0 & \frac{1}{2} i c_{r}^{2} \eta \\
0 & -\frac{1}{2} i c_{r}^{2} \eta & c_{r}^{4} \eta^{2} \omega_{r} & 0 \\
0 & 0 & 0 & \omega_{r}
\end{array}\right)
$$

and

$$
S_{l}(\tau, \eta)\left(\tau \mathcal{A}_{0}+i \eta \mathcal{A}_{1}\right)_{l} \mathcal{T}_{l}(\tau, \eta)=\left(\begin{array}{cccc}
\tau+i v_{l} \eta & 0 & 0 & 0 \\
0 & \frac{1}{4}\left(\tau+i v_{l} \eta\right) & 0 & 0 \\
0 & 0 & \omega_{l} & 0 \\
0 & 0 & 0 & \omega_{l}
\end{array}\right)
$$

Thus, we simultaneously "almost diagonalize" the original system of algebraicdifferential equations (4.22) due to (4.25). This is sufficient to derive energy estimates in such a neighborhood $\mathcal{V}$ of the pole $\left(\tau_{0}, \eta_{0}\right)$.

4.4. Derivation of the energy estimate. We now derive the estimates (3.21) and (3.22). Thanks to (4.4), (4.5), and (4.11), it is sufficient to estimate the trace of $W^{n c}$ on $\left\{x_{2}=0\right\}$.

As in $[11,44]$, the previous analysis shows that, for all $\left(\tau_{0}, \eta_{0}\right) \in \Sigma$, there exists a neighborhood $\mathcal{V}$ of $\left(\tau_{0}, \eta_{0}\right)$ in $\Sigma$ with desired properties. Because $\Sigma$ is a $C^{\infty}$ compact manifold, there exists a finite covering $\left(\mathcal{V}_{1}, \cdots, \mathcal{V}_{I}\right)$ of $\Sigma$ by such neighborhoods, and a smooth partition of unity $\left(\chi_{1}, \cdots, \chi_{I}\right)$ associated with this covering, that is, the functions $\chi_{1}, \cdots, \chi_{I}$ are nonnegative, $C^{\infty}$, and satisfy

$$
\operatorname{supp} \chi_{i} \subset \mathcal{V}_{i} \text { and } \sum_{i=1}^{I} \chi_{i}^{2} \equiv 1 \text {. }
$$

For $\left(\tau_{0}, \eta_{0}\right)$ belonging to the classes $(\mathrm{C} 1),(\mathrm{C} 2)$, and $(\mathrm{C} 4)$, the energy estimate can be obtained in the same way as in [11]. For $\left(\tau_{0}, \eta_{0}\right)$ belonging to the class $(\mathrm{C} 5)$, the energy estimate can be obtained similarly to [11] by employing "almost diagonal" matrices (4.25). More precisely, we have

$$
\lambda \chi_{i}^{2}(\tau, \eta) \int_{0}^{+\infty}\left|\widehat{W^{n c}}\left(\delta, \eta, x_{2}\right)\right|^{2} d x_{2}+\chi_{i}^{2}(\tau, \eta)\left|\widehat{W^{n c}}(\delta, \eta, 0)\right|^{2} \leq C_{i} \chi_{i}^{2}(\tau, \eta)|\hat{h}|^{2} .
$$

For $\left(\tau_{0}, \eta_{0}\right)$ belonging to the class $(\mathrm{C} 3)$, the energy estimate can be obtained as in [44] by using the estimate (4.21) as the following:

$$
\begin{aligned}
& \lambda \chi_{i}^{2}(\tau, \eta) \int_{0}^{+\infty}\left|\widehat{W^{n c}}\left(\delta, \eta, x_{2}\right)\right|^{2} d x_{2}+\chi_{i}^{2}(\tau, \eta)\left|\widehat{W^{n c}}(\delta, \eta, 0)\right|^{2} \\
\leq & \frac{C_{i}}{\lambda^{2 \nu_{0}}} \chi_{i}^{2}(\tau, \eta)|\hat{h}|^{2}\left(|\tau|^{2}+v_{r}^{2} \eta^{2}\right)^{2 \nu_{0}} .
\end{aligned}
$$


Adding up the above two estimates, using the partition of unity, then integrating the resulting inequality with respect to $(\delta, \eta) \in \mathbb{R}^{2}$, and employing Plancherel's theorem yield the desired estimate:

$$
\left\|\left|W^{n c}\right|_{x_{2}=0}\left|\left\|_{0}^{2}+\right\| W^{n c}\right|_{x_{2}=0}\right\|_{0}^{2} \leq \frac{C}{\lambda^{2 \nu_{0}}}\|g\|_{\nu_{0}, \lambda}^{2} .
$$

For $\nu_{0}=1$ or $\nu_{0}=2$ corresponding to $v_{r} \neq \frac{c_{r}+c_{l}}{2}$ or $v_{r}=\frac{c_{r}+c_{l}}{2}$, respectively, the combination of (4.4), (4.5), and (4.11) with (4.26) leads to the estimates (3.21) and (3.22). This completes the proof of Theorem 3.2 (equivalent to Theorem 3.1).

\section{Linear stability: variable coefficients}

For the case of variable coefficients, we first present the linearized problem and state the main result in this section, and then we prove the main result in the next section.

5.1. The linearized equations. We first introduce the linearized equations around a state $U_{r, l}\left(t, x_{1}, x_{2}\right), \Phi_{r, l}\left(t, x_{1}, x_{2}\right)$ that are given by a perturbation of the constant solution in (2.22). More precisely, let us consider the functions

$$
\begin{aligned}
& U_{r, l}\left(t, x_{1}, x_{2}\right)=\left(\begin{array}{c}
\bar{m}_{r, l} \\
\bar{n}_{r, l} \\
\pm \bar{v}_{r} \\
0
\end{array}\right)+\dot{U}_{r, l}\left(t, x_{1}, x_{2}\right), \\
& \Phi_{r, l}\left(t, x_{1}, x_{2}\right)= \pm x_{2}+\dot{\Phi}_{r, l}\left(t, x_{1}, x_{2}\right),
\end{aligned}
$$

where $\bar{m}_{r, l}, \bar{n}_{r, l}, \bar{v}_{r}$ are fixed positive constants and

$$
U_{r, l}\left(t, x_{1}, x_{2}\right) \equiv\left(\begin{array}{c}
m_{r, l} \\
n_{r, l} \\
v_{r, l} \\
u_{r, l}
\end{array}\right)\left(t, x_{1}, x_{2}\right), \quad \dot{U}_{r, l}\left(t, x_{1}, x_{2}\right) \equiv\left(\begin{array}{c}
\dot{m}_{r, l} \\
\dot{n}_{r, l} \\
\dot{v}_{r, l} \\
\dot{u}_{r, l}
\end{array}\right)\left(t, x_{1}, x_{2}\right) .
$$

The index $r$ (resp. $l$ ) denotes the state on the right (resp. on the left) of the interface (after the change of variables). Notice that $v_{r}\left(t, x_{1}, 0\right) \neq-v_{l}\left(t, x_{1}, 0\right)$ here. We assume that

$$
\begin{aligned}
& U_{r, l}, \quad \nabla \Phi_{r, l} \in W^{2, \infty}(\Omega), \\
& \left\|\left(U_{r}, U_{l}\right)\right\|_{W^{2, \infty}(\Omega)}+\left\|\left(\nabla \Phi_{r}, \nabla \Phi_{l}\right)\right\|_{W^{2, \infty}(\Omega)} \leq K_{0},
\end{aligned}
$$

where $K_{0}>0$ is constant and the perturbations $\dot{U}_{r, l}$ have compact support. The corresponding Rankine-Hugoniot conditions and the continuity condition for the functions $\Phi_{r, l}$ can be written in the form of (2.17) by dropping the $\sharp$ index as

$$
\begin{aligned}
& \left.\Phi_{r}\left(t, x_{1}, x_{2}\right)\right|_{x_{2}=0}=\left.\Phi_{l}\left(t, x_{1}, x_{2}\right)\right|_{x_{2}=0}=\varphi\left(t, x_{1}\right), \\
& \left.\left(v_{r}-v_{l}\right)\left(t, x_{1}, x_{2}\right)\right|_{x_{2}=0} \partial_{1} \varphi\left(t, x_{1}\right)-\left.\left(u_{r}-u_{l}\right)\left(t, x_{1}, x_{2}\right)\right|_{x_{2}=0}=0, \\
& \partial_{t} \varphi\left(t, x_{1}\right)+\left.v_{r}\left(t, x_{1}, x_{2}\right)\right|_{x_{2}=0} \partial_{1} \varphi\left(t, x_{1}\right)-\left.u_{r}\left(t, x_{1}, x_{2}\right)\right|_{x_{2}=0}=0, \\
& \left.\left(m_{r}+n_{r}\right)\left(t, x_{1}, x_{2}\right)\right|_{x_{2}=0}-\left.\left(m_{l}+n_{l}\right)\left(t, x_{1}, x_{2}\right)\right|_{x_{2}=0}=0 .
\end{aligned}
$$

The functions $\Phi_{r}$ and $\Phi_{l}$ should also satisfy the eikonal equations

$$
\begin{aligned}
& \partial_{t} \Phi_{r}+v_{r} \partial_{1} \Phi_{r}-u_{r}=0, \\
& \partial_{t} \Phi_{l}+v_{l} \partial_{1} \Phi_{l}-u_{l}=0
\end{aligned}
$$


together with

$$
\partial_{2} \Phi_{r}\left(t, x_{1}, x_{2}\right) \geq \kappa_{0}, \quad \partial_{2} \Phi_{l}\left(t, x_{1}, x_{2}\right) \leq-\kappa_{0}
$$

for a suitable constant $\kappa_{0}>0$ in the whole closed half-space $\left\{x_{2} \geq 0\right\}$.

Let us consider some families

$$
U_{s}^{ \pm}=U_{r, l}+s V^{ \pm}, \quad \Phi_{s}^{ \pm}=\Phi_{r, l}+s \Psi^{ \pm},
$$

where $s$ is a small parameter. We compute the linearized equations around the state $U_{r, l}, \Phi_{r, l}$ :

$$
L^{\prime}\left(U_{r, l}, \Phi_{r, l}\right)\left(V_{ \pm}, \Psi_{ \pm}\right):=\left.\left\{\frac{d}{d s} L\left(U_{s}^{ \pm}, \Phi_{s}^{ \pm}\right) U_{s}^{ \pm}\right\}\right|_{s=0}=f_{ \pm}
$$

We obtain

$$
\begin{aligned}
& L^{\prime}\left(U_{r}, \Phi_{r}\right)\left(V_{+}, \Psi_{+}\right) \\
= & \partial_{t} V_{+}+A_{1}\left(U_{r}\right) \partial_{1} V_{+}+\frac{1}{\partial_{2} \Phi_{r}}\left(A_{2}\left(U_{r}\right)-\partial_{t} \Phi_{r} I_{4 \times 4}-\partial_{1} \Phi_{r} A_{1}\left(U_{r}\right)\right) \partial_{2} V_{+} \\
& +\left[d A_{1}\left(U_{r}\right)\right] V_{+} \partial_{1} U_{r}-\frac{\partial_{2} \Psi_{+}}{\left(\partial_{2} \Phi_{r}\right)^{2}}\left[A_{2}\left(U_{r}\right)-\partial_{t} \Phi_{r} I_{4 \times 4}-\partial_{1} \Phi_{r} A_{1}\left(U_{r}\right)\right] \partial_{2} U_{r} \\
& \frac{1}{\partial_{2} \Phi_{r}}\left\{d\left[A_{2}\left(U_{r}\right)\right] V_{+}-\partial_{t} \Psi_{+} I_{4 \times 4}-\partial_{1} \Psi_{+} A_{1}\left(U_{r}\right)-\partial_{1} \Phi_{r} d\left[A_{1}\left(U_{r}\right)\right] V_{+}\right\} \partial_{2} U_{r} \\
= & f_{+}
\end{aligned}
$$

in the domain $\left\{x_{2}>0\right\}$, and we also obtain a similar equation for $L^{\prime}\left(U_{l}, \Phi_{l}\right)\left(V_{-}, \Psi_{-}\right)$ with $V_{-}, \Psi_{-}, U_{l}, \Phi_{l}, f_{-}$replacing $V_{+}, \Psi_{+}, U_{r}, \Phi_{r}, f_{+}$.

Recall that, according to the definition in (2.19) and (2.20), the second row in (5.7) may be simply denoted by

$$
\begin{aligned}
L\left(U_{r}, \nabla \Phi_{r}\right) V_{+}:= & \partial_{t} V_{+}+A_{1}\left(U_{r}\right) \partial_{1} V_{+} \\
& +\frac{1}{\partial_{2} \Phi_{r}}\left[A_{2}\left(U_{r}\right)-\partial_{t} \Phi_{r} I_{4 \times 4}-\partial_{1} \Phi_{r} A_{1}\left(U_{r}\right)\right] \partial_{2} V_{+}
\end{aligned}
$$

The linearized equation (5.7) and the corresponding one for $\left(V_{-}, \Psi_{-}\right)$may be simplified by introducing the "good unknown" as in [1]:

$$
\dot{V}_{+}=V_{+}-\frac{\Psi_{+}}{\partial_{2} \Phi_{r}} \partial_{2} U_{r}, \quad \dot{V}_{-}=V_{-}-\frac{\Psi_{-}}{\partial_{2} \Phi_{l}} \partial_{2} U_{l}
$$

A direct calculation shows that $\dot{V}_{+}$and $\dot{V}_{-}$satisfy

$$
\begin{aligned}
& L\left(U_{r}, \nabla \Phi_{r}\right) \dot{V}_{+}+C\left(U_{r}, \nabla U_{r}, \nabla \Phi_{r}\right) \dot{V}_{+}+\frac{\Psi_{+}}{\partial x_{2} \Phi_{r}} \partial_{2}\left\{L\left(U_{r}, \nabla \Phi_{r}\right) U_{r}\right\}=f_{+}, \\
& L\left(U_{l}, \nabla \Phi_{l}\right) \dot{V}_{-}+C\left(U_{l}, \nabla U_{l}, \nabla \Phi_{l}\right) \dot{V}_{-}+\frac{\Psi_{-}}{\partial x_{2} \Phi_{l}} \partial_{2}\left\{L\left(U_{l}, \nabla \Phi_{l}\right) U_{l}\right\}=f_{-}
\end{aligned}
$$

where

$$
\begin{aligned}
C\left(U_{r}, \nabla U_{r}, \nabla \Phi_{r}\right) \dot{V}_{+}:= & \left(d A_{1}\left(U_{r}\right) \dot{V}_{+}\right) \partial_{1} U_{r} \\
& +\frac{1}{\partial_{2} \Phi_{r}}\left\{d A_{2}\left(U_{r}\right) \dot{V}_{+}-\partial_{1} \Phi_{r}\left[d A_{1}\left(U_{r}\right) \dot{V}_{+}\right]\right\} \partial_{2} U_{r}
\end{aligned}
$$

with a similar expression for $C\left(U_{l}, \nabla U_{l}, \nabla \Phi_{l}\right) \dot{V}_{-}$. 
5.2. The effective linearized equations. From $[1,22]$, we neglect the zeroth order term $\Psi_{+}, \Psi_{-}$in the linearized equation (5.9) and consider the effective linear operators

$$
\begin{aligned}
& L_{r}^{\prime} \dot{V}_{+}:=L\left(U_{r}, \nabla \Phi_{r}\right) \dot{V}_{+}+C\left(U_{r}, \nabla U_{r}, \nabla \Phi_{r}\right) \dot{V}_{+}=f_{+} \\
& L_{l}^{\prime} \dot{V}_{-}:=L\left(U_{l}, \nabla \Phi_{l}\right) \dot{V}_{-}+C\left(U_{l}, \nabla U_{l}, \nabla \Phi_{l}\right) \dot{V}_{-}=f_{-} .
\end{aligned}
$$

We can easily verify, using (5.2), that the coefficients of the operators $L\left(U_{r}, \nabla \Phi_{r}\right)$ and $L\left(U_{l}, \nabla \Phi_{l}\right)$ are in $W^{2, \infty}(\Omega)$, that is,

$$
\begin{array}{ll}
A_{1}\left(U_{r}\right) \in W^{2, \infty}(\Omega), & \frac{1}{\partial_{2} \Phi_{r}}\left[A_{2}\left(U_{r}\right)-\partial_{t} \Phi_{r} I_{4 \times 4}-\partial_{1} \Phi_{r} A_{1}\left(U_{r}\right)\right] \in W^{2, \infty}(\Omega), \\
A_{1}\left(U_{l}\right) \in W^{2, \infty}(\Omega), & \frac{1}{\partial_{2} \Phi_{l}}\left[A_{2}\left(U_{l}\right)-\partial_{t} \Phi_{l} I_{4 \times 4}-\partial_{1} \Phi_{l} A_{1}\left(U_{l}\right)\right] \in W^{2, \infty}(\Omega) .
\end{array}
$$

Moreover, we have $C\left(U_{r, l}, \nabla U_{r, l}, \nabla \Phi_{r, l}\right) \in W^{1, \infty}(\Omega)$.

We note that the linearized equation (5.11) forms a symmetrizable hyperbolic system. As an example, a Friedrichs symmetrizer for the operator $L_{r, l}^{\prime}$ is

$$
S_{r, l}(t, x)=\left(\begin{array}{cccr}
\frac{p_{n}}{m_{r, l}} & 0 & 0 & 0 \\
0 & \frac{p_{n}}{n_{r, l}} & 0 & 0 \\
0 & 0 & n_{r, l} & 0 \\
0 & 0 & 0 & n_{r, l}
\end{array}\right)(t, x)
$$

Using the eikonal equation (5.4), we find (recall that $A_{1}(U), A_{2}(U)$ are defined by $(2.1)$ )

$$
\begin{aligned}
& \frac{S_{r}}{\partial_{2} \Phi_{r}}\left[A_{2}\left(U_{r}\right)-\partial_{t} \Phi_{r} I_{4 \times 4}-\partial_{1} \Phi_{r} A_{1}\left(U_{r}\right)\right] \\
= & \frac{1}{\partial_{2} \Phi_{r}}\left(\begin{array}{lccc}
0 & 0 & -p_{n} \partial_{1} \Phi_{r} & p_{n} \\
0 & 0 & -p_{n} \partial_{1} \Phi_{r} & p_{n} \\
-p_{n} \partial_{1} \Phi_{r} & -p_{n} \partial_{1} \Phi_{r} & 0 & 0 \\
p_{n} & p_{n} & 0 & 0
\end{array}\right),
\end{aligned}
$$

and thus expect to control the traces of the components $\dot{V}_{+, 1}+\dot{V}_{+, 2}$, and $\left(\dot{V}_{+, 4}-\partial_{1} \Phi_{r} \dot{V}_{+, 3}\right)$ on the boundary $\left\{x_{2}=0\right\}$. In the same way, we expect to control the traces of the components $\dot{V}_{-, 1}+\dot{V}_{-, 2}$, and $\left(\dot{V}_{-, 4}-\partial_{1} \Phi_{r, l} \dot{V}_{-, 3}\right)$ on the boundary. These preliminary considerations motivate the introduction of the following trace operator on the boundary:

$$
\left.\mathbb{P}(\varphi) \dot{V}_{ \pm}\right|_{x_{2}=0}:=\left(\begin{array}{c}
\dot{V}_{ \pm, 1}+\dot{V}_{ \pm, 2} \\
\dot{V}_{ \pm, 4}-\partial_{1} \Phi_{r, l} \dot{V}_{ \pm, 3}
\end{array}\right)_{x_{2}=0}
$$

This operator will be used in the energy estimates for the linearized equations.

REMARK 5.1. As in [44], one can check that the rows of (5.13) are just the traces of the non-characteristic components of $\dot{V}_{ \pm}$after multiplication of equation (5.11) by the symmetrizer $S_{r, l}$. 
5.3. The linearized boundary conditions. We now turn to the linearized boundary conditions. The linearization of (5.3) gives

$$
\begin{aligned}
& \left.\Psi^{+}\left(t, x_{1}, x_{2}\right)\right|_{x_{2}=0}=\left.\Psi^{-}\left(t, x_{1}, x_{2}\right)\right|_{x_{2}=0}=\psi\left(t, x_{1}\right), \\
& \left.\left(v_{r}-v_{l}\right)\right|_{x_{2}=0} \partial_{1} \psi+\left.\left(v_{+}-v_{-}\right)\right|_{x_{2}=0} \partial_{1} \varphi-\left.\left(u_{+}-u_{-}\right)\right|_{x_{2}=0}=g_{1}, \\
& \partial_{t} \psi+\left.v_{r}\right|_{x_{2}=0} \partial_{1} \psi+\left.v_{+}\right|_{x_{2}=0} \partial_{1} \varphi-\left.u_{+}\right|_{x_{2}=0}=g_{2}, \\
& \left.\left(m_{+}+n_{+}\right)\right|_{x_{2}=0}-\left.\left(m_{-}+n_{-}\right)\right|_{x_{2}=0}=g_{3}
\end{aligned}
$$

on the boundary $\left\{x_{2}=0\right\}$.

Let us introduce the matrices

$$
\begin{aligned}
& b\left(t, x_{1}\right)=\left(\begin{array}{ccc|c}
0 & \left.\left(v_{r}-v_{l}\right)\right|_{x_{2}=0} \\
1 & & \left.v_{r}\right|_{x_{2}=0}
\end{array}\right), \\
& 0\left(t, x_{1}\right)=\left(\begin{array}{ccccccccc}
0 & 0 & \partial_{1} \varphi & -1 & 0 & 0 & -\partial_{1} \varphi & 1 \\
0 & 0 & \partial_{1} \varphi & -1 & 0 & 0 & 0 & 0 \\
1 & 1 & 0 & 0 & -1 & -1 & 0 & 0
\end{array}\right) .
\end{aligned}
$$

Denote

$$
\begin{gathered}
V=\left(V_{+}, V_{-}\right)^{\top}=\left(m_{+}, n_{+}, v_{+}, u_{+}, m_{-}, n_{-}, v_{-}, u_{-}\right)^{\top}, \\
\nabla \psi=\left(\partial_{t} \psi, \partial_{1} \psi\right)^{\top}, \quad g=\left(g_{1}, g_{2}, g_{3}\right)^{\top} .
\end{gathered}
$$

Then, the linearized boundary conditions become equivalently

$$
\begin{aligned}
& \left.\Psi^{+}\left(t, x_{1}, x_{2}\right)\right|_{x_{2}=0}=\left.\Psi^{-}\left(t, x_{1}, x_{2}\right)\right|_{x_{2}=0}=\psi\left(t, x_{1}\right), \\
& b \nabla \psi+\left.M V\right|_{x_{2}=0}=g .
\end{aligned}
$$

In terms of the good unknown $\dot{V}=\left(\dot{V}_{+}, \dot{V}_{-}\right)^{\top}$ defined by (5.8), the linearized boundary conditions read as

$$
\begin{aligned}
& \left.\Psi^{+}\left(t, x_{1}, x_{2}\right)\right|_{x_{2}=0}=\left.\Psi^{-}\left(t, x_{1}, x_{2}\right)\right|_{x_{2}=0}=\psi\left(t, x_{1}\right), \\
& B^{\prime}\left(U_{r, l}, \Phi_{r, l}\right)\left(\left.\dot{V}\right|_{x_{2}=0}, \psi\right) \\
:= & b \nabla \psi+\left.M\left(\dot{V}_{+}+\frac{\Psi_{+}}{\partial_{2} \Phi_{r}} \partial_{2} U_{r}, \dot{V}_{-}+\frac{\Psi_{-}}{\partial_{2} \Phi_{l}} \partial_{2} U_{l}\right)^{\top}\right|_{x_{2}=0} \\
= & b \nabla \psi+\left.M\left(\frac{\partial_{2} U_{r}}{\partial_{2} \Phi_{r}} \psi, \frac{\partial_{2} U_{l}}{\partial_{2} \Phi_{l}} \psi\right)^{\top}\right|_{x_{2}=0}+\left.M \dot{V}\right|_{x_{2}=0} \\
= & b \nabla \psi+\left.\underbrace{\left.M\left(\begin{array}{l}
\frac{\partial_{2} U_{r}}{\partial_{2} \Phi_{r}} \\
\frac{\partial_{2} U_{l}}{\partial_{2} \Phi_{l}}
\end{array}\right)\right|_{x_{2}=0}}_{b_{\sharp}} \psi M \dot{V}\right|_{x_{2}=0}=g .
\end{aligned}
$$


5.4. Main result. We observe that, from (5.15), the linearized boundary conditions only involve $\left.\mathbb{P} \dot{V}_{ \pm}\right|_{x_{2}=0}$, where $\mathbb{P}$ is defined by (5.13). With this notation, we can state our main result (the norms are the weighted norms defined in Section 3) as follows.

TheOrem 5.1. Assume that the particular solution defined by (5.1) satisfies

$$
\bar{v}_{r}-\bar{v}_{l}>\left(\bar{c}_{r}^{\frac{2}{3}}+\bar{c}_{l}^{\frac{2}{3}}\right)^{\frac{3}{2}}, \quad \bar{v}_{r}-\bar{v}_{l} \neq \sqrt{2}\left(\bar{c}_{r}+\bar{c}_{l}\right)
$$

and that the perturbations $\dot{U}_{r, l}, \nabla \dot{\Phi}_{r, l}$ have compact support and are small enough in $W^{2, \infty}(\Omega)$. Then, there exist some constants $C_{1}$ and $\lambda_{1} \geq 1$ depending on $K_{0}$ and $\kappa_{0}$ (defined in (5.2) and (5.5)) such that, for all $\lambda \geq \lambda_{1}$, the solution $(\dot{V}, \psi) \in H_{\lambda}^{2}(\Omega) \times$ $H_{\lambda}^{2}\left(\mathbb{R}^{2}\right)$ to the linearized problem (5.11) and (5.16) satisfies the following estimates:

$$
\begin{aligned}
& \lambda\left\|\left|\dot{V}\left\|\left.\right|_{L_{\lambda}^{2}(\Omega)} ^{2}+\right\| \mathbb{P} \dot{V}\right|_{x_{2}=0}\right\|_{L_{\lambda}^{2}\left(\mathbb{R}^{2}\right)}^{2}+\|\psi\|_{H_{\lambda}^{1}\left(\mathbb{R}^{2}\right)}^{2} \\
\leq & C_{1}\left(\left.\frac{1}{\lambda^{3}}\left\|\left|L^{\prime} \dot{V}\left\|\left.\right|_{L^{2}\left(H_{\lambda}^{1}\right)} ^{2}+\frac{1}{\lambda^{2}}\right\|\right| B^{\prime}(\dot{V}, \psi)\right\|\right|_{H_{\lambda}^{1}\left(\mathbb{R}^{2}\right)} ^{2}\right) \\
:= & C_{1}\left(\frac { 1 } { \lambda ^ { 3 } } \left\|\left|\left(f_{+}, f_{-}\right)\left\|\left.\right|_{L^{2}\left(H_{\lambda}^{1}\right)} ^{2}+\frac{1}{\lambda^{2}}\right\| g\|\|_{H_{\lambda}^{1}\left(\mathbb{R}^{2}\right)}^{2}\right),\right.\right.
\end{aligned}
$$

where the linearized operators $L^{\prime}$ and $B^{\prime}$ are defined in (5.11) and (5.16).

REMARK 5.2. Theorem 5.1 is counterpart of Theorem 3.1 for variable coefficients.

\section{Proof of Theorem 5.1}

This section is devoted to the proof of Theorem 5.1 on the linear stability with variable coefficients.

\subsection{Some preliminary transformations.}

6.1.1. Preliminary transformations of the interior equations. For the linearized equation (5.11), from multiplication by the Friedrichs symmetrizer defined in the previous section and an integration by parts, one has the following lemma.

Lemma 6.1. There exist two constants $C>0$ and $\lambda_{0} \geq 1$ such that, for all $\lambda \geq \lambda_{0}$, the following estimates hold:

$$
\left.\lambda\left\|\left|\dot{V}_{ \pm}\left\|\left.\right|_{L_{\lambda}^{2}(\Omega)} ^{2} \leq \frac{C}{\lambda}\right\|\right| L_{r}^{\prime} \dot{V}_{ \pm}\right\|\right|_{L_{\lambda}^{2}(\Omega)} ^{2}+\left\|\left.\mathbb{P} \dot{V}_{ \pm}\right|_{x_{2}=0}\right\|_{L_{\lambda}^{2}\left(\mathbb{R}^{2}\right)}^{2}
$$

and thus

$$
\left.\lambda\left\|\left|\dot{V}\left\|\left.\right|_{L_{\lambda}^{2}(\Omega)} ^{2} \leq \frac{C}{\lambda}\right\|\right| L^{\prime} \dot{V}\right\|\right|_{L_{\lambda}^{2}(\Omega)} ^{2}+\left\|\left.\mathbb{P} \dot{V}\right|_{x_{2}=0}\right\|_{L_{\lambda}^{2}\left(\mathbb{R}^{2}\right)}^{2},
$$

where the operator $\mathbb{P}$ is defined in (5.13).

As in the case of constant coefficients, we only need estimate the traces $\left.\mathbb{P} \dot{V}_{ \pm}\right|_{x_{2}=0}$ and the front function $\psi$ in terms of the source terms in the interior domain and on the boundary. For this purpose, we shall reformulate further the interior equation (5.11) to deal with the matrix coefficient of $\partial_{2}$ in the differential operators $L_{r, l}^{\prime}$, noticing that the boundary matrix has constant rank in the whole closed half-space. Thus, we first consider the coefficients of $\partial_{2} \dot{V}_{ \pm}$in (5.11) which are equal to

$$
\frac{1}{\partial_{2} \Phi}\left[A_{2}(U)-\partial_{t} \Phi I_{4 \times 4}-\partial_{1} \Phi A_{1}(U)\right]
$$


where we drop the indices $r, l$. Under assumption (5.4), (6.1) reduces to the matrix

$$
\begin{aligned}
\bar{A}_{2}(U, \nabla \Phi) & =\frac{1}{\partial_{2} \Phi}\left[A_{2}(U)-\partial_{t} \Phi I_{4 \times 4}-\partial_{1} \Phi A_{1}(U)\right] \\
& =\frac{1}{\partial_{2} \Phi}\left(\begin{array}{lccc}
0 & 0 & -m \partial_{1} \Phi & m \\
0 & 0 & -n \partial_{1} \Phi & n \\
-\frac{p_{n} \partial_{1} \Phi}{n} & -\frac{p_{n} \partial_{1} \Phi}{n} & 0 & 0 \\
\frac{p_{n}}{n} & \frac{p_{n}}{n} & 0 & 0
\end{array}\right),
\end{aligned}
$$

which has eigenvalues

$$
\lambda_{1}^{*}=0 \quad \text { with multiplicity } 2, \quad \text { and } \quad \lambda_{2,3}^{*}= \pm \frac{c(m, n)\left\langle\partial_{1} \Phi\right\rangle}{\partial_{2} \Phi} .
$$

Here, $c(m, n)$ is defined by $(3.7)$ and we denote $\left\langle\partial_{1} \Phi\right\rangle:=\sqrt{1+\left(\partial_{1} \Phi\right)^{2}}$.

We now diagonalize the above matrix. The eigenvectors associated with the eigenvalues are

$$
\begin{aligned}
& (1,-1,0,0)^{\top}, \quad\left(0,0,1, \partial_{1} \Phi\right)^{\top}, \quad \text { for } \lambda_{1}^{*}, \\
& \left(\frac{m}{n}\left\langle\partial_{1} \Phi\right\rangle,\left\langle\partial_{1} \Phi\right\rangle,-\frac{c(m, n)}{n} \partial_{1} \Phi, \frac{c(m, n)}{n}\right)^{\top}, \text { for } \lambda_{2}^{*} \text {, } \\
& \left(\frac{m}{n}\left\langle\partial_{1} \Phi\right\rangle,\left\langle\partial_{1} \Phi\right\rangle, \frac{c(m, n)}{n} \partial_{1} \Phi,-\frac{c(m, n)}{n}\right)^{\top}, \text { for } \lambda_{3}^{*} \text {. }
\end{aligned}
$$

Observe that these eigenvectors are not orthonormal. Thus, we may define the following (non orthogonal) matrix

$$
T(U, \nabla \Phi):=\left(\begin{array}{cccr}
1 & 0 & \frac{m}{n}\left\langle\partial_{1} \Phi\right\rangle & \frac{m}{n}\left\langle\partial_{1} \Phi\right\rangle \\
-1 & 0 & \left\langle\partial_{1} \Phi\right\rangle & \left\langle\partial_{1} \Phi\right\rangle \\
0 & 1 & -\frac{c(m, n)}{n} \partial_{1} \Phi & \frac{c(m, n)}{n} \partial_{1} \Phi \\
0 & \partial_{1} \Phi & \frac{c(m, n)}{n} & -\frac{c(m, n)}{n}
\end{array}\right) .
$$

Then, by direct calculations, the inverse $T^{-1}(U, \nabla \Phi)$ is

$$
\left(\begin{array}{lccr}
\frac{n}{m+n} & -\frac{m}{m+n} & 0 & 0 \\
0 & 0 & \frac{1}{\left\langle\partial_{1} \Phi\right\rangle^{2}} & \frac{\partial_{1} \Phi}{\left\langle\partial_{1} \Phi\right\rangle^{2}} \\
\frac{n}{2(m+n)\left\langle\partial_{1} \Phi\right\rangle} & \frac{n}{2(m+n)\left\langle\partial_{1} \Phi\right\rangle} & -\frac{n}{2 c(m, n)} \frac{\partial_{1} \Phi}{\left\langle\partial_{1} \Phi\right\rangle^{2}} & \frac{n}{2 c(m, n)} \frac{1}{\left\langle\partial_{1} \Phi\right\rangle^{2}} \\
\frac{n}{2(m+n)\left\langle\partial_{1} \Phi\right\rangle} & \frac{n}{2(m+n)\left\langle\partial_{1} \Phi\right\rangle} & \frac{n}{2 c(m, n)} \frac{\partial_{1} \Phi}{\left\langle\partial_{1} \Phi\right\rangle^{2}} & -\frac{n}{2 c(m, n)} \frac{1}{\left\langle\partial_{1} \Phi\right\rangle^{2}}
\end{array}\right),
$$

which allows one to diagonalize the matrix $\bar{A}_{2}(U, \nabla \Phi)$ as

$$
T^{-1}(U, \nabla \Phi) \bar{A}_{2}(U, \nabla \Phi) T(U, \nabla \Phi)=\left(\begin{array}{cccc}
0 & 0 & 0 & 0 \\
0 & 0 & 0 & 0 \\
0 & 0 & \lambda_{2}^{*} & 0 \\
0 & 0 & 0 & \lambda_{3}^{*}
\end{array}\right)
$$

In order to obtain a constant boundary matrix in the differential operators, we also 
introduce the matrix

$$
\begin{aligned}
A_{0}(U, \nabla \Phi): & =\left(\begin{array}{cccr}
1 & 0 & 0 & 0 \\
0 & 1 & 0 & 0 \\
0 & 0 & \lambda_{2}^{*-1} & 0 \\
0 & 0 & 0 & \lambda_{3}^{*-1}
\end{array}\right) \\
& =\left(\begin{array}{cccc}
1 & 0 & 0 & 0 \\
0 & 1 & 0 & 0 \\
0 & 0 & \frac{\partial_{2} \Phi}{c(m, n)\left\langle\partial_{1} \Phi\right\rangle} & 0 \\
0 & 0 & 0 & -\frac{\partial_{2} \Phi}{c(m, n)\left\langle\partial_{1} \Phi\right\rangle}
\end{array}\right) .
\end{aligned}
$$

It follows that $A_{0} T^{-1} \bar{A}_{2} T=I_{2}:=\operatorname{diag}(0,0,1,1)$.

Let us define the new unknown functions

$$
W^{+}:=T^{-1}\left(U_{r}, \nabla \Phi_{r}\right) \dot{V}_{+}, \quad W^{-}:=T^{-1}\left(U_{l}, \nabla \Phi_{l}\right) \dot{V}_{-}
$$

and set

$$
T_{r, l}:=T\left(U_{r, l}, \nabla \Phi_{r, l}\right), \quad A_{0}^{r, l}:=A_{0}\left(U_{r, l}, \nabla \Phi_{r, l}\right) .
$$

By multiplying on the left of the equations in (5.11) by $A_{0}^{r, l} T_{r, l}^{-1}$, we see that $W^{ \pm}$solve the following equations (see Appendix A for details):

$$
\begin{aligned}
& A_{0}^{r} \partial_{t} W^{+}+A_{1}^{r} \partial_{1} W^{+}+I_{2} \partial_{2} W^{+}+A_{0}^{r} C^{r} W^{+}=F^{+}, \\
& A_{0}^{l} \partial_{t} W^{-}+A_{1}^{l} \partial_{1} W^{-}+I_{2} \partial_{2} W^{-}+A_{0}^{l} C^{l} W^{-}=F^{-},
\end{aligned}
$$

where we have set with slight abuse of notation

$$
\begin{aligned}
& A_{1}^{r, l}:=A_{0}^{r, l} T^{-1} A_{1} T\left(U_{r, l}, \nabla \Phi_{r, l}\right), \\
& C^{r, l}:=\left[T^{-1} \partial_{t} T+T^{-1} A_{1} \partial_{1} T+T^{-1} \bar{A}_{2} \partial_{2} T+T^{-1} C T\right]\left(U_{r, l}, \nabla U_{r, l}, \nabla \Phi_{r, l}\right), \\
& F^{ \pm}=A_{0}^{r, l} T_{r, l}^{-1} f_{ \pm},
\end{aligned}
$$

with

$$
A_{1}^{r}=\left(\begin{array}{cccr}
v_{r} & 0 & 0 & 0 \\
0 & v_{r} & \frac{c_{r}^{2}}{n_{r}\left\langle\partial_{1} \Phi_{r}\right\rangle} & \frac{c_{r}^{2}}{n_{r}\left\langle\partial_{1} \Phi_{r}\right\rangle} \\
0 & \frac{n_{r}}{2 c_{r}} \frac{\partial_{2} \Phi_{r}}{\left\langle\partial_{1} \Phi_{r}\right\rangle^{2}} & \left(\frac{v_{r}}{c_{r}}-\frac{\partial_{1} \Phi_{r}}{\left\langle\partial_{1} \Phi_{r}\right\rangle}\right) \frac{\partial_{2} \Phi_{r}}{\left\langle\partial_{1} \Phi_{r}\right\rangle} & 0 \\
0 & -\frac{n_{r}}{2 c_{r}} \frac{\partial_{2} \Phi_{r}}{\left\langle\partial_{1} \Phi_{r}\right\rangle^{2}} & 0 & -\left(\frac{v_{r}}{c_{r}}+\frac{\partial_{1} \Phi_{r}}{\left\langle\partial_{1} \Phi_{r}\right\rangle}\right) \frac{\partial_{2} \Phi_{r}}{\left\langle\partial_{1} \Phi_{r}\right\rangle}
\end{array}\right),
$$

The matrix $A_{1}^{l}$ is similar by changing index $r$ by $l$. Notice that the matrix coefficient of $\partial_{2}$ in (6.5) is the constant and diagonal boundary matrix $I_{2}$.

The above equations (6.5) are equivalent to the linearized equations (5.11). Introducing $\tilde{W}^{ \pm}=\mathrm{e}^{-\lambda t} W^{ \pm}$, one can rewrite equation (6.5) equivalently as

$$
\begin{aligned}
\mathcal{L}_{r}^{\lambda} \tilde{W}^{+}:= & \lambda A_{0}^{r} \tilde{W}^{+}+A_{0}^{r} \partial_{t} \tilde{W}^{+} \\
& +A_{1}^{r} \partial_{1} \tilde{W}^{+}+I_{2} \partial_{2} \tilde{W}^{+}+A_{0}^{r} C^{r} \tilde{W}^{+}=\mathrm{e}^{-\lambda t} F^{+}, \\
\mathcal{L}_{l}^{\lambda} \tilde{W}^{-}:= & \lambda A_{0}^{l} \tilde{W}^{-}+A_{0}^{l} \partial_{t} \tilde{W}^{-} \\
& +A_{1}^{l} \partial_{1} \tilde{W}^{-}+I_{2} \partial_{2} \tilde{W}^{-}+A_{0}^{l} C^{l} \tilde{W}^{-}=\mathrm{e}^{-\lambda t} F^{-} .
\end{aligned}
$$

Recall that we have $A_{j}^{r, l} \in W^{2, \infty}(\Omega)(j=0,1)$, and $C^{r, l} \in W^{1, \infty}(\Omega)$. 
6.1.2. Preliminary transformations of the boundary conditions. Denote the vector $W=\left(W^{+}, W^{-}\right)^{\top}=\left(T_{r}^{-1} \dot{V}_{+}, T_{l}^{-1} \dot{V}_{-}\right)^{\top}$. Then, the boundary conditions $(5.16)$ become

$$
\begin{aligned}
& \left.\Psi^{+}\left(t, x_{1}, x_{2}\right)\right|_{x_{2}=0}=\left.\Psi^{-}\left(t, x_{1}, x_{2}\right)\right|_{x_{2}=0}=\psi\left(t, x_{1}\right), \\
& b \nabla \psi+b_{\sharp} \psi+\left.M\left(\begin{array}{ll}
T_{r} 0 \\
0 & T_{l}
\end{array}\right)\left(\begin{array}{ll}
T_{r}^{-1} & 0 \\
0 & T_{l}^{-1}
\end{array}\right)\left(\begin{array}{l}
\dot{V}_{+} \\
\dot{V}_{-}
\end{array}\right)\right|_{x_{2}=0} \\
= & b \nabla \psi+b_{\sharp} \psi+\left.M\left(\begin{array}{cc}
T_{r} & 0 \\
0 & T_{l}
\end{array}\right) W\right|_{x_{2}=0}=g .
\end{aligned}
$$

Introducing $\tilde{W}^{ \pm}=\mathrm{e}^{-\lambda t} W^{ \pm}, \tilde{\Psi}_{ \pm}:=\mathrm{e}^{-\lambda t} \Psi, \tilde{\psi}_{ \pm}:=\mathrm{e}^{-\lambda t} \psi$ and $\mathbf{b}_{0}:=b(1,0)^{\top}=(0,1,0)^{\top}$, we obtain

$$
b \mathrm{e}^{-\lambda t} \nabla \psi=b \nabla\left(\mathrm{e}^{-\lambda t} \psi\right)+\lambda b \mathrm{e}^{-\lambda t} \psi \nabla t=b \nabla \tilde{\psi}+\lambda \tilde{\psi} b(1,0)^{\top}=b \nabla \tilde{\psi}+\lambda \mathbf{b}_{0} \tilde{\psi},
$$

Then, the equations (6.7) are also equivalent to

$$
\begin{aligned}
& \left.\tilde{\Psi}^{+}\left(t, x_{1}, x_{2}\right)\right|_{x_{2}=0}=\left.\tilde{\Psi}^{-}\left(t, x_{1}, x_{2}\right)\right|_{x_{2}=0}=\tilde{\psi}\left(t, x_{1}\right), \\
& \mathcal{B}^{\lambda}(\tilde{W}, \tilde{\psi}):=\lambda \mathbf{b}_{0} \tilde{\psi}+b \nabla \tilde{\psi}+b_{\sharp} \tilde{\psi}+\left.M\left(\begin{array}{cc}
T_{r} & 0 \\
0 & T_{l}
\end{array}\right) \tilde{W}\right|_{x_{2}=0}=\mathrm{e}^{-\lambda t} g .
\end{aligned}
$$

From (5.2) we have

$$
\begin{aligned}
& b \in W^{2, \infty}\left(\mathbb{R}^{2}\right), \quad b_{\sharp} \in W^{1, \infty}\left(\mathbb{R}^{2}\right), \\
& M \in W^{2, \infty}\left(\mathbb{R}^{2}\right),\left.\quad T_{r, l}\right|_{x_{2}=0} \in W^{2, \infty}\left(\mathbb{R}^{2}\right) .
\end{aligned}
$$

6.1.3. A priori estimate for the weighted linearized problem. We now derive an a priori estimate of the solution to the (weighted) linearized problem (6.6) and (6.8). By Lemma 6.1, we are looking for an estimate of $\mathbb{P} \dot{V}_{+}$and $\mathbb{P} \dot{V}_{-}$using the new function $W$. From the relations

$$
\begin{aligned}
& \left.\mathbb{P} \dot{V}_{+}\right|_{x_{2}=0}=\left(\begin{array}{c}
\left.\left(1+\frac{m_{r}}{n_{r}}\right)\left\langle\partial_{1} \varphi\right\rangle\left(W_{3}^{+}+W_{4}^{+}\right)\right|_{x_{2}=0} \\
\left.\frac{c_{r}}{n_{r}}\left\langle\partial_{1} \varphi\right\rangle^{2}\left(W_{3}^{+}-W_{4}^{+}\right)\right|_{x_{2}=0}
\end{array}\right), \\
& \left.\mathbb{P} \dot{V}_{-}\right|_{x_{2}=0}=\left(\begin{array}{c}
\left.\left(1+\frac{m_{l}}{n_{l}}\right)\left\langle\partial_{1} \varphi\right\rangle\left(W_{3}^{-}+W_{4}^{-}\right)\right|_{x_{2}=0} \\
\left.\frac{c_{l}}{n_{l}}\left\langle\partial_{1} \varphi\right\rangle^{2}\left(W_{3}^{-}-W_{4}^{-}\right)\right|_{x_{2}=0}
\end{array}\right),
\end{aligned}
$$

one has

$$
\begin{aligned}
&\left\|\left.\mathbb{P} \dot{V}_{+}\right|_{x_{2}=0}\right\|_{L_{\lambda}^{2}\left(\mathbb{R}^{2}\right)}+\left\|\left.\mathbb{P} \dot{V}_{-}\right|_{x_{2}=0}\right\|_{L_{\lambda}^{2}\left(\mathbb{R}^{2}\right)} \\
& \leq C\left(\left\|\left.\left(W_{3}^{+}, W_{4}^{+}\right)\right|_{x_{2}=0}\right\|_{L_{\lambda}^{2}\left(\mathbb{R}^{2}\right)}+\left\|\left.\left(W_{3}^{-}, W_{4}^{-}\right)\right|_{x_{2}=0}\right\|_{L_{\lambda}^{2}\left(\mathbb{R}^{2}\right)}\right) .
\end{aligned}
$$

We need to estimate the trace of the vector $\left(\tilde{W}_{3}^{+}, \tilde{W}_{4}^{+}, \tilde{W}_{3}^{-}, \tilde{W}_{4}^{-}\right)$for a solution $\tilde{W}$ to the (weighted) linearized equations (6.6) and (6.8). From now on, for the sake of simplicity, we drop the tildes and write $W^{ \pm}, \Psi_{ \pm}, \psi$ instead of $\tilde{W}^{ \pm}, \tilde{\Psi}_{ \pm}, \tilde{\psi}$. We note that $\Psi_{ \pm}, \psi$ are coupled with $W^{ \pm}$only through the boundary conditions. 
6.2. Paralinearization. We now apply paralinearization (see Bony [5] and Meyer [42]) to reduce the problem to the case of constant coefficients. The Fourier dual variables of $\left(t, x_{1}\right)$ are $(\delta, \eta)$. Denote $\tau=\lambda+i \delta$ the Laplace dual variable of $t$. We recall that the positive constants $K_{0}, \kappa_{0}$ were introduced in (5.2) and (5.5).

6.2.1. The boundary conditions. Define the following symbols:

$$
\begin{gathered}
\mathbf{b}_{0}:=\left(\begin{array}{l}
0 \\
1 \\
0
\end{array}\right), \\
\mathbf{b}_{1}\left(t, x_{1}\right):=\left(\begin{array}{c}
v_{r}-v_{l} \\
v_{r} \\
0
\end{array}\right)\left(t, x_{1}, 0\right), \\
\mathbf{b}\left(t, x_{1}, \delta, \eta, \lambda\right):=\tau \mathbf{b}_{0}+i \eta \mathbf{b}_{1}\left(t, x_{1}\right) .
\end{gathered}
$$

Because $\mathbf{b}_{0}$ is constant, we have

$$
\lambda \mathbf{b}_{0} \psi+\mathbf{b}_{0} \partial_{t} \psi=T_{\tau \mathbf{b}_{0}}^{\lambda} \psi
$$

The main paralinearization estimate yields

$$
\left\|\mathbf{b}_{1} \partial_{1} \psi-T_{i \eta \mathbf{b}_{1}}^{\lambda} \psi\right\|_{1, \lambda} \leq C\left\|\mathbf{b}_{1}\right\|_{W^{2, \infty}\left(\mathbb{R}^{2}\right)}\|\psi\|_{0} \leq \frac{C\left(K_{0}\right)}{\lambda}\|\psi\|_{1, \lambda} .
$$

We now easily obtain

$$
\left\|\lambda \mathbf{b}_{0} \psi+\mathbf{b}_{0} \partial_{t} \psi+\mathbf{b}_{1} \partial_{1} \psi-T_{\mathbf{b}}^{\lambda} \psi\right\|_{1, \lambda} \leq \frac{C\left(K_{0}\right)}{\lambda}\|\psi\|_{1, \lambda} .
$$

We also have the following inequalities:

$$
\begin{aligned}
& \left\|\mathbf{b}_{\sharp} \psi-T_{\mathbf{b}_{\sharp}}^{\lambda} \psi\right\|_{1, \lambda} \leq C\left\|\mathbf{b}_{\sharp}\right\|_{W^{1, \infty}\left(\mathbb{R}^{2}\right)}\|\psi\|_{0} \leq \frac{C\left(K_{0}, \kappa_{0}\right)}{\lambda}\|\psi\|_{1, \lambda}, \\
& \left\|T_{\mathbf{b}_{\sharp}}^{\lambda} \psi\right\|_{1, \lambda} \leq C\left\|\mathbf{b}_{\sharp}\right\|_{L^{\infty}\left(\mathbb{R}^{2}\right)}\|\psi\|_{1, \lambda} \leq C\left(K_{0}, \kappa_{0}\right)\|\psi\|_{1, \lambda},
\end{aligned}
$$

where $\mathbf{b}_{\sharp}$ is defined by (5.16). Finally, we define the symbol

$$
\mathbf{M}:=M\left(t, x_{1}, 0\right)\left(\begin{array}{cc}
T_{r} & 0 \\
0 & T_{l}
\end{array}\right)\left(t, x_{1}, 0\right),
$$

with the matrices $M, T_{r}, T_{l}$ defined in (5.15) and (6.2). Recall that the state around which the equations are linearized satisfies

$$
\Phi_{r}\left(t, x_{1}, 0\right)=\Phi_{l}\left(t, x_{1}, 0\right)=\varphi\left(t, x_{1}\right), \quad\left(n_{r}+m_{r}\right)\left(t, x_{1}, 0\right)=\left(n_{l}+m_{l}\right)\left(t, x_{1}, 0\right) .
$$

A direct calculation yields

$$
\mathbf{M}=\left(\begin{array}{ll}
M_{r} & M_{l}
\end{array}\right)\left(t, x_{1}, 0\right)\left(\begin{array}{cc}
T_{r} & 0 \\
0 & T_{l}
\end{array}\right)\left(t, x_{1}, 0\right)=\left(\begin{array}{ll}
\mathbf{M}_{r} & \mathbf{M}_{l}
\end{array}\right)\left(t, x_{1}, 0\right)
$$


Here,

and

$$
\begin{aligned}
& \left(\begin{array}{ll}
M_{r} & M_{l}
\end{array}\right)\left(t, x_{1}, 0\right)=: M\left(t, x_{1}\right)=\left(\begin{array}{cccccccc}
0 & 0 & \partial_{1} \varphi & -1 & 0 & 0 & -\partial_{1} \varphi & 1 \\
0 & 0 & \partial_{1} \varphi & -1 & 0 & 0 & 0 & 0 \\
1 & 1 & 0 & 0 & -1 & -1 & 0 & 0
\end{array}\right), \\
& \mathbf{M}_{r}=\left(\begin{array}{cccr}
0 & 0 & -\frac{c_{r}}{n_{r}}\left\langle\partial_{1} \varphi\right\rangle^{2} & \frac{c_{r}}{n_{r}}\left\langle\partial_{1} \varphi\right\rangle^{2} \\
0 & 0 & -\frac{c_{r}}{n_{r}}\left\langle\partial_{1} \varphi\right\rangle^{2} & \frac{c_{r}}{n_{r}}\left\langle\partial_{1} \varphi\right\rangle^{2} \\
0 & 0 & \left(1+\frac{m_{r}}{n_{r}}\right)\left\langle\partial_{1} \varphi\right\rangle & \left(1+\frac{m_{r}}{n_{r}}\right)\left\langle\partial_{1} \varphi\right\rangle
\end{array}\right)
\end{aligned}
$$

$$
\mathbf{M}_{l}=\left(\begin{array}{cccr}
0 & 0 & \frac{c_{l}}{n_{l}}\left\langle\partial_{1} \varphi\right\rangle^{2} & -\frac{c_{l}}{n_{l}}\left\langle\partial_{1} \varphi\right\rangle^{2} \\
0 & 0 & 0 & 0 \\
0 & 0 & -\left(1+\frac{m_{l}}{n_{l}}\right)\left\langle\partial_{1} \varphi\right\rangle & -\left(1+\frac{m_{l}}{n_{l}}\right)\left\langle\partial_{1} \varphi\right\rangle
\end{array}\right)
$$

Thus, the matrix $\mathbf{M}$ only acts on the noncharacteristic part of the vector $\left(W^{+}, W^{-}\right)$, that is, $W^{n c}=\left(W_{3}^{+}, W_{4}^{+}, W_{3}^{-}, W_{4}^{-}\right)$. Since $\mathbf{M} \in W^{2, \infty}\left(\mathbb{R}^{2}\right)$, from (6.14) and (6.15) we have

$$
\begin{aligned}
\left\|\left.\mathbf{M} W\right|_{x_{2}=0}-\left.T_{\mathbf{M}}^{\lambda} W\right|_{x_{2}=0}\right\|_{1, \lambda} & \leq \frac{C}{\lambda}\|\mathbf{M}\|_{W^{2, \infty}\left(\mathbb{R}^{2}\right)}\left\|\left.W^{n c}\right|_{x_{2}=0}\right\|_{0} \\
& \leq \frac{C\left(K_{0}\right)}{\lambda}\left\|\left.W\right|_{x_{2}=0} ^{n c}\right\|_{0} .
\end{aligned}
$$

Adding (6.14), (6.15), and (6.17), we obtain the paralinearization estimate for the boundary operator:

$$
\begin{gathered}
\left\|\mathcal{B}^{\lambda}(W, \psi)-T_{\mathbf{b}}^{\lambda} \psi-\left.T_{\mathbf{M}}^{\lambda} W\right|_{x_{2}=0}\right\|_{1, \lambda} \\
\leq C\left(K_{0}, \kappa_{0}\right)\left(\|\psi\|_{1, \lambda}+\frac{1}{\lambda}\left\|\left.W^{n c}\right|_{x_{2}=0}\right\|_{0}\right) .
\end{gathered}
$$

Recall that the boundary operator $\mathcal{B}^{\lambda}$ is defined by (6.8). Observe that, in the paralinearized version of $\mathcal{B}^{\lambda}$, there is no more zeroth order term in $\psi$.

6.2.2. The interior equations. We first estimate the paralinearization error for fixed $x_{2}$ and then integrate with respect to $x_{2}$. For instance, we have

$$
\begin{aligned}
& \left|\left\|\lambda \mathbf{A}_{0}^{r} W^{+}-T_{\lambda \mathbf{A}_{0}^{r}}^{\lambda} W^{+}\right\|\right|_{1, \lambda}^{2} \\
= & \int_{0}^{+\infty} \lambda^{2}\left\|\mathbf{A}_{0}^{r} W^{+}\left(\cdot, x_{2}\right)-T_{\mathbf{A}_{0}^{r}}^{\lambda} W^{+}\left(\cdot, x_{2}\right)\right\|_{1, \lambda}^{2} d x_{2} \\
\leq & C \int_{0}^{+\infty}\left\|\mathbf{A}_{0}^{r}\left(\cdot, x_{2}\right)\right\|_{W^{2, \infty}\left(\mathbb{R}^{2}\right)}^{2}\left\|W^{+}\left(\cdot, x_{2}\right)\right\|_{0}^{2} d x_{2} \\
\leq & C\left\|\mathbf{A}_{0}^{r}\right\|_{W^{2, \infty}\left(\mathbb{R}^{2}\right)}^{2}\left|\left\|W^{+}\right\|\right|_{0}^{2} \leq\left. C\left(K_{0}\right)\left\|W^{+}\right\|\right|_{0} ^{2} .
\end{aligned}
$$

Similarly, we have the following estimates:

$$
\begin{aligned}
& \left\|\left|\mathbf{A}_{0}^{r} \partial_{t} W^{+}-T_{i \delta \mathbf{A}_{0}^{r}}^{\lambda} W^{+}\left\|\left.\right|_{1, \lambda} \leq C\left(K_{0}\right) \mid\right\| W^{+}\|\|_{0},\right.\right. \\
& \left\|\left|\mathbf{A}_{1}^{r} \partial_{1} W^{+}-T_{i \eta \mathbf{A}_{1}^{r}}^{\lambda} W^{+}\left\|\left.\right|_{1, \lambda} \leq C\left(K_{0}\right) \mid\right\| W^{+}\|\|_{0},\right.\right. \\
& \left.\left\|\mathbf{A}_{0}^{r} \mathbf{C}^{r} W^{+}-T_{\mathbf{A}_{0}^{r} \mathbf{C}^{r}}^{\lambda} W^{+}\right\|\right|_{1, \lambda} \leq C\left(K_{0}, \kappa_{0}\right)\left|\left\|W^{+}\right\|\right|_{0} .
\end{aligned}
$$


Adding these inequalities, we end up with the paralinearization estimate for the interior equations:

$$
\left|\left\|\mathcal{L}_{r}^{\lambda} W^{+}-T_{\tau}^{\lambda} \mathbf{A}_{0}^{r}+i \eta \mathbf{A}_{1}^{r}+\mathbf{A}_{0}^{r} \mathbf{C}^{r} W^{+}-\mathbf{I}_{2} \partial_{2} W^{+}\right\|\right|_{1, \lambda} \leq C\left(K_{0}, \kappa_{0}\right)\left|\left\|W^{+}\right\|\right|_{0},
$$

where the linearized operator $\mathcal{L}_{r}^{\lambda}$ is defined by (6.6). The estimate for the equation on $W^{-}$is identical, that is,

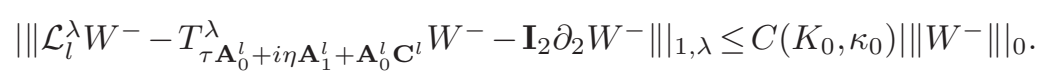

REMARK 6.1. In fact, both (6.19) and (6.20) give the error estimates between the differential operators $\mathcal{L}_{r, l}^{\lambda}$ and the paradifferential operators $T_{\tau \mathbf{A}_{0}^{r, l}+i \eta \mathbf{A}_{1}^{r, l}+\mathbf{A}_{0}^{r, l} \mathbf{C}^{r, l}}^{\lambda}+\mathbf{I}_{2} \partial_{2}$.

6.2.3. Elimination of the front. As in the case of constant coefficients we shall eliminate the front $\psi$ in the (paralinearized) boundary conditions. If the perturbation is small enough (in the $L^{\infty}$ norm), there exists a constant $c>0$ (depending only on $K_{0}$ ) such that

$$
\left|\mathbf{b}\left(t, x_{1}, \delta, \eta, \lambda\right)\right|^{2} \geq c\left(\lambda^{2}+\delta^{2}+\eta^{2}\right) .
$$

Applying Garding's inequality, we obtain

$$
\mathfrak{R}\left\langle T_{\mathbf{b}^{*} \mathbf{b}}^{\lambda} \psi, \psi\right\rangle_{L^{2}\left(\mathbb{R}^{2}\right)} \geq \frac{c}{2}\|\psi\|_{1, \lambda}^{2}
$$

for all $\lambda \geq \lambda_{0}$ (where $\lambda_{0}$ only depends on $K_{0}$ ). Using the rules of symbolic calculus, we have $T_{\mathbf{b}^{*} \mathbf{b}}^{\lambda}=\left(T_{\mathbf{b}}^{\lambda}\right)^{*} T_{\mathbf{b}}^{\lambda}+R^{\lambda}$, where $R^{\lambda}$ is of order $\leq 1$. Consequently, we have the estimate of the form

$$
\|\psi\|_{1, \lambda} \leq C\left(K_{0}\right)\left\|T_{\mathbf{b}}^{\lambda} \psi\right\|_{0}
$$

For all $\lambda \geq \lambda_{0}$, we thus obtain

$$
\begin{aligned}
\|\psi\|_{1, \lambda} & \leq C\left(K_{0}\right)\left(\left\|T_{\mathbf{b}}^{\lambda} \psi+\left.T_{\mathbf{M}}^{\lambda} W\right|_{x_{2}=0}\right\|_{0}+\left\|\left.W^{n c}\right|_{x_{2}=0}\right\|\right) \\
& \leq C\left(K_{0}\right)\left(\left.\frac{1}{\lambda}\left\|T_{\mathbf{b}}^{\lambda} \psi+\left.T_{\mathbf{M}}^{\lambda} W\right|_{x_{2}=0}\right\|\left\|_{1, \lambda}+\right\| W^{n c}\right|_{x_{2}=0} \|\right) .
\end{aligned}
$$

From (6.18) and (6.21), we deduce for $\lambda \geq \lambda_{0}$ large enough (depending on $K_{0}$ ) the estimate:

$$
\|\psi\|_{1, \lambda} \leq C\left(K_{0}\right)\left(\frac{1}{\lambda}\left\|\mathcal{B}^{\lambda}(W, \psi)\right\|_{1, \lambda}+\left\|\left.W^{n c}\right|_{x_{2}=0}\right\|\right)
$$

which shows that it only remains to prove the estimate of $\left.W^{n c}\right|_{x_{2}=0}$ in terms of the source terms, which will be done by the paralinearized system $(6.25)$.

For all $(\tau, \eta)$ in the hemisphere $\Sigma$, we define the matrix

$$
\Pi\left(t, x_{1}, \delta, \eta, \lambda\right):=\left(\begin{array}{lcc}
0 & 0 & 1 \\
\tau+i \eta v_{r}\left(t, x_{1}, 0\right) & -i \eta\left(v_{r}-v_{l}\right)\left(t, x_{1}, 0\right) & 0
\end{array}\right),
$$

and we extend $\Pi$ as a homogeneous mapping of degree 0 with respect to $(\tau, \eta)$. We have $\Pi \mathbf{b} \equiv 0$ (here $\Pi \mathbf{b}$ denotes matrix $\Pi$ multiplied by $\mathbf{b}$ ), and $\Pi \in \Gamma_{2}^{0}$ (here $\Pi$ plays the same 
role of $Q(\tau, \eta)$ defined in (4.8) for the constant coefficients problem). Here, $\Gamma_{k}^{m}$ denotes the set of paradifferential symbols $a(x, \xi, \gamma): \mathbb{R}^{2} \times \mathbb{R}^{2} \times[0, \infty) \rightarrow \mathbb{C}^{N \times N}$ of degree $m \in \mathbb{R}$ and regularity $k \in \mathbb{N}$ such that $a$ is $C^{\infty}$ with respect to $\xi$ and for all $\alpha \in \mathbb{N}^{2}$ and there exists a constant $C_{\alpha}$ satisfying

$$
\left\|\partial_{\xi}^{\alpha} a(\cdot, \xi, \gamma)\right\|_{W^{k, \infty}\left(\mathbb{R}^{2}\right)} \leq C_{\alpha}\left(\gamma^{2}+|\xi|^{2}\right)^{-\frac{m-|\alpha|}{2}} .
$$

We thus obtain

$$
\begin{aligned}
& \left\|T_{\Pi}^{\lambda} T_{\mathbf{b}}^{\lambda} \psi\right\|_{1, \lambda}=\left\|T_{\Pi}^{\lambda} T_{\mathbf{b}}^{\lambda} \psi-T_{\Pi \mathbf{b}}^{\lambda} \psi\right\|_{1, \lambda} \leq C\left(K_{0}\right)\|\psi\|_{1, \lambda}, \\
& \left\|\left.T_{\Pi \mathbf{M}}^{\lambda} W\right|_{x_{2}=0}-\left.T_{\Pi}^{\lambda} T_{\mathbf{M}}^{\lambda} W\right|_{x_{2}=0}\right\|_{1, \lambda} \leq C\left(K_{0}\right)\left\|\left.W^{n c}\right|_{x_{2}=0}\right\|_{0} .
\end{aligned}
$$

Using the decomposition

$$
\left.T_{\Pi \mathbf{M}}^{\lambda} W\right|_{x_{2}=0}=\left.\left(T_{\Pi \mathbf{M}}^{\lambda}-T_{\Pi}^{\lambda} T_{\mathbf{M}}^{\lambda}\right) W\right|_{x_{2}=0}+T_{\Pi}^{\lambda}\left(\left.T_{\mathbf{M}}^{\lambda} W\right|_{x_{2}=0}+T_{\mathbf{b}}^{\lambda} \psi\right)-T_{\Pi}^{\lambda} T_{\mathbf{b}}^{\lambda} \psi,
$$

we get the following estimate:

$$
\begin{aligned}
& \left\|\left.T_{\Pi \mathbf{M}}^{\lambda} W\right|_{x_{2}=0}\right\|_{1, \lambda} \\
\leq & C\left(K_{0}\right)\left(\left\|\left.W^{n c}\right|_{x_{2}=0}\right\|_{0}+\left\|T_{\mathbf{b}}^{\lambda} \psi+\left.T_{\mathbf{M}}^{\lambda} W\right|_{x_{2}=0}\right\|_{1, \lambda}+\|\psi\|_{1, \lambda}\right) .
\end{aligned}
$$

As was done in the case of constant coefficients, we define the symbol $\beta$ of the reduced boundary conditions for $\left(t, x_{1}, \delta, \eta, \lambda\right) \in \mathbb{R}^{4} \times \mathbb{R}^{+}$:

$$
\beta\left(t, x_{1}, \delta, \eta, \lambda\right):=\left(\beta^{r}, \beta^{l}\right)\left(t, x_{1}, \delta, \eta, \lambda\right),
$$

where

$$
\beta^{r}\left(t, x_{1}, \delta, \eta, \lambda\right):=\left(\begin{array}{cccr}
0 & 0 & \left(1+\frac{m_{r}}{n_{r}}\right)\left\langle\partial_{1} \varphi\right\rangle & \left(1+\frac{m_{r}}{n_{r}}\right)\left\langle\partial_{1} \varphi\right\rangle \\
0 & 0 & -\left(\tau+i \eta v_{l}\right) \frac{c_{r}}{n_{r}}\left\langle\partial_{1} \varphi\right\rangle^{2} & \left(\tau+i \eta v_{l}\right) \frac{c_{r}}{n_{r}}\left\langle\partial_{1} \varphi\right\rangle^{2}
\end{array}\right)
$$

and

$$
\beta^{l}\left(t, x_{1}, \delta, \eta, \lambda\right):=\left(\begin{array}{cccr}
0 & 0 & -\left(1+\frac{m_{l}}{n_{l}}\right)\left\langle\partial_{1} \varphi\right\rangle & -\left(1+\frac{m_{l}}{n_{l}}\right)\left\langle\partial_{1} \varphi\right\rangle \\
0 & 0 & \left(\tau+i \eta v_{r}\right) \frac{c_{l}}{n_{l}}\left\langle\partial_{1} \varphi\right\rangle^{2} & -\left(\tau+i \eta v_{r}\right) \frac{c_{l}}{n_{l}}\left\langle\partial_{1} \varphi\right\rangle^{2}
\end{array}\right) .
$$

Notice that $v_{r}\left(t, x_{1}, 0\right) \neq-v_{l}\left(t, x_{1}, 0\right)$ here.

We now focus on the paralinearized system with reduced boundary conditions:

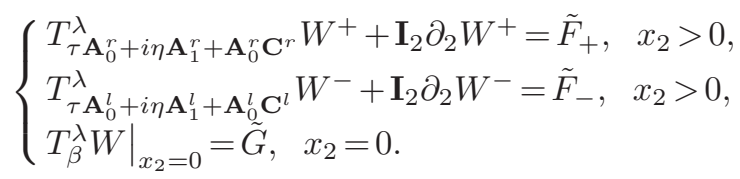

Notice that the new boundary condition $(6.25)_{3}$ does not involve the front $\psi$ since the map $\Pi\left(t, x_{1}, \delta, \eta, \lambda\right)$ is introduced. In addition, $T_{\tau \mathbf{A}_{0}^{r}+i \eta \mathbf{A}_{1}^{r}+\mathbf{A}_{0}^{r} \mathbf{C}^{r}}^{\lambda}=T_{\tau \mathbf{A}_{0}^{r}+i \eta \mathbf{A}_{1}^{r}}^{\lambda}+T_{\mathbf{A}_{0}^{r} \mathbf{C}^{r}}^{\lambda}$.

6.3. Estimate for the paralinearized problem and microlocalization. In order to prove Theorem 5.1, we first need to establish an estimate for the paralinearized problem (6.25). More precisely, we have the following proposition. 
Proposition 6.2. There exists a constant $C_{0}$, depending only on $K_{0}$ and $\kappa_{0}$, such that the solution $W$ to (6.25) satisfies

$$
\left\|\left.W^{n c}\right|_{x_{2}=0}\right\|_{0}^{2} \leq C_{0}\left(\frac{1}{\lambda^{3}}\left\|\left|\tilde{F}\left\|_{1, \lambda}^{2}+\frac{1}{\lambda^{2}}\right\|\right| \tilde{G}\right\|_{1, \lambda}^{2}\right)
$$

for all $\lambda \geq \lambda_{0}$ (where $\lambda_{0}$ only depends on $K_{0}$ and $\left.\kappa_{0}\right)$.

Recall that the boundary matrix $\beta$ in (6.25) only acts on $W^{n c}=\left(W_{3}^{+}, W_{4}^{+}, W_{3}^{-}, W_{4}^{-}\right)$and not on the full vector $W$. Namely, the first and fourth columns of $\beta$ vanish (see (6.24)). Consequently, we can write the boundary conditions as the form:

$$
\left.T_{\beta^{n c}}^{\lambda} W^{n c}\right|_{x_{2}=0}=\tilde{G},
$$

that is, we consider $\beta^{n c}$ as a matrix with only four columns and two rows:

$$
\beta^{n c}\left(t, x_{1}, \delta, \eta, \lambda\right):=\left(\beta^{n c, r}, \beta^{n c, l}\right)\left(t, x_{1}, \delta, \eta, \lambda\right),
$$

where

and

$$
\beta^{n c, r}\left(t, x_{1}, \delta, \eta, \lambda\right):=\left(\begin{array}{lr}
\left(1+\frac{m_{r}}{n_{r}}\right)\left\langle\partial_{1} \varphi\right\rangle & \left(1+\frac{m_{r}}{n_{r}}\right)\left\langle\partial_{1} \varphi\right\rangle \\
-\left(\tau+i \eta v_{l}\right) \frac{c_{r}}{n_{r}}\left\langle\partial_{1} \varphi\right\rangle^{2} & \left(\tau+i \eta v_{l}\right) \frac{c_{r}}{n_{r}}\left\langle\partial_{1} \varphi\right\rangle^{2}
\end{array}\right)
$$

$$
\beta^{n c, l}\left(t, x_{1}, \delta, \eta, \lambda\right):=\left(\begin{array}{cr}
-\left(1+\frac{m_{l}}{n_{l}}\right)\left\langle\partial_{1} \varphi\right\rangle & -\left(1+\frac{m_{l}}{n_{l}}\right)\left\langle\partial_{1} \varphi\right\rangle \\
\left(\tau+i \eta v_{r}\right) \frac{c_{l}}{n_{l}}\left\langle\partial_{1} \varphi\right\rangle^{2} & -\left(\tau+i \eta v_{r}\right) \frac{c_{l}}{n_{l}}\left\langle\partial_{1} \varphi\right\rangle^{2}
\end{array}\right) .
$$

We now prove Proposition 6.2, that is, estimate (6.26) for the paralinearized system (6.25) using microlocalization. The proof is similar to Subsection 4.2.

6.3.1. Reduction to an ODE system. To derive the desired energy estimate for (6.25), we follow the general strategy of the constant coefficient case. First, it is easy to get from the definition of $\mathbf{A}_{0}^{r}$ and $\mathbf{A}_{1}^{r}$ in (6.5)

$$
\tau \mathbf{A}_{0}^{r}+i \eta \mathbf{A}_{1}^{r}=\left(\begin{array}{cccr}
\tau+i v_{r} \eta & 0 & 0 & 0 \\
0 & \tau+i v_{r} \eta & i \eta \frac{c_{r}^{2}}{n_{r}\left\langle\partial_{1} \Phi_{r}\right\rangle} & i \eta \frac{c_{r}^{2}}{n_{r}\left\langle\partial_{1} \Phi_{r}\right\rangle} \\
0 & i \eta \frac{n_{r}}{2 c_{r}} \frac{\partial_{2} \Phi_{r}}{\left\langle\partial_{1} \Phi_{r}\right\rangle^{2}} & \left(\tau \mathbf{A}_{0}^{r}+i \eta \mathbf{A}_{1}^{r}\right)_{33} & 0 \\
0 & -i \eta \frac{n_{r}}{2 c_{r}} \frac{\partial_{2} \Phi_{r}}{\left\langle\partial_{1} \Phi_{r}\right\rangle^{2}} & 0 & \left(\tau \mathbf{A}_{0}^{r}+i \eta \mathbf{A}_{1}^{r}\right)_{44}
\end{array}\right)
$$

where

$$
\begin{aligned}
\left(\tau \mathbf{A}_{0}^{r}+i \eta \mathbf{A}_{1}^{r}\right)_{33} & =-\left(\tau \mathbf{A}_{0}^{r}+i \eta \mathbf{A}_{1}^{r}\right)_{44} \\
& =\frac{\left(\tau+i v_{r} \eta\right) \partial_{2} \Phi_{r}}{c_{r}\left\langle\partial_{1} \Phi_{r}\right\rangle}-\frac{\partial_{2} \Phi_{r} \partial_{1} \Phi_{r} i \eta}{\left\langle\partial_{1} \Phi_{r}\right\rangle^{2}}
\end{aligned}
$$

Thus, we can obtain the two equations in (6.25) which do not involve any $x_{2}$ derivative since $I_{2}=\operatorname{diag}(0,0,1,1)$ :

$$
\begin{aligned}
& T_{\tau+i v_{r} \eta}^{\lambda} W_{2}^{+}+T_{i \eta \frac{c_{r}^{2}}{n_{r}\left\langle\partial_{1} \Phi_{r}\right\rangle}}^{\lambda} W_{3}^{+}+T_{i \eta_{\frac{c_{r}}{n_{r}\left\langle\partial_{1} \Phi_{r}\right\rangle}}^{\lambda}} W_{4}^{+}+\text {order } 0 \text { terms }=F_{2}^{+} \text {, } \\
& T_{\tau+i v_{l} \eta}^{\lambda} W_{2}^{-}+T_{i \eta \frac{c_{l}^{2}}{n_{l}\left\langle\partial_{1} \Phi_{l}\right\rangle}}^{\lambda} W_{i \eta_{\frac{1}{n_{l}\left\langle\partial_{1} \Phi_{l}\right\rangle}}^{-}+T^{\lambda}}^{\lambda} W_{4}^{-}+\text {order } 0 \text { terms }=F_{2}^{-} \text {. }
\end{aligned}
$$


By formally inverting the operators $T_{\tau+i v_{r, l} \eta}^{\lambda}$ and substituting the corresponding value of $W_{2}^{ \pm}$into the four remaining equations (the third, fourth, seventh, and eighth ones), one obtains a system of the form

$$
\left\{\begin{array}{l}
\partial_{2} W^{n c}=T_{\mathbb{A}}^{\lambda} W^{n c}+T_{\mathbb{E}}^{\lambda} W^{n c}+\text { source term, } \quad x_{2}>0, \\
\left.T_{\beta^{n c}}^{\lambda} W^{n c}\right|_{x_{2}=0}=\text { source term, } \quad x_{2}=0,
\end{array}\right.
$$

where $\mathbb{A}$ is of degree 1 and $\mathbb{E}$ is of degree zero. Recall that $\beta^{n c}$ is only a $2 \times 4$ matrix since the boundary matrix $\beta$ in (6.25) only acts on $W^{n c}$ and $T_{\mathbb{A}}^{\lambda} W^{n c}$ and $T_{\mathbb{E}}^{\lambda} W^{n c}$ are "the first-order term" and "the zeroth-order term," respectively. The matrices $\mathbb{A}$ and $\mathbb{E}$ are block diagonal since the equations for $W^{+}$and $W^{-}$are decoupled. When inverting the operators $T_{\tau+i v_{r, l} \eta}^{\lambda}$, we need to consider the zeroth order terms in order to avoid introducing $W_{2}^{ \pm}$in the final equation for $W^{n c}$. Let us now consider the first-order term and find explicitly the symbol $\mathbb{A}$. Take the following $2 \times 2$ matrix:

$$
\mathbb{A}^{r}:=\left(\begin{array}{cc}
\mathbb{A}_{1}^{r} & -\mathbb{A}_{3}^{r} \\
\mathbb{A}_{3}^{r} & \mathbb{A}_{2}^{r}
\end{array}\right)
$$

with

$$
\begin{aligned}
\mathbb{A}_{1}^{r} & :=-\frac{c_{r} \eta^{2} \partial_{2} \Phi_{r}}{2\left(\tau+i v_{r} \eta\right)\left\langle\partial_{1} \Phi_{r}\right\rangle^{3}}-\frac{\left(\tau+i v_{r} \eta\right) \partial_{2} \Phi_{r}}{c_{r}\left\langle\partial_{1} \Phi_{r}\right\rangle}+\frac{\partial_{2} \Phi_{r} \partial_{1} \Phi_{r} i \eta}{\left\langle\partial_{1} \Phi_{r}\right\rangle^{2}} \\
\mathbb{A}_{2}^{r} & :=\frac{c_{r} \eta^{2} \partial_{2} \Phi_{r}}{2\left(\tau+i v_{r} \eta\right)\left\langle\partial_{1} \Phi_{r}\right\rangle^{3}}+\frac{\left(\tau+i v_{r} \eta\right) \partial_{2} \Phi_{r}}{c_{r}\left\langle\partial_{1} \Phi_{r}\right\rangle}+\frac{\partial_{2} \Phi_{r} \partial_{1} \Phi_{r} i \eta}{\left\langle\partial_{1} \Phi_{r}\right\rangle^{2}}, \\
\mathbb{A}_{3}^{r} & :=\frac{c_{r} \eta^{2} \partial_{2} \Phi_{r}}{2\left(\tau+i v_{r} \eta\right)\left\langle\partial_{1} \Phi_{r}\right\rangle^{3}} .
\end{aligned}
$$

The definition of $\mathbb{A}^{l}$ is completely similar by changing the index $r$ by $l$. The symbol $\mathbb{A}$ mentioned above is just the block diagonal matrix

$$
\mathbb{A}:=\left(\begin{array}{ll}
\mathbb{A}^{r} & 0 \\
0 & \mathbb{A}^{l}
\end{array}\right)
$$

6.3.2. Microlocal analysis in the neighborhood of the pole and bicharacteristic curve. The set of poles of $\mathbb{A}$ is denoted by $\mathcal{Y}_{p}$, that is,

$$
\mathcal{Y}_{p}:=\left\{\left(t, x_{1}, x_{2}, \tau, \eta\right) \in \bar{\Omega} \times \Xi \text { such that } \tau=-i \eta v_{r, l}\left(t, x_{1}, x_{2}\right)\right\} .
$$

As in the constant coefficient case, we denote by $E^{-}\left(t, x_{1}, x_{2}, \tau, \eta\right)$ the stable subspace of $\mathbb{A}\left(t, x_{1}, x_{2}, \tau, \eta\right)$. This stable subspace is well defined when $\mathfrak{R} \tau>0$, and admits a continuous extension up to any $(\tau, \eta)$ such that $\tau \in i \mathbb{R}$ and $(\tau, \eta) \neq(0,0)$.

The stable eigenvalues $\omega_{r, l}^{-}$of $\mathbb{A}\left(t, x_{1}, x_{2}, \tau, \eta\right)$ fulfill the dispersion relations

$$
\left(\omega_{r, l}^{-}\right)^{2}-\left(\mathbb{A}_{1}^{r, l}+\mathbb{A}_{2}^{r, l}\right) \omega_{r, l}^{-}+\mathbb{A}_{1}^{r, l} \mathbb{A}_{2}^{r, l}+\left(\mathbb{A}_{3}^{r, l}\right)^{2}=0
$$

That is,

$$
\left(\omega_{r, l}^{-}\right)^{2}-2 i \eta \frac{\partial_{1} \Phi_{r, l} \partial_{2} \Phi_{r, l}}{\left\langle\partial_{1} \Phi_{r, l}\right\rangle^{2}} \omega_{r, l}^{-}-\frac{\left(\partial_{2} \Phi_{r, l}\right)^{2}}{\left\langle\partial_{1} \Phi_{r, l}\right\rangle^{2}}\left(\eta^{2}+\frac{1}{c_{r, l}^{2}}\left(\tau+i v_{r, l} \eta\right)^{2}\right)=0 .
$$

Introduce

$$
\widetilde{\omega}_{r, l}^{-}=\frac{\left\langle\partial_{1} \Phi_{r, l}\right\rangle^{2}}{\partial_{2} \Phi_{r, l}}\left(\omega_{r, l}^{-}-i \eta \frac{\partial_{1} \Phi_{r, l} \partial_{2} \Phi_{r, l}}{\left\langle\partial_{1} \Phi_{r, l}\right\rangle^{2}}\right), \quad \tilde{c}_{r, l}=\frac{c_{r, l}}{\left\langle\partial_{1} \Phi_{r, l}\right\rangle} .
$$


Then, we get

$$
\left(\widetilde{\omega}_{r, l}^{-}\right)^{2}=\frac{1}{\tilde{c}_{r, l}^{2}}\left(\tau+i v_{r, l} \eta\right)^{2}+\eta^{2}
$$

and

$$
\omega_{r, l}^{-} I_{2 \times 2}-\mathbb{A}^{r, l}=\frac{\partial_{2} \Phi_{r, l}}{\left\langle\partial_{1} \Phi_{r, l}\right\rangle^{2}}\left(\begin{array}{cc}
\widetilde{\omega}_{r, l}^{-}+\frac{\tilde{c}_{r, l} \eta^{2}}{2\left(\tau+i v_{r, l} \eta\right)}+\frac{\tau+i v_{r, l} \eta}{\tilde{c}_{r, l}} & \frac{\tilde{c}_{r, l} \eta^{2}}{2\left(\tau+i v_{r, l} \eta\right)} \\
-\frac{\tilde{c}_{r, l} \eta^{2}}{2\left(\tau+i v_{r, l} \eta\right)} & \widetilde{\omega}_{r, l}^{-}-\frac{\tilde{c}_{r, l} \eta^{2}}{2\left(\tau+i v_{r, l} \eta\right)}-\frac{\tau+i v_{r, l} \eta}{\tilde{c}_{r, l}}
\end{array}\right) .
$$

The corresponding stable eigenspace are spanned by

$$
\begin{aligned}
& E_{r}(\tau, \eta)=\left(\left(\tau+i v_{r} \eta\right) \widetilde{\omega}_{r}^{-}-\left\{\frac{1}{\tilde{c}_{r}}\left(\tau+i v_{r} \eta\right)^{2}+\frac{\tilde{c}_{r}}{2} \eta^{2}\right\}, \frac{\tilde{c}_{r}}{2} \eta^{2}, 0,0\right)^{T}, \\
& E_{l}(\tau, \eta)=\left(0,0, \frac{\tilde{c}_{l}}{2} \eta^{2},\left(\tau+i v_{l} \eta\right) \widetilde{\omega}_{l}^{-}-\left\{\frac{1}{\tilde{c}_{l}}\left(\tau+i v_{l} \eta\right)^{2}+\frac{\tilde{c}_{l}}{2} \eta^{2}\right\}\right)^{T} .
\end{aligned}
$$

The Lopatinskii determinant of problem (6.30) is defined by

$$
\begin{aligned}
& \operatorname{det}\left(\beta^{n c}\left(E_{r} \quad E_{l}\right)\right) \\
= & \left\langle\partial_{1} \varphi\right\rangle^{4} \operatorname{det}\left(\begin{array}{ll}
L_{11} & L_{12} \\
L_{21} & L_{22}
\end{array}\right) \\
= & \left\langle\partial_{1} \varphi\right\rangle^{4} \frac{m_{r}+n_{r}}{n_{r} n_{l}} \tilde{c}_{l} \tilde{c}_{r}\left(\widetilde{\omega}_{r}^{-}+\widetilde{\omega}_{l}^{-}\right)\left(\widetilde{\omega}_{l}^{-} \widetilde{\omega}_{r}^{-}-\eta^{2}\right)\left(\tilde{c}_{r} \widetilde{\omega}_{r}^{-}-\tau-i v_{r} \eta\right)\left(\tilde{c}_{l} \widetilde{\omega}_{l}^{-}-\tau-i v_{l} \eta\right) .
\end{aligned}
$$

where

$$
\begin{aligned}
& L_{11}=\left(1+\frac{m_{r}}{n_{r}}\right)\left(\left(\tau+i v_{r} \eta\right) \widetilde{\omega}_{r}-\frac{1}{\tilde{c}_{r}}\left(\tau+i v_{r} \eta\right)^{2}\right), \\
& L_{12}=-\left(1+\frac{m_{l}}{n_{l}}\right)\left(\left(\tau+i v_{l} \eta\right) \widetilde{\omega}_{l}-\frac{1}{\tilde{c}_{l}}\left(\tau+i v_{l} \eta\right)^{2}\right), \\
& L_{21}=\frac{\tilde{c}_{r}}{n_{r}}\left(\tau+i v_{l} \eta\right)\left(-\left(\tau+i v_{r} \eta\right) \widetilde{\omega}_{r}+\frac{1}{\tilde{c}_{r}}\left(\tau+i v_{r} \eta\right)^{2}+\tilde{c}_{r} \eta^{2}\right), \\
& L_{22}=-\frac{\tilde{c}_{l}}{n_{l}}\left(\tau+i v_{r} \eta\right)\left(\left(\tau+i v_{l} \eta\right) \widetilde{\omega}_{l}-\left(\frac{1}{\tilde{c}_{l}}\left(\tau+i v_{l} \eta\right)^{2}+\tilde{c}_{l} \eta^{2}\right)\right) .
\end{aligned}
$$

We used the fact $m_{r}+n_{r}=m_{l}+n_{l}$ in the last equality.

Formally, the Lopatinskii determinant has the same zero points as those in [44]. Thus, we can directly use their corresponding results, which are summarized as follows.

Proposition 6.3 (see [44], Proposition 4.4). Assume that

$$
\bar{v}_{r}-\bar{v}_{l}>\left(\bar{c}_{r}^{2 / 3}+\bar{c}_{l}^{2 / 3}\right)^{3 / 2}
$$

and the perturbations $\dot{U}_{r, l}, \nabla \Phi_{r, l}$ have compact support and satisfy (5.2) with sufficiently small $K_{0}$. Then, there exist two functions $X_{2}, X_{3} \in W^{2, \infty}\left(\mathbb{R}^{2}\right)$ such that, for every $\left(t, x_{1}\right) \in \mathbb{R}^{2}$, the following inequalities hold true at $\left(t, x_{1}, 0\right)$ :

$$
\frac{c_{r}}{\left\langle\partial_{1} \varphi\right\rangle}-\frac{v_{r}-v_{l}}{2}<\frac{c_{r} X_{2}}{\left\langle\partial_{1} \varphi\right\rangle}<\frac{c_{r} X_{3}}{\left\langle\partial_{1} \varphi\right\rangle}<-\frac{c_{l}}{\left\langle\partial_{1} \varphi\right\rangle}+\frac{v_{r}-v_{l}}{2}
$$


For any fixed $\left(t, x_{1}\right) \in \mathbb{R}^{2}, \Delta\left(t, x_{1}, \tau, \eta\right)$ vanishes at a point $(\tau, \eta) \in \Sigma$ if and only if one of the following three identities is satisfied:

$$
\begin{array}{ll}
\text { (i) } \tau=\eta\left[q\left(t, x_{1}\right) \frac{\left(v_{r}-v_{l}\right)\left(t, x_{1}, 0\right)}{2}-\frac{\left(v_{r}+v_{l}\right)\left(t, x_{1}, 0\right)}{2}\right], \quad \eta \neq 0, & \\
\text { (ii) } \tau=\eta\left[\frac{c_{r}\left(t, x_{1}, 0\right) X_{2}\left(t, x_{1}\right)}{\left\langle\partial_{1} \varphi\left(t, x_{1}\right)\right\rangle}-\frac{\left(v_{r}+v_{l}\right)\left(t, x_{1}, 0\right)}{2}\right], & \eta \neq 0, \\
\text { (iii) } \tau=\eta\left[\frac{c_{r}\left(t, x_{1}, 0\right) X_{3}\left(t, x_{1}\right)}{\left\langle\partial_{1} \varphi\left(t, x_{1}\right)\right\rangle}-\frac{\left(v_{r}+v_{l}\right)\left(t, x_{1}, 0\right)}{2}\right], & \eta \neq 0,
\end{array}
$$

where $q\left(t, x_{1}\right)=\frac{\left(c_{r}-c_{l}\right)\left(t, x_{1}, 0\right)}{\left(c_{r}+c_{l}\right)\left(t, x_{1}, 0\right)}$. For every $\left(t, x_{1}\right) \in \mathbb{R}^{2}$, the three sets of boundary frequencies $(\tau, \eta)$ defined by (i)-(iii) are mutually disjoint, as long as

$$
\frac{\left(v_{r}-v_{l}\right)\left(t, x_{1}, 0\right)}{2} \neq \frac{\left(c_{r}+c_{l}\right)\left(t, x_{1}, 0\right)}{2} .
$$

Thus, $\Delta\left(t, x_{1}, \cdot, \cdot\right)$ has only simple roots.

If, on the contrary, the point $\left(t, x_{1}\right) \in \mathbb{R}^{2}$ satisfies

$$
\frac{\left(v_{r}-v_{l}\right)\left(t, x_{1}, 0\right)}{2}=\frac{\left(c_{r}+c_{l}\right)\left(t, x_{1}, 0\right)}{2},
$$

then one of the following two identities hold at $\left(t, x_{1}, 0\right)$.

$$
q \frac{v_{r}-v_{l}}{2}=\frac{c_{r} X_{2}}{\left\langle\partial_{1} \varphi\right\rangle}, \quad \text { or } \quad q \frac{v_{r}-v_{l}}{2}=\frac{c_{l} X_{3}}{\left\langle\partial_{1} \varphi\right\rangle} .
$$

This means that, for $\left(t, x_{1}\right)$ satisfying (6.42), any point $\left(\tau_{0}, \eta_{0}\right)$ defined by condition (i) is a quadratic root of $\Delta\left(t, x_{1}, \tau, \eta\right)=0$; namely there exist a neighborhood $\mathcal{V}_{0} \subset \Sigma$ of $\left(\tau_{0}, \eta_{0}\right)$ and a $C^{\infty}$ function $h\left(t, x_{1}, \cdot, \cdot\right)$ such that

$$
\Delta\left(t, x_{1}, \tau, \eta\right)=\left(\tau-\tau_{0}\right)^{2} h\left(t, x_{1}, \tau, \eta\right), \quad \forall(\tau, \eta) \in \mathcal{V}_{0}
$$

and $h\left(t, x_{1}, \tau_{0}, \eta_{0}\right) \neq 0$.

We define the critical set of space frequency variables as follows:

$$
\mathcal{Y}_{c}^{0}:=\left\{\left(t, x_{1}, \tau, \eta\right) \in \partial \Omega \times \Xi\right\}
$$

such that

$$
\tau \in\left\{i \eta\left(q \frac{v_{r}-v_{l}}{2}-\frac{v_{r}+v_{l}}{2}\right)\left(t, x_{1}, 0\right), \quad i \eta\left(\frac{c_{r} X_{2,3}}{\left\langle\partial_{1} \varphi\right\rangle}-\frac{v_{r}+v_{l}}{2}\right)\left(t, x_{1}, 0\right)\right\} .
$$

If the perturbation $\left(\dot{U}_{r, l}, \nabla \Phi_{r, l}\right)$ is sufficiently small in the $L^{\infty}$ norm, one has

$$
\mathcal{Y}_{c}^{0} \cap\left(\mathcal{Y}_{p} \cap\left\{x_{2}=0\right\}\right)=\emptyset .
$$

There also exists a neighborhood $\mathcal{V}_{c}^{0}$ of $\mathcal{Y}_{c}^{0}$ in $\mathbb{R}^{2} \times \Xi$ and a mapping $Q_{0}$ on $\mathcal{V}_{c}^{0}$ with values in the set of $4 \times 4$ invertible matrices and homogeneous of degree 0 with respect to $(\tau, \eta)$ such that

$$
Q_{0}(z) \mathbb{A}(z) Q_{0}(z)^{-1}=\operatorname{diag}\left(\omega_{r}^{-}(z), \omega_{r}^{+}(z), \omega_{l}^{-}(z), \omega_{l}^{+}(z)\right)
$$

for any $z=\left(t, x_{1}, \tau, \eta\right) \in \mathcal{V}_{c}^{0}$, where $\omega_{r}^{-}$is the eigenvalue with negative real part of $\mathbb{A}^{r}$ when $\lambda>0$. 
The propagation of singularity on the boundary $\partial \Omega$ is formally the same as the isentropic Euler case [11, Proposition 5.4]. Hence, the singularities propagate along the solutions of the Hamiltonian system associated with $h=\Im \omega_{r, l}^{-}$.

Proposition 6.4 (see [44]). Assume that the perturbation $\left(\dot{U}_{r, l}, \nabla \Phi_{r, l}\right)$ is small in $W^{2, \infty}(\Omega)$ and has compact support. Then, one can choose the neighborhood $\mathcal{V}_{c}^{0}$ such that there exists an open set $\mathcal{V}_{c} \subset \bar{\Omega} \times \Xi$ satisfying the following properties:

- $\mathcal{V}_{c} \cap\left\{x_{2}=0\right\}=\mathcal{V}_{c}^{0}$ and $\mathcal{V}_{c} \cap \mathcal{Y}_{p}=\emptyset$.

- The symbol $\mathbb{A}$ defined above is diagonalizable on the set $\mathcal{V}_{c}$. That is, (6.45) holds on all $\mathcal{V}_{c}$, and not only on the trace $\mathcal{V}_{c}^{0}$.

- For all $z=\left(t, x_{1}, x_{2}, \tau, \eta\right) \in \mathcal{V}_{c}$, one has

$$
\omega_{r}^{-}(z) \neq \omega_{r}^{+}(z) \quad \text { and } \quad \omega_{l}^{-}(z) \neq \omega_{l}^{+}(z) .
$$

- The solutions of the Hamiltonian system of ODEs

$$
\begin{aligned}
\frac{d t}{d x_{2}} & =\frac{\partial h}{\partial \delta}\left(t, x_{1}, x_{2}, \tau, \eta\right) \\
\frac{d x_{1}}{d x_{2}} & =\frac{\partial h}{\partial \eta}\left(t, x_{1}, x_{2}, \tau, \eta\right) \\
\frac{d \delta}{d x_{2}} & =-\frac{\partial h}{\partial t}\left(t, x_{1}, x_{2}, \tau, \eta\right), \\
\frac{d \eta}{d x_{2}} & =-\frac{\partial h}{\partial x_{1}}\left(t, x_{1}, x_{2}, \tau, \eta\right),\left.\quad\left(t, x_{1}, \delta, \eta, \lambda\right)\right|_{x_{2}=0} \in \mathcal{V}_{c}^{0}
\end{aligned}
$$

are defined for all $x_{2} \geq 0$ and remain in $\mathcal{V}_{c}$ both for $h=\Im \omega_{r}^{-}$and $h=\Im \omega_{l}^{-}$. These solutions are referred to as bicharacteristic curves.

From Proposition 6.3, we know that $\mathcal{Y}_{c}^{0}$ and $\left\{\frac{v_{r}-v_{l}}{2}=\frac{c_{r}+c_{l}}{2}\right\}$ are disjoint, and we will construct a weight that vanishes on the bicharacteristic curves and satisfies a transport equation. Define $\sigma$ on $\mathbb{R}^{2} \times \Sigma$ by setting

$$
\begin{aligned}
\sigma(z):= & {\left[\delta-\eta\left(q \frac{v_{r}-v_{l}}{2}-\frac{v_{r}+v_{l}}{2}\right)\left(t, x_{1}, 0\right)\right] \cdot\left[\delta-\eta\left(\tilde{c}_{r} X_{2}-\frac{v_{r}+v_{l}}{2}\right)\left(t, x_{1}, 0\right)\right] } \\
& \cdot\left[\delta-\eta\left(\tilde{c}_{r} X_{3}-\frac{v_{r}+v_{l}}{2}\right)\left(t, x_{1}, 0\right)\right]
\end{aligned}
$$

for $z=\left(t, x_{1}, \tau, \eta\right) \in \mathbb{R}^{2} \times \Sigma$. Then, we extend $\sigma(z)$ to the whole set $\mathbb{R}^{2} \times \Xi$ as a homogeneous mapping of degree one with respect to $(\tau, \eta)$. Hence, $\sigma(z) \in \Gamma_{2}^{1}$. Let $\sigma_{r, l}$ be the solutions to the linear transport equations

$$
\begin{aligned}
& \partial_{2} \sigma_{r}+\left\{\sigma_{r},-\Im \omega_{r}^{-}\right\}=0, \quad \partial_{2} \sigma_{l}+\left\{\sigma_{l},-\Im \omega_{l}^{-}\right\}=0, \\
& \left.\sigma_{r}\right|_{x_{2}=0}=\left.\sigma_{l}\right|_{x_{2}=0}=\sigma,
\end{aligned}
$$

where

$$
\{a, b\}=-\frac{\partial a}{\partial \delta} \frac{\partial b}{\partial t}-\frac{\partial a}{\partial \eta} \frac{\partial b}{\partial x_{1}}+\frac{\partial a}{\partial t} \frac{\partial b}{\partial \delta}+\frac{\partial a}{\partial x_{1}} \frac{\partial b}{\partial \eta}
$$

is the Poisson bracket of $a$ and $b$. Shrinking $\mathcal{V}_{c}^{0}$ and $\mathcal{V}_{c}$ if necessary, we may assume that $\sigma_{r}$ and $\sigma_{l}$ are defined in the whole open set $\mathcal{V}_{c}$. Note that $\sigma_{r}$ vanishes on the 
bicharacteristic curve originating from $\mathcal{Y}_{c}^{0}$ and associated with the symbol $\Im \omega_{r}^{-}$. Far from these bicharacteristic curves, both $\left|\sigma_{r}\right|$ and $\left|\sigma_{l}\right|$ are bounded from below. To extend $\sigma_{r, l}$ and $\mathbb{Q}_{0}$ to the whole frequency space, we introduce the cut-off functions $\chi_{c}$ and $\chi_{p}$ satisfying the following properties:

- $\chi_{c}$ and $\chi_{p}$ are smooth, that is, $C^{\infty}$ and homogeneous of degree 0 with respect to $(\tau, \eta)$. Thus, they belong to the class $\Gamma_{k}^{0}$ for any integer $k$.

- The support of $\chi_{c}$ is contained in the open set $\mathcal{V}_{c}$ and $\chi_{c} \equiv 1$ in a neighborhood of the bicharacteristic curves originating from $\mathcal{Y}_{c}^{0}$.

- The support of $\chi_{p}$ does not intersect the support of $\chi_{c}$, that is, $\chi_{c} \chi_{p} \equiv 0$. Moreover, $\chi_{p} \equiv 1$ in a neighborhood of the poles $\mathcal{Y}_{p}$.

6.3.3. Derivation of the desired estimate. The rest of the derivation of estimate (6.26) is almost the same as the problem dealt with in [11, Section 5.5-5.8]. We shall follow the argument in [11] and only include the basic estimates for different components of $W^{n c}$. We refer the reader to [11] for the detailed proof. For the estimate of $T_{\chi_{c}}^{\lambda} W^{n c}$, we have

$$
\begin{aligned}
& \lambda\left(Z_{21, \lambda}^{-2}+Z_{21, \lambda}^{+2}+T_{\sigma_{l}}^{\lambda} Z_{10}^{-2}+T_{\sigma_{l}}^{\lambda} Z_{10}^{+2}\right) \\
& \quad+\lambda^{3}\left(Z_{10}^{-2}+\lambda^{2} Z_{10}^{+2}\right)+\left(\left\|Z_{2}^{-}(0)\right\|_{1, \lambda}^{2}+\left\|Z_{2}^{+}(0)\right\|_{1, \lambda}^{2}\right) \\
& \quad+\left(\left\|T_{\sigma}^{\lambda} Z_{1}^{-}(0)\right\|_{0}^{2}+\left\|T_{\sigma}^{\gamma} Z_{1}^{+}(0)\right\|_{0}^{2}\right)+\lambda^{2}\left(\left\|Z_{1}^{-}(0)\right\|_{0}^{2}+\left\|Z_{1}^{+}(0)\right\|_{0}^{2}\right) \\
& \leq \frac{C}{\lambda}\left(F_{1, \lambda}^{2}+W_{0}^{2}+T_{r}^{\lambda} W_{1, \lambda}^{2}\right)+\|G\|_{1, \lambda}+\left\|\left.W^{n c}\right|_{x_{2}=0}\right\|_{0}^{2},
\end{aligned}
$$

where the vectors $Z^{ \pm}$are defined as

$$
Z^{+}:=T_{\chi_{1}\left(Q_{0}^{r}+Q_{-1}^{r}\right)} T_{\chi_{c}}^{\lambda}\left(\begin{array}{l}
W_{3}^{+} \\
W_{4}^{+}
\end{array}\right), \quad Z^{-}:=T_{\chi_{1}\left(Q_{0}^{l}+Q_{-1}^{l}\right)} T_{\chi_{c}}^{\lambda}\left(\begin{array}{l}
W_{3}^{-} \\
W_{4}^{-}
\end{array}\right)
$$

and $\chi_{1}$ is a cut-off function satisfying $\chi_{1} \chi_{c} \equiv \chi_{c}$. The matrices $Q_{0}^{r, l}$ are invertible in a neighborhood of the support of $\chi_{1}$, and $r$ is a symbol in the class $\Gamma_{0}^{1}$ that vanishes in a neighborhood of the bicharacteristic curves. For the estimate of $T_{\chi_{u}}^{\lambda} W^{n c}$, we have

$$
\begin{aligned}
& \lambda T_{\chi_{u}}^{\lambda} W_{1, \lambda}^{2}+\left\|T_{\chi_{u}}^{\lambda} W^{n c}(0)\right\|_{1, \lambda}^{2} \\
\leq & C\left(\|G\|_{1, \lambda}^{2}+\left\|W^{n c}(0)\right\|_{0}^{2}\right)+\frac{C}{\lambda}\left(F_{1, \lambda}^{2}+W_{0}^{2}+T_{r}^{\lambda} W_{1, \lambda}^{2}\right) .
\end{aligned}
$$

Finally, the estimate of $T_{\chi_{p}}^{\lambda} W^{n c}$ says

$$
\begin{aligned}
& \lambda T_{\chi_{p}}^{\lambda} W_{1, \lambda}^{2}+\left\|T_{\chi_{p}}^{\lambda} W^{n c}(0)\right\|_{1, \lambda}^{2} \\
\leq & C\left(\|G\|_{1, \lambda}^{2}+\left\|W^{n c}(0)\right\|_{0}^{2}\right)+\frac{C}{\lambda}\left(F_{1, \lambda}^{2}+W_{0}^{2}+T_{r}^{\lambda} W_{1, \lambda}^{2}\right) .
\end{aligned}
$$

Combining (6.46), (6.47), and (6.48), then using the technique developed in [11] to get rid of the term $T_{r}^{\lambda} W_{1, \lambda}$, we finished the proof of (6.26).

6.4. The proof of Theorem 5.1. With the above estimates in hand, the proof of Theorem 5.1 is straightforward as follows. We first write

$$
\begin{aligned}
& T_{\tau}^{\lambda} \mathbf{A}_{0}^{r}+i \eta \mathbf{A}_{1}^{r}+\mathbf{A}_{0}^{r} \mathbf{C}^{r} W^{+}+\mathbf{I}_{2} \partial_{2} W^{+}=\mathcal{L}_{r}^{\lambda} W^{+}+\text {error }, \\
& T_{\tau}^{\lambda} \mathbf{A}_{0}^{l}+i \eta \mathbf{A}_{1}^{l}+\mathbf{A}_{0}^{l} \mathbf{C}^{l} W^{-}+\mathbf{I}_{2} \partial_{2} W^{-}=\mathcal{L}_{l}^{\lambda} W^{-}+\text {error }
\end{aligned}
$$


and estimate the error terms with the help of (6.19) and (6.20). We use (6.19), (6.20), (6.26), and (6.49) to derive

$$
\begin{aligned}
\left\|\left.W^{n c}\right|_{x_{2}=0}\right\|_{0}^{2} & \leq C_{0}\left(\left.\frac{1}{\lambda^{3}}\|\tilde{F}\|\right|_{1, \lambda} ^{2}+\frac{1}{\lambda^{2}}\left\||\tilde{G} \||_{1, \lambda}^{2}\right)\right. \\
& \leq C_{0}\left(\left.\frac{1}{\lambda^{3}}\left\|\left|\mathcal{L}^{\lambda} W\left\|\left.\right|_{1, \lambda} ^{2}+\frac{1}{\lambda^{3}}\right\|\right| \text { error }\right\|\right|_{1, \lambda} ^{2}+\frac{1}{\lambda^{2}}\left\||\tilde{G} \||_{1, \lambda}^{2}\right)\right. \\
& \leq C_{0}^{\prime}\left(\left.\frac{1}{\lambda^{3}}\left\|\left|\mathcal{L}^{\lambda} W\left\|\left.\right|_{1, \lambda} ^{2}+\frac{1}{\lambda^{3}}\right\|\right| W\right\|\right|_{0} ^{2}+\left.\frac{1}{\lambda^{2}}\left\||\tilde{G}|_{x_{2}=0}\right\|\right|_{1, \lambda} ^{2}\right) \\
& \leq C_{0}^{\prime}\left(\left.\frac{1}{\lambda^{3}}\left\|\left|\mathcal{L}^{\lambda} W\left\|\left.\right|_{1, \lambda} ^{2}+\frac{1}{\lambda^{3}}\right\|\right| W\right\|\right|_{0} ^{2}+\left.\frac{1}{\lambda^{2}}\left\|\left|T_{\beta}^{\lambda} W\right|_{x_{2}=0}\right\|\right|_{1, \lambda} ^{2}\right),
\end{aligned}
$$

where, as usual, $\mathcal{L}^{\lambda} W=\left(\mathcal{L}_{r}^{\lambda} W^{+}, \mathcal{L}_{l}^{\lambda} W^{-}\right)$. Using (6.21) and (6.23), and choosing $\lambda$ large enough, we obtain the following inequality:

$$
\begin{aligned}
& \left\|\left.W^{n c}\right|_{x_{2}=0}\right\|_{0}^{2}+\|\psi\|_{1, \lambda}^{2} \\
\leq & C_{0}^{\prime \prime}\left(\left.\frac{1}{\lambda^{3}}\left\|\left|\mathcal{L}^{\lambda} W\left\|\left.\right|_{1, \lambda} ^{2}+\frac{1}{\lambda^{3}}\right\|\right| W\right\|\right|_{0} ^{2}+\frac{1}{\lambda^{2}}\left\|T_{\mathbf{b}}^{\lambda} \psi+\left.T_{\mathbf{M}}^{\lambda} W\right|_{x_{2}=0}\right\|_{1, \lambda}^{2}\right) .
\end{aligned}
$$

Finally, we use (6.18) to derive (choosing $\lambda$ large enough)

$$
\begin{aligned}
& \left\|\left.W^{n c}\right|_{x_{2}=0}\right\|_{0}^{2}+\|\psi\|_{1, \lambda}^{2} \\
\leq & C_{0}^{\prime \prime \prime}\left(\frac { 1 } { \lambda ^ { 3 } } \left\|\left|\mathcal{L}^{\lambda} W\left\|\left.\right|_{1, \lambda} ^{2}+\frac{1}{\lambda^{3}}\right\| W\left\|\left.\right|_{0} ^{2}+\frac{1}{\lambda^{2}}\right\| \mathcal{B}^{\lambda}(W, \psi) \|_{1, \lambda}^{2}\right) .\right.\right.
\end{aligned}
$$

Then, one uses the definitions

$$
\begin{aligned}
& \mathrm{e}^{-\lambda t} \dot{V}_{+}=T_{r} W^{+}, \quad \mathrm{e}^{-\lambda t} \dot{V}_{-}=T_{l} W^{-}, \\
& \mathrm{e}^{-\lambda t} \mathbf{A}_{0}^{r} T_{r}^{-1} L_{r}^{\prime} \dot{V}_{+}=\mathcal{L}_{r}^{\lambda} W^{+}, \quad \mathrm{e}^{-\lambda t} \mathbf{A}_{0}^{l} T_{l}^{-1} L_{l}^{\prime} \dot{V}_{-}=\mathcal{L}_{l}^{\lambda} W^{-},
\end{aligned}
$$

as well as (5.17) and Lemma 5.2 to derive (5.12). One can easily check that the constants $C_{0}^{\prime}, C_{0}^{\prime \prime}$ and so on involved in the energy estimates only depend on $K_{0}$ and $\kappa_{0}$. This completes the proof of Theorem 5.1.

Appendix A. Derivation of Equations (6.5). This appendix gives the details of derivation of the equations (6.5). Multiplying the left side of the first equation in (5.11) by $A_{0}^{r} T_{r}^{-1}$, we obtain

$$
\begin{aligned}
& A_{0}^{r} T_{r}^{-1}\left\{L\left(U_{r}, \Phi_{r}\right) \dot{V}_{+}+C\left(U_{r}, \nabla U_{r}, \nabla \Phi_{r}\right) \dot{V}_{+}\right\} \\
= & A_{0}^{r} T_{r}^{-1} \partial_{t} \dot{V}_{+}+A_{0}^{r} T_{r}^{-1} A_{1}\left(U_{r}\right) \partial_{1} \dot{V}_{+} \\
& +A_{0}^{r} T_{r}^{-1} \frac{1}{\partial_{2} \Phi_{r}}\left[A_{2}\left(U_{r}\right)-\partial_{t} \Phi_{r} I_{4 \times 4}-\partial_{1} \Phi_{r} A_{1}\left(U_{r}\right)\right] \partial_{2} \dot{V}_{+}+A_{0}^{r} T_{r}^{-1} C\left(U_{r}, \nabla U_{r}, \nabla \Phi_{r}\right) \dot{V}_{+} \\
= & \sum_{i=1}^{4} R^{i},
\end{aligned}
$$

where

$$
\begin{aligned}
R^{1} & =A_{0}^{r} \partial_{t}\left(T_{r}^{-1} \dot{V}_{+}\right)-A_{0}^{r}\left(\partial_{t} T_{r}^{-1}\right) \dot{V}_{+}=A_{0}^{r} \partial_{t} W^{+}-A_{0}^{r}\left(\partial_{t} T_{r}^{-1}\right) T_{r} W^{+} \\
& =A_{0}^{r} \partial_{t} W^{+}-A_{0}^{r}\left[\partial_{t}\left(T_{r}^{-1} T_{r}\right)\right] W^{+}+A_{0}^{r} T_{r}^{-1}\left(\partial_{t} T_{r}\right) W^{+} \\
& =A_{0}^{r} \partial_{t} W^{+}+A_{0}^{r} T_{r}^{-1}\left(\partial_{t} T_{r}\right) W^{+}
\end{aligned}
$$




$$
\begin{aligned}
R^{2} & =\left[A_{0}^{r} T_{r}^{-1} A_{1}\left(U_{r}\right) T_{r}\right]\left[T_{r}^{-1} \partial_{1} \dot{V}_{+}\right]=A_{1}^{r}\left[T_{r}^{-1} \partial_{1} \dot{V}_{+}\right] \\
& =A_{1}^{r}\left[\partial_{1}\left(T_{r}^{-1} \dot{V}_{+}\right)\right]-A_{1}^{r}\left[\left(\partial_{1} T_{r}^{-1}\right) \dot{V}_{+}\right] \\
& =A_{1}^{r} \partial_{1} W^{+}-A_{1}^{r}\left[\left(\partial_{1} T_{r}^{-1}\right) T_{r}\right]\left[T_{r}^{-1} \dot{V}_{+}\right] \\
& =A_{1}^{r} \partial_{1} W^{+}-A_{1}^{r}\left[\partial_{1}\left(T_{r}^{-1} T_{r}\right)\right] W^{+}+A_{1}^{r} T_{r}^{-1}\left(\partial_{1} T_{r}\right) W^{+} \\
& =A_{1}^{r} \partial_{1} W^{+}+A_{1}^{r} T_{r}^{-1}\left(\partial_{1} T_{r}\right) W^{+} \\
& =A_{1}^{r} \partial_{1} W^{+}+\left(A_{0}^{r} T_{r}^{-1} A_{1} T_{r}\right) T_{r}^{-1}\left(\partial_{1} T_{r}\right) W^{+} \\
& =A_{1}^{r} \partial_{1} W^{+}+A_{0}^{r} T_{r}^{-1} A_{1}\left(\partial_{1} T_{r}\right) W^{+}, \\
R^{3} & =A_{0}^{r} T_{r}^{-1} \bar{A}_{2} \partial_{2} \dot{V}_{+}=\left(A_{0}^{r} T_{r}^{-1} \bar{A}_{2} T_{r}\right)\left(T_{r}^{-1} \partial_{2} \dot{V}_{+}\right)=I_{2}\left(T_{r}^{-1} \partial_{2} \dot{V}_{+}\right) \\
& =I_{2}\left[\partial_{2}\left(T_{r}^{-1} \dot{V}_{+}\right)\right]-I_{2}\left[\left(\partial_{2} T_{r}^{-1}\right) \dot{V}_{+}\right] \\
& =I_{2} \partial_{2} W^{+}-I_{2}\left[\left(\partial_{2} T_{r}^{-1}\right) T_{r}\right]\left[T_{r}^{-1} \dot{V}_{+}\right] \\
& =I_{2} \partial_{2} W^{+}-I_{2}\left[\partial_{2}\left(T_{r}^{-1} T_{r}\right)\right] W^{+}+I_{2} T_{r}^{-1}\left(\partial_{2} T_{r}\right) W^{+} \\
& =I_{2} \partial_{2} W^{+}+I_{2} T_{r}^{-1}\left(\partial_{2} T_{r}\right) W^{+} \\
& =I_{2} \partial_{2} W^{+}+\left(A_{0}^{r} T_{r}^{-1} \bar{A}_{2} T_{r}\right) T_{r}^{-1}\left(\partial_{2} T_{r}\right) W^{+} \\
& =I_{2} \partial_{2} W^{+}+A_{0}^{r} T_{r}^{-1} \bar{A}_{2}\left(\partial_{2} T_{r}\right) W^{+},
\end{aligned}
$$

and

$$
R^{4}=\left(A_{0}^{r} T_{r}^{-1} C T_{r}\right)\left(T_{r}^{-1} \dot{V}_{+}\right)=\left(A_{0}^{r} T_{r}^{-1} C T_{r}\right) W^{+}
$$

Thus,

$$
\begin{aligned}
\sum_{i=1}^{4} R^{i}= & A_{0}^{r} \partial_{t} W^{+}+A_{0}^{r} T_{r}^{-1}\left(\partial_{t} T_{r}\right) W^{+}+A_{1}^{r} \partial_{1} W^{+}+A_{0}^{r} T_{r}^{-1} A_{1}\left(\partial_{1} T_{r}\right) W^{+} \\
& +I_{2} \partial_{2} W^{+}+A_{0}^{r} T_{r}^{-1} \bar{A}_{2}\left(\partial_{2} T_{r}\right) W^{+}+\left(A_{0}^{r} T_{r}^{-1} C T_{r}\right) W^{+} \\
= & A_{0}^{r} \partial_{t} W^{+}+A_{1}^{r} \partial_{1} W^{+}+I_{2} \partial_{2} W^{+} \\
& +A_{0}^{r}\left[T_{r}^{-1}\left(\partial_{t} T_{r}\right)+T_{r}^{-1} A_{1}\left(\partial_{1} T_{r}\right)+T_{r}^{-1} \bar{A}_{2}\left(\partial_{2} T_{r}\right)+T_{r}^{-1} C T_{r}\right] W^{+}
\end{aligned}
$$

which yields the first equation in $(6.5)$ on $W^{+}$. The second equation in $(6.5)$ on $W^{-}$ can be obtained similarly.

Acknowledgements. The research of L. Ruan and C. Zhu was supported in part by the Natural Science Foundation of China No. 11331005, Program for Changjiang Scholars and Innovative Research Team in University No. IRT13066, and the Special Fund for Basic Scientific Research of Central Colleges No. CCNU12C01001 and No. CCNU14G01005. D. Wang's research was supported in part by the National Science Foundation under Grant DMS-1312800.

\section{REFERENCES}

[1] S. Alinhac, Existence of rarefaction waves for multidimensional hyperbolic quasilinear systems, Commun. Part. Diff. Eqs., 14, 173-230, 1989. 
[2] D.M. Ambrose and N. Masmoudi, Well-posedness of $3 D$ vortex sheets with surface tension, Commun. Math. Sci., 5, 391-430, 2007.

[3] M. Baudin, C. Berthon, F. Coquel, R. Masson, and Q. H. Tran, A relaxation method for two-phase flow models with hydrodynamic closure law, Numer. Math., 99, 411-440, 2005.

[4] M. Baudin, F. Coquel, and Q. H. Tran, A semi-implicit relaxation scheme for modeling two-phase flow in a pipeline, SIAM J. Sci. Comput., 27, 914-936, 2005.

[5] J. Bony, Symbolic calculus and propagation of singularities for nonlinear partial differential equations, Ann. Sci. Ecole Norm. Sup., 14, 209-246, 1981.

[6] C.E. Brennen, Fundamentals of Multiphase Flow, Cambridge University Press, New York, 2005.

[7] J.Y. Chemin, Perfect Imcompressible Fluids, Oxford University Press, 1998.

[8] G.-Q. Chen and Y.-G. Wang, Existence and stability of compressible current-vortex sheets in threedimensional magnetohydrodynamics, Arch. Ration. Mech. Anal., 187, 369-408, 2008.

[9] J. Cortes, A. Debussche, and I. Toumi, A density perturbation method to study the eigenstructure of two-phase flow equation systems, J. Comput. Phys., 147, 463-484, 1998.

[10] J.F. Coulombel and A. Morando, Stability of contact discontinuities for the nonisentropic Euler equations, Ann. Univ. Ferrara Sez (N.S.), 50, 79-90, 2004.

[11] J.F. Coulombel and P. Secchi, The stability of compressible vortex sheets in two space dimensions, Indiana Univ. Math. J., 53, 941-1012, 2004.

[12] J.F. Coulombel and P. Secchi, Nonlinear compressible vortex sheets in two space dimensions, Ann. Sci. Ec. Norm. Super., 41, 85-139, 2008.

[13] S. Evje, Global weak solutions for a compressible gas-liquid model with well-formation interaction, J. Diff. Eqs., 251, 2352-2386, 2011.

[14] S. Evje, Weak solutions for a gas-liquid model relevant for describing gas-kick in oil wells, SIAM J. Math. Anal., 43, 1887-1922, 2011.

[15] S. Evje and K.K. Fjelde, Relaxation schemes for a two-phase flow model, Math. Comput. Modelling, 36, 535-567, 2002.

[16] S. Evje and K.K. Fjelde, On a rough AUSM scheme for a one dimensional two-phase model, Comput. \& Fluids, 32, 1497-1530, 2003.

[17] S. Evje and T. Flatten, On the wave structure of two-phase model, SIAM J. Appl. Math., 67, 487-511, 2007.

[18] S. Evje and K.H. Karlsen, Global existence of weak solutions for a viscous two-phase model, J. Diff. Eqs., 245, 2660-2703, 2008.

[19] S. Evje and K.H. Karlsen, Global weak solutions for a viscous liquid-gas model with singular pressure law, Commun. Pure Appl. Anal., 8, 1867-1894, 2009.

[20] J.A. Fejer and W. Miles, On the stability of a plane vortex sheet with respect to three dimensional disturbances, J. Fluid Mech., 15, 335-336, 1963.

[21] T. Flatten and S.T. Munkejord, The approximate Riemann solver of Roe applied to a drift-flux two-phase flow model, ESAIM: Math. Mod. Num. Anal., 40, 735-764, 2006.

[22] J. Francheteau and G. Metivier, Existence of weak shocks for multidimensional hyperbolic quasilinear systems, Asterisque, 268, 1-198, 2000.

[23] H.A. Friis and S. Evje, Global weak solutions for a gas-liquid model with external forces and general pressure law, SIAM J. Appl. Math., 71, 409-442, 2011.

[24] H.A. Friis and S. Evje, Well-posedness of a compressible gas-liquid model with a friction term important for well control operations, SIAM J. Appl. Math., 71, 2014-2047, 2011.

[25] S.B. Gavage and D. Serre, First order systems of hyperbolic partial differential equations with applications, The Clarendon Press, Oxford University Press, Oxford, 2007.

[26] C.-C. Hao and H.-L. Li, Well-posedness for a multidimensional viscous liquid-gas two-phase flow model, SIAM J. Math. Anal., 44, 1304-1332, 2012.

[27] R. Hersh, Mixed problems in several variables, J. Math. Mech., 12, 317-334, 1963.

[28] M. Ishii and T. Hibiki, Thermo-fluid Dynamics of Two-Phase Flow, Springer-Verlag, New York, 2006 .

[29] Q.-S. Jiu and Z.-P. Xin, On strong convergence to 3-D axisymmetric vortex sheets, J. Diff. Eqs., 223, 33-50, 2006.

[30] Q.-S. Jiu and Z.-P. Xin, On strong convergence to 3D steady vortex sheets, J. Diff. Eqs., 239, 448-470, 2007.

[31] N.I. Kolev, Multiphase Flow Dynamics. Vol. 1. Fundamentals, Springer-Verlag, Berlin, 2005.

[32] N.I. Kolev, Multiphase Flow Dynamics. Vol. 2. Thermal and Mechanical Interactions, SpringerVerlag, Berlin, 2005.

[33] P.D. Lax, Hyperbolic systems of conservation laws. II, Commun. Pure Appl. Math., 10, 537-566, 1957.

[34] P.D. Lax and R.S. Phillips, Local boundary conditions for dissipative symmetric linear differential operators, Commun. Pure Appl. Math., 13, 427-455, 1960. 
[35] Q.-Q. Liu and C.-J. Zhu, Asymptotic behavior of a viscous liquid-gas model with mass-dependent viscosity and vacuum, J. Diff. Eqs., 252, 2492-2519, 2012.

[36] M.C. Lopes Filho, H.J. Nussenzveig Lopes, and Z.-P. Xin, Existence of vortex sheets with reflection symmetry in two space dimensions, Arch. Ration. Mech. Anal., 158, 235-257, 2001.

[37] M.-C. Lopes Filho, H.-J. Nussenzveig Lopes, and Z.-P. Xin, Existence of vortex sheets with reflection symmetry in two space dimensions, J. Diff. Eqs., 229, 154-171, 2006.

[38] A. Majda and S. Osher, Initial-boundary value problems for hyperbolic equations with uniformly characteristic boundary, Commun. Pure Appl. Math., 28, 607-675, 1975.

[39] A. Majda, The stability of multidimensional shock fronts, Mem. Amer. Math. Soc., 41, 1983.

[40] A. Majda, The existence of multidimensional shock fronts, Mem. Amer. Math. Soc., 43, 1983.

[41] A. Majda and A.L. Bertozzi, Vorticity and incompressible flow, Cambridge Texts in Applied Mathematics, Cambridge University Press, Cambridge, 27, 2002.

[42] Y. Meyer, Remarks on a theorem of J.-M. Bony, Rend. Circ. Mat. Palermo, 2, 1-20, 1981.

[43] J.W. Miles, On the disturbed motion of a plane vortex sheet, J. Fluid Mech., 4, 538-552, 1958.

[44] A. Morando and P. Trebeschi, Two-dimensional vortex sheets for the nonisentropic Euler equations: linear stability, J. Hyperbolic Differ. Equ., 5, 487-518, 2008.

[45] D. Serre, Systems of Conservation Laws. 1: Hyperbolicity, Entropies, Shock Wave, Cambridge University Press, Cambridge, 1999.

[46] D. Serre, Systems of Conservation Laws. 2: Geometric structures, oscillations, and initialboundary value problems, Cambridge University Press, Cambridge, 2000.

[47] C. Sulem, P. L. Sulem, C. Bardos, and U. Frisch, Finite time analyticity for the two- and threedimensional Kelvin-Helmholtz instability, Comm. Math. Phys., 80, 485-516, 1981.

[48] Y. Trakhinin, Existence of compressible current-vortex sheets: variable coefficients linear analysis, Arch. Ration. Mech. Anal., 177, 331-366, 2005.

[49] C. Wang and Z.-F. Zhang, A new proof of Wu's Theorem on vortex sheets, Sci. China Math., 55, 1449-1462, 2012.

[50] Y.-G. Wang and F. Yu, Stability of contact discontinuities in three-dimensional compressible steady flows, J. Diff. Eqs., 255, 1278-1356, 2013.

[51] Y.-G. Wang and F. Yu, Stabilization effect of magnetic fields on two-dimensional compressible current-vortex sheets, Arch. Ration. Mech. Anal., 208, 341-389, 2013.

[52] Y.-G. Wang and F. Yu, Structural stability of supersonic contact discontinuities in threedimensional compressible steady flows, preprint arXiv:1407.1464, (2014).

[53] H.-Y. Wen, L. Yao, and C.-J. Zhu, A blow-up criterion of strong solution to a $3 D$ viscous liquid-gas two-phase flow model with vacuum, J. Math. Pures Appl., 97, 204-229, 2012.

[54] S. Wu, Mathematical analysis of vortex sheets, Commun. Pure Appl. Math., 59, 1065-1206, 2006.

[55] L. Yao, T. Zhang, and C.-J. Zhu, Existence of asymptotic behavior of global weak solutions to a $2 D$ viscous liquid-gas two-phase flow model, SIAM J. Math. Anal., 42, 1874-1897, 2010.

[56] L. Yao, T. Zhang, and C.-J. Zhu, A blow-up criterion for a $2 D$ viscous liquid-gas two-phase flow model, J. Diff. Eqs., 250, 3362-3378, 2011.

[57] L. Yao and C.-J. Zhu, Free boundary value problem for a viscous two-phase model with massdependent viscosity, J. Diff. Eqs., 247, 2705-2739, 2009.

[58] L. Yao and C.-J. Zhu, Existence and uniqueness of global weak solution to a two-phase flow model with vacuum, Math. Ann., 349, 903-928, 2011. 